Kyushu J. Math.

Vol. 59, 2005, pp. 155-230

\title{
ON THE HYPOELLIPTICITY WITH A BIG LOSS OF DERIVATIVES
}

\author{
Cesare PARENTI and Alberto PARMEGGIANI
}

(Received 9 April 2004)

\begin{abstract}
We study, for a model class of classical pseudodifferential operators with symplectic characteristics of multiplicity $k$, necessary and sufficient conditions for the hypoellipticity with loss of $r+k / 2$ derivatives $(r>0)$.
\end{abstract}

\section{Introduction and setting of the problem}

Consider, for $n \geq 2$ and $1 \leq v<n$,

$$
T^{*} \mathbb{R}^{n}=\mathbb{R}_{z}^{n} \times \mathbb{R}_{\zeta}^{n}=\left(\mathbb{R}_{x}^{v} \times \mathbb{R}_{y}^{n-v}\right) \times\left(\mathbb{R}_{\xi}^{v} \times \mathbb{R}_{\eta}^{n-v}\right)
$$

and define

$$
\Sigma=\left\{(z, \zeta) \in T^{*} \mathbb{R}^{n} \backslash 0 ; x=\xi=0\right\} .
$$

Suppose we are given in $\mathbb{R}^{n}$ a (properly supported) pseudodifferential operator $(\psi$ do) $A=A\left(z, D_{z}\right)$ of order $m \in \mathbb{R}, A \in \mathrm{OPS}_{\mathrm{cl}}^{m}\left(\mathbb{R}^{n}\right)$. By this we mean that the symbol $a(z, \zeta)$ admits an asymptotic semi-regular expansion of the kind

$$
a(z, \zeta) \sim \sum_{j \geq 0} a_{m-j / 2}(z, \zeta)
$$

where $a_{m-j / 2}$ is smooth (i.e. $C^{\infty}$ ) on $T^{*} \mathbb{R}^{n} \backslash 0$ and (positively) homogeneous of degree $m-j / 2$ in the fiber variable $\zeta$. Suppose, furthermore, that for a given integer $k \geq 1$, we have

$$
\begin{gathered}
\left|a_{m-j / 2}(z, \zeta)\right| \lesssim|\zeta|^{m-j / 2} \operatorname{dist}_{\Sigma}(z, \zeta)^{k-j} \quad j=0, \ldots, k \\
|\zeta|^{m} \operatorname{dist}_{\Sigma}(z, \zeta)^{k} \lesssim\left|a_{m}(z, \zeta)\right|
\end{gathered}
$$

2000 Mathematics Subject Classification: Primary 35H10;

$$
\text { Secondary 35S99. }
$$

Keywords and Phrases: hypoellipticity with loss of derivatives; transversal ellipticity; parametrix. 
where $\operatorname{dist}_{\Sigma}(z=(x, y), \zeta=(\xi, \eta))=|x|+|\xi| /|\zeta|$ denotes the distance of $(z, \zeta /|\zeta|)$ to $\Sigma$, and the notation $f \lesssim g$ means that, for any given conic set $\Gamma \subset T^{*} \mathbb{R}^{n} \backslash 0$ with compact base and any given $\varepsilon>0$, there exists $C=C_{\Gamma, \varepsilon}>0$ such that

$$
f(z, \zeta) \leq C g(z, \zeta) \quad \text { for all }(z, \zeta) \in \Gamma, \quad|\zeta| \geq \varepsilon .
$$

As usual, we rephrase condition (1) by saying that $A$ vanishes to order $k$ at $\Sigma$ and condition (2) by saying that $A$ is transversally elliptic (with respect to $\Sigma$ ).

We are interested in studying the hypoellipticity of $A$. More precisely, we shall stick to the following notions of hypoellipticity.

DEFINITION 1.1. We say that A is hypoelliptic at $\rho_{0} \in T^{*} \mathbb{R}^{n} \backslash 0$ with loss of $r \geq 0$ derivatives, respectively hypoelliptic at $\rho_{0}$, if for every distribution $u \in \mathcal{D}^{\prime}\left(\mathbb{R}^{n}\right)$ and any given $s \in \mathbb{R}$, we have

$$
A u \in H^{s} \quad \text { at } \rho_{0} \Longrightarrow u \in H^{s+m-r} \quad \text { at } \rho_{0},
$$

respectively for all $u \in \mathcal{D}^{\prime}\left(\mathbb{R}^{n}\right)$

$$
\rho_{0} \notin \mathrm{WF}(A u) \Longrightarrow \rho_{0} \notin \mathrm{WF}(u) .
$$

Furthermore, we say that $A$ is hypoelliptic at $z_{0} \in \mathbb{R}^{n}$ with loss of $r \geq 0$ derivatives, respectively hypoelliptic at $z_{0}$, if $A$ is hypoelliptic with loss of $r$ derivatives, respectively hypoelliptic, at $\rho_{0}=\left(z_{0}, \zeta\right)$ for all $\zeta \neq 0$.

REMARK 1.2. Recall that a distribution $v \in \mathcal{D}^{\prime}\left(\mathbb{R}^{n}\right)$ belongs to the Sobolev space $H^{t}$ at $\rho_{0}$ if and only if there exists $v^{\prime} \in H_{\mathrm{loc}}^{t}\left(\mathbb{R}^{n}\right)$ such that $\rho_{0} \notin \mathrm{WF}\left(v-v^{\prime}\right)$.

Equivalently, with $\rho_{0}=\left(z_{0}, \zeta_{0}\right)$, there exist a neighborhood $U$ of $z_{0}$ and a conic neighborhood $\Gamma$ of $\zeta_{0}$, such that

$$
\int_{\Gamma}\left(1+|\zeta|^{2}\right)^{t}|\widehat{\varphi v}(\zeta)|^{2} d \zeta<+\infty, \quad \text { for all } \varphi \in C_{0}^{\infty}(U) .
$$

When $\rho_{0} \notin \Sigma$, we have $a_{m}\left(\rho_{0}\right) \neq 0$ and by classical elliptic theory $A$ is hypoelliptic at $\rho_{0}$ with loss of zero derivatives. Thus, the problem is the hypoellipticity of $A$ at the points of $\Sigma$.

When $\rho_{0} \in \Sigma$, it is known (after Sjöstrand [19]) that $A$ cannot be hypoelliptic at $\rho_{0}$ with loss of $r<k / 2$ derivatives. The hypoellipticity with loss of $k / 2$ derivatives has been completely described by Boutet $e t a l$ in [4] (where they treat the general case of $\Sigma$ a closed and conic submanifold of $T^{*} \mathbb{R}^{n} \backslash 0$ ). 
The main tool for detecting hypoellipticity is the so called localized operator $A_{\rho}$ of $A$ at $\rho \in \Sigma$. In our case, for $\rho=(0, y, 0, \eta \neq 0), A_{\rho}$ can be defined as follows. Upon setting

$$
a_{\rho}(x, \xi):=\sum_{|\alpha|+|\beta|+j=k} \frac{1}{\alpha ! \beta !}\left(\partial_{x}^{\alpha} \partial_{\xi}^{\beta} a_{m-j / 2}\right)(\rho) x^{\alpha} \xi^{\beta},
$$

we define

$$
A_{\rho}=\operatorname{Op}\left(a_{\rho}\right)\left(x, D_{x}\right),
$$

where Op denotes the usual quantization in the $(x, \xi)$ variables.

REMARK 1.3. It is important to note that $A_{\rho}$ is a polynomial-coefficient differential operator of order $k$ in $\mathbb{R}^{v}$ which, by virtue of the transversal ellipticity, is globally elliptic in the sense of Shubin [18] (see also [8]). As a consequence, $A_{\rho}$ maps $\mathcal{S}\left(\mathbb{R}^{\nu}\right)$ (respectively $\mathcal{S}^{\prime}\left(\mathbb{R}^{\nu}\right)$ ) into itself continuously and

$$
f \in \mathcal{S}^{\prime}\left(\mathbb{R}^{\nu}\right), \quad A_{\rho} f \in \mathcal{S}\left(\mathbb{R}^{\nu}\right) \Longrightarrow f \in \mathcal{S}\left(\mathbb{R}^{\nu}\right) .
$$

Throughout the paper, we shall tacitly consider $A_{\rho}: L^{2}\left(\mathbb{R}^{v}\right) \longrightarrow L^{2}\left(\mathbb{R}^{v}\right)$ as an unbounded operator with domain

$$
B^{k}\left(\mathbb{R}^{\nu}\right):=\left\{f \in \mathcal{S}^{\prime}\left(\mathbb{R}^{\nu}\right) ;\|f\|_{B^{k}\left(\mathbb{R}^{\nu}\right)}:=\left(\sum_{|\alpha|+|\beta| \leq k}\left\|x^{\alpha} D_{x}^{\beta} f\right\|_{L^{2}\left(\mathbb{R}^{\nu}\right)}^{2}\right)^{1 / 2}<+\infty\right\} .
$$

Recall that $B^{k}\left(\mathbb{R}^{v}\right)$ is dense in $L^{2}\left(\mathbb{R}^{v}\right)$ with compact embedding.

The global ellipticity of $A_{\rho}$ means that $A_{\rho}$ has a finite dimensional kernel and a closed range with finite codimension (so that $A_{\rho}$ is a Fredholm operator). Moreover, $\operatorname{Spec}\left(A_{\rho}\right)$, the spectrum of $A_{\rho}$, is either the whole complex plane or a discrete set, made of a sequence of eigenvalues of finite multiplicities.

This latter case, via Shubin theory, occurs if the numerical range (i.e. the set of values) of the polynomial

$$
\tilde{a}_{\rho}(x, \xi):=\sum_{|\alpha|+|\beta|=k} \frac{1}{\alpha ! \beta !}\left(\partial_{x}^{\alpha} \partial_{\xi}^{\beta} a_{m}\right)(\rho) x^{\alpha} \xi^{\beta},
$$

which is a closed connected cone of $\mathbb{C}$, is not the whole complex plane $\mathbb{C}$.

The Boutet-Grigis-Helffer theorem reads as follows.

THEOREM 1.4. A is hypoelliptic at $\rho_{0} \in \Sigma$ with loss of $k / 2$ derivatives if and only if

$$
\operatorname{Ker}\left(A_{\rho_{0}}\right)=\{0\} .
$$


In this paper, we consider cases in which condition (7) is violated at some point $\rho_{0}$ of $\Sigma$, and our purpose is to give (inter alia) necessary and sufficient conditions for the hypoellipticity of $A$ with a loss of derivatives greater than $k / 2$.

More precisely, on supposing that $\operatorname{Ker}\left(A_{\rho_{0}}\right) \neq\{0\}$ for some $\rho_{0} \in \Sigma$ and that $A_{\rho}$ has a discrete spectrum for $\rho$ near $\rho_{0}$, we shall show that it is possible to microlocally associate with $A$ pseudodifferential square systems $\Lambda=\Lambda\left(y, D_{y}\right)$ in $\mathbb{R}^{n-v}$ of order $m-k / 2$ and size $\operatorname{dim} \operatorname{Ker}\left(A_{\rho_{0}}\right)$ (how $\Lambda$ is associated with $A$ is made precise in Definition 4.5 and Theorem 4.6) so that, after canonically identifying $\Sigma$ with $T^{*} \mathbb{R}^{n-v} \backslash 0$, the hypoellipticity of $A$ at $\rho_{0}$ with loss of $r+k / 2$ derivatives $(r>0)$ is equivalent to the hypoellipticity of each $\Lambda$ at $\rho_{0}$ with loss of $r$ derivatives. The complete symbol of every system $\Lambda$ will be explicitly constructed.

The very idea of associating the systems $\Lambda$ with $A$ goes back to the seminal work of Sjöstrand [19] and was further exploited by Helffer [7] in his study of hypoellipticity with loss of $3 / 2$ derivatives for operators with symplectic double characteristics (i.e. $k=2, r=1 / 2$ ), and by Grigis and Rothschild [5] in their study of the analytic (and $C^{\infty}$ ) hypoellipticity of a class of differential operators with polynomial coefficients. In this respect, the present work is a natural continuation of these papers.

We point out that a serious limitation of our approach is the lack of invariance (see Section 7). Because of this, in treating examples (see Section 6), we are forced to consider only Grushin-type operators. Nevertheless, the examples we analyze seem to be new and also unexpected (at least to us). In particular, in Example 6.5, we show an instance of a transversally elliptic operator with characteristics of order four that, depending upon the choice of the lower-order terms, is hypoelliptic with loss of two, three, four or five derivatives.

\section{Hypotheses on the localized operator $A_{\rho}$ and related constructions}

As mentioned in the Introduction, we suppose that $\operatorname{Ker}\left(A_{\rho_{0}}\right) \neq\{0\}$ for some $\rho_{0} \in \Sigma$ and make our main hypothesis (H1):

(H1). The localized operator

$$
A_{\rho}: L^{2}\left(\mathbb{R}^{v}\right) \longrightarrow L^{2}\left(\mathbb{R}^{v}\right), \quad \operatorname{Dom}\left(A_{\rho}\right)=B^{k}\left(\mathbb{R}^{v}\right)
$$

has a discrete spectrum for all $\rho \in \Sigma$.

In the following, we shall always identify $\Sigma$ with $T^{*} \mathbb{R}^{n-v} \backslash 0$. 
It is crucial to observe the following homogeneity property of $A_{\rho}$. Consider, for $t>0$, the dilations

$$
\left(\mathrm{M}_{t} f\right)(x):=t^{v / 4} f\left(t^{1 / 2} x\right)
$$

that are isometries of $L^{2}\left(\mathbb{R}^{v}\right)$ (and automorphisms of $\mathcal{S}\left(\mathbb{R}^{v}\right)$, respectively $\mathcal{S}^{\prime}\left(\mathbb{R}^{v}\right)$ ). Since, by (4),

$$
a_{(y, t \eta)}\left(t^{-1 / 2} x, t^{1 / 2} \xi\right)=t^{m-k / 2} a_{(y, \eta)}(x, \xi),
$$

we have

$$
A_{(y, t \eta)}\left(\mathrm{M}_{t} f\right)=t^{m-k / 2} \mathrm{M}_{t}\left(A_{(y, \eta)} f\right) .
$$

As a consequence,

$$
\mathrm{M}_{t}\left(\operatorname{Ker}\left(A_{(y, \eta)}\right)\right)=\operatorname{Ker}\left(A_{(y, t \eta)}\right), \quad \text { for all } t>0 .
$$

We now give a few consequences of hypothesis $(\mathrm{H} 1)$ that we gather in the next proposition.

\section{PROPOSITION 2.1.}

1) $\left(A^{*}\right)_{\rho}$ also has a discrete spectrum and $\operatorname{Spec}\left(\left(A^{*}\right)_{\rho}\right)=\overline{\operatorname{Spec}\left(A_{\rho}\right)}$, for all $\rho \in \Sigma$.

2) One has $\operatorname{ind}\left(A_{\rho}\right)=\operatorname{ind}\left(\left(A^{*}\right)_{\rho}\right)=0$ for all $\rho \in \Sigma$.

3) The order of vanishing $k$ is even.

Proof. Since the $\psi$ do $A^{*}$ (the formal adjoint of $A$ ) vanishes to order $k$ at $\Sigma$ and is transversally elliptic and since, as one can easily see, $\left(A^{*}\right)_{\rho}=A_{\rho}^{*}$ point 1$)$ is well known.

As regards point 2), we have that if $\lambda \in \mathbb{C} \backslash \operatorname{Spec}\left(A_{\rho}\right)$, then $A_{\rho}-\lambda: B^{k}\left(\mathbb{R}^{\nu}\right) \longrightarrow$ $L^{2}\left(\mathbb{R}^{v}\right)$ is an isomorphism, whence we obviously have ind $\left(A_{\rho}-\lambda\right)=0$. Now, $A_{\rho}=A_{\rho}-\lambda \mathrm{id}+\lambda \mathrm{id}$ and since $\mathrm{id}: B^{k}\left(\mathbb{R}^{v}\right) \longrightarrow L^{2}\left(\mathbb{R}^{v}\right)$ is compact, we get that $\operatorname{ind}\left(A_{\rho}\right)=\operatorname{ind}\left(A_{\rho}-\lambda\right)=0$. In particular, we have that

$$
\operatorname{dim} \operatorname{Ker}\left(A_{\rho}-\lambda\right)=\operatorname{dim} \operatorname{Ker}\left(A_{\rho}^{*}-\bar{\lambda}\right), \quad \text { for all } \lambda \in \mathbb{C}, \quad \text { for all } \rho \in \Sigma .
$$

As regards point 3), it is well known that when $v>1$, then $k$ must be even. When $v=1$, we immediately observe that $\operatorname{ind}\left(A_{\rho}\right)=\operatorname{ind}\left(\operatorname{Op}\left(\tilde{a}_{\rho}\right)\left(x, D_{x}\right)\right)$, where $\tilde{a}_{\rho}$ was defined in (6). In fact, $A_{\rho}-\operatorname{Op}\left(\tilde{a}_{\rho}\right)\left(x, D_{x}\right)$ is a global differential operator in $\mathbb{R}^{v}$ of order $k-1$ and, hence, it is a compact operator from $B^{k}\left(\mathbb{R}^{v}\right)$ into $L^{2}\left(\mathbb{R}^{v}\right)$. Now, if one puts

$$
k_{ \pm}:=\operatorname{card}\left\{\zeta \in \mathbb{C} ; \pm \operatorname{Im}(\zeta)>0, \tilde{a}_{\rho}(1, \zeta)=0\right\}
$$


we have

$$
\tilde{a}_{\rho}(x, \xi)=x^{k} \tilde{a}_{\rho}(1, \xi / x)=c \prod_{j=1}^{k_{+}}\left(\xi-\zeta_{j}^{+} x\right) \prod_{j=1}^{k_{-}}\left(\xi-\zeta_{j}^{-} x\right)
$$

where

$$
c=\frac{1}{k !}\left(\partial_{\xi}^{k} a_{m}\right)(\rho) \neq 0 \quad \text { and } \quad \tilde{a}_{\rho}\left(1, \zeta_{j}^{ \pm}\right)=0, \quad \pm \operatorname{Im}\left(\zeta_{j}^{ \pm}\right)>0
$$

As a consequence,

$$
0=\operatorname{ind}\left(\operatorname{Op}\left(\tilde{a}_{\rho}\right)\right)=\sum_{j=1}^{k_{+}} \operatorname{ind}\left(D_{x}-\zeta_{j}^{+} x\right)+\sum_{j=1}^{k_{-}} \operatorname{ind}\left(D_{x}-\zeta_{j}^{-} x\right)=k_{+}-k_{-},
$$

and since $k_{+}+k_{-}=k$ we have the statement.

REMARK 2.2. As a consequence of point 2 of Proposition 2.1, we have that if $\rho_{0} \in \Sigma$ with $\operatorname{Ker}\left(A_{\rho_{0}}\right) \neq\{0\}$, then also $\operatorname{Ker}\left(A_{\rho_{0}}^{*}\right) \neq\{0\}$, and the two spaces have the same dimension. (Because of this, the systems $\Lambda$ mentioned in the Introduction are square systems of size $\operatorname{dim} \operatorname{Ker}\left(A_{\rho_{0}}\right)$.)

As already noted (see Remark 1.3), as a consequence of Shubin's theory [18]

$$
\tilde{a}_{\rho}\left(T^{*} \mathbb{R}^{v}\right) \neq \mathbb{C} \Longrightarrow A_{\rho} \text { has a discrete spectrum. }
$$

After Sjöstrand [19], it is known that this condition is fulfilled when $k=2$ and $v>1$ and it is also fulfilled for $k=2$ and $v=1$ when $k_{+}=k_{-}\left(\tilde{a}_{\rho}\left(T^{*} \mathbb{R}^{v}\right)\right.$ is then a closed strictly convex cone of $\mathbb{C}$ ).

Now let $\rho_{0} \in \Sigma$ be a point where $\operatorname{Ker}\left(A_{\rho_{0}}\right) \neq\{0\}$. We may suppose, by (11), that $\rho_{0} \in \mathbb{S}^{*} \Sigma=\{(y, \eta) \in \Sigma ;|\eta|=1\}$. We set

$$
1 \leq d=\operatorname{dim} \operatorname{Ker}\left(A_{\rho_{0}}\right)=\operatorname{dim} \operatorname{Ker}\left(A_{\rho_{0}}^{*}\right) .
$$

Since $A_{\rho}^{*} A_{\rho}$ and $A_{\rho} A_{\rho}^{*}$ are globally elliptic, self-adjoint and non-negative differential operators of order $2 k$, with a discrete spectrum contained in $[0,+\infty)$, and since

$$
\operatorname{Ker}\left(A_{\rho}\right)=\operatorname{Ker}\left(A_{\rho}^{*} A_{\rho}\right), \quad \operatorname{Ker}\left(A_{\rho}^{*}\right)=\operatorname{Ker}\left(A_{\rho} A_{\rho}^{*}\right), \quad \text { for all } \rho \in \Sigma,
$$

it follows that

$$
0 \in \operatorname{Spec}\left(A_{\rho_{0}}^{*} A_{\rho_{0}}\right) \cap \operatorname{Spec}\left(A_{\rho_{0}} A_{\rho_{0}}^{*}\right)
$$

and

$$
\operatorname{dim} \operatorname{Ker}\left(A_{\rho_{0}}^{*} A_{\rho_{0}}\right)=\operatorname{dim} \operatorname{Ker}\left(A_{\rho_{0}} A_{\rho_{0}}^{*}\right)=d
$$


DefinItION 2.3. Let $\tilde{U} \subset \mathbb{S}^{*} \Sigma$ be a neighborhood of $\rho_{0} \in \mathbb{S}^{*} \Sigma$. We say that $\tilde{U}$ is admissible if for some $\varepsilon>0$, the following are fulfilled.

(i) $\varepsilon \notin \operatorname{Spec}\left(A_{\rho}^{*} A_{\rho}\right) \cup \operatorname{Spec}\left(A_{\rho} A_{\rho}^{*}\right)$, for all $\rho \in \tilde{U}$.

(ii) Upon setting

$$
\begin{aligned}
& V_{1}(\rho):=\bigoplus_{0 \leq \lambda<\varepsilon, \lambda \in \operatorname{Spec}\left(A_{\rho}^{*} A_{\rho}\right)} \operatorname{Ker}\left(A_{\rho}^{*} A_{\rho}-\lambda\right) \subset \mathcal{S}\left(\mathbb{R}^{v}\right), \\
& V_{2}(\rho):=\bigoplus_{0 \leq \lambda<\varepsilon, \lambda \in \operatorname{Spec}\left(A_{\rho} A_{\rho}^{*}\right)} \operatorname{Ker}\left(A_{\rho} A_{\rho}^{*}-\lambda\right) \subset \mathcal{S}\left(\mathbb{R}^{v}\right)
\end{aligned}
$$

$(\bigoplus$ is finite and orthogonal), we have

$$
\operatorname{dim} V_{1}(\rho)=\operatorname{dim} V_{2}(\rho)=d, \quad \text { for all } \rho \in \tilde{U} .
$$

(iii) The Hermitian (with respect to the $L^{2}\left(\mathbb{R}^{v}\right)$-inner product) vector-bundles of rank $d$,

$$
V_{j}:=\bigsqcup_{\rho \in \tilde{U}} V_{j}(\rho) \longrightarrow \tilde{U}, \quad j=1,2
$$

are trivial.

REMARK 2.4 .

1) The fact that $V_{1}$ and $V_{2}$ are indeed Hermitian vector-bundles of rank $d$ follows from the fact that $V_{j}(\rho)=\operatorname{Im}\left(\pi_{j}(\rho)\right), j=1,2$, where $\pi_{j}(\rho)$ are the orthogonal projectors defined by

$$
\begin{aligned}
& \pi_{1}(\rho)=\frac{1}{2 \pi i} \oint_{|\zeta|=\varepsilon}\left(\zeta-A_{\rho}^{*} A_{\rho}\right)^{-1} d \zeta, \\
& \pi_{2}(\rho)=\frac{1}{2 \pi i} \oint_{|\zeta|=\varepsilon}\left(\zeta-A_{\rho} A_{\rho}^{*}\right)^{-1} d \zeta, \quad \rho \in \tilde{U} .
\end{aligned}
$$

2) It is obvious that if conditions (i) and (ii) are satisfied, then upon possibly shrinking $\tilde{U}$ we may always suppose that (iii) also holds. Hence, the existence of an admissible neighborhood of $\rho_{0}$ is always granted.

We now list two fundamental consequences.

LEMMA 2.5. Let $\tilde{U}$ be admissible. One has

$$
A_{\rho}: V_{1}(\rho) \longrightarrow V_{2}(\rho), \quad A_{\rho}^{*}: V_{2}(\rho) \longrightarrow V_{1}(\rho), \quad \text { for all } \rho \in \tilde{U} .
$$


Proof. We start off by observing that

$$
A_{\rho}\left(\zeta-A_{\rho}^{*} A_{\rho}\right)=\left(\zeta-A_{\rho} A_{\rho}^{*}\right) A_{\rho}, \quad \text { for all } \zeta \in \mathbb{C}, \quad \text { for all } \rho \in \Sigma,
$$

so that

$$
\left(\zeta-A_{\rho} A_{\rho}^{*}\right)^{-1} A_{\rho}=A_{\rho}\left(\zeta-A_{\rho}^{*} A_{\rho}\right)^{-1}, \quad \text { for all } \rho \in \tilde{U},|\zeta|=\varepsilon,
$$

whence

$$
A_{\rho} \pi_{1}(\rho)=\pi_{2}(\rho) A_{\rho}, \quad A_{\rho}^{*} \pi_{2}(\rho)=\pi_{1}(\rho) A_{\rho}^{*}, \quad \text { for all } \rho \in \tilde{U}
$$

which concludes the proof.

REMARK 2.6. When $\rho=\rho_{0}, V_{1}\left(\rho_{0}\right)=\operatorname{Ker}\left(A_{\rho_{0}}\right)$, and $A_{\rho_{0}}\left(V_{1}\left(\rho_{0}\right)\right)=\{0\}$. Now let $\phi_{j}^{\prime}(\rho ;.) \in \mathcal{S}\left(\mathbb{R}^{v}\right), 1 \leq j \leq d$, and $\phi_{j}(\rho ;.) \in \mathcal{S}\left(\mathbb{R}^{v}\right), 1 \leq j \leq d$, respectively, be $L^{2}\left(\mathbb{R}^{v}\right)$-orthonormal and smooth in $\rho \in \tilde{U}$ bases of $V_{1}(\rho)$ and $V_{2}(\rho)$, respectively. Then the matrix representing $A_{\rho}: V_{1}(\rho) \longrightarrow V_{2}(\rho)$ in the bases $\phi^{\prime}, \phi$, is

$$
\Lambda(\rho)=\left(\Lambda_{\phi, \phi^{\prime}}^{(j r)}(\rho)\right)_{1 \leq j, r \leq d}=\left(\left(A_{\rho} \phi_{r}^{\prime}(\rho ; .), \phi_{j}(\rho ; .)\right)_{L^{2}\left(\mathbb{R}^{v}\right)}\right)_{1 \leq j, r \leq d} .
$$

This matrix is smooth in $\rho$ and vanishes at $\rho_{0}$.

Note that it is not necessary for the $\phi_{j}^{\prime}$ and $\phi_{j}$ to be eigenfunctions of $A_{\rho}^{*} A_{\rho}$ and $A_{\rho} A_{\rho}^{*}$, respectively.

LEMMA 2.7. Let $\tilde{U}$ be admissible and let $V_{j}(\rho)^{\perp}, \rho \in \tilde{U}$, be the $L^{2}\left(\mathbb{R}^{v}\right)$-orthogonal of $V_{j}(\rho), j=1,2$. Then

$$
A_{\rho}: B^{k}\left(\mathbb{R}^{v}\right) \cap V_{1}(\rho)^{\perp} \longrightarrow V_{2}(\rho)^{\perp} \quad \text { and } \quad A_{\rho}^{*}: B^{k}\left(\mathbb{R}^{\nu}\right) \cap V_{2}(\rho)^{\perp} \longrightarrow V_{1}(\rho)^{\perp},
$$

are isomorphisms for all $\rho \in \tilde{U}$.

Proof. We just prove the property for $A_{\rho}$, since the proof for $A_{\rho}^{*}$ is analogous. Let $f \in B^{k}\left(\mathbb{R}^{v}\right) \cap V_{1}(\rho)^{\perp}$ and let $g \in V_{2}(\rho)$. Hence, from Lemma 2.5, we get $A_{\rho}^{*} g \in V_{1}(\rho)$ and, hence,

$$
\left(A_{\rho} f, g\right)_{L^{2}\left(\mathbb{R}^{v}\right)}=\left(f, A_{\rho}^{*} g\right)_{L^{2}\left(\mathbb{R}^{v}\right)}=0 .
$$

Thus, $A_{\rho}\left(B^{k}\left(\mathbb{R}^{v}\right) \cap V_{1}(\rho)^{\perp}\right) \subset V_{2}(\rho)^{\perp}$ for all $\rho \in \tilde{U}$.

We now observe that

- $\quad B^{k}\left(\mathbb{R}^{v}\right) \cap V_{1}(\rho)^{\perp}$ is closed in $B^{k}\left(\mathbb{R}^{v}\right)$ (which is obvious);

- $\quad B^{k}\left(\mathbb{R}^{v}\right) \cap V_{1}(\rho)^{\perp}$ is $L^{2}\left(\mathbb{R}^{v}\right)$-orthogonal to $\operatorname{Ker}\left(A_{\rho}\right)$ (which follows from $\left.\operatorname{Ker}\left(A_{\rho}\right)=\operatorname{Ker}\left(A_{\rho}^{*} A_{\rho}\right) \subset V_{1}(\rho)\right)$. 
Now, because of the global ellipticity of $A_{\rho}, \rho \in \Sigma$, for $A_{\rho}$ we have the a priori estimate

$$
\|f\|_{B^{k}\left(\mathbb{R}^{v}\right)} \leq C\left(\left\|A_{\rho} f\right\|_{L^{2}\left(\mathbb{R}^{\nu}\right)}+\|f\|_{L^{2}\left(\mathbb{R}^{\nu}\right)}\right), \quad \text { for all } f \in B^{k}\left(\mathbb{R}^{\nu}\right) .
$$

As a consequence, we claim that if $T \subset B^{k}\left(\mathbb{R}^{\nu}\right)$ is closed in $B^{k}\left(\mathbb{R}^{\nu}\right)$ and $T \perp_{L^{2}}$ $\operatorname{Ker}\left(A_{\rho}\right)$, then $A_{\rho}(T)$ is closed in $L^{2}\left(\mathbb{R}^{v}\right)$. To see this, we need only prove that if $g \in \overline{A_{\rho}(T)}, g \neq 0$, then $g \in A_{\rho}(T)$. Hence, let $\left\{f_{j}\right\}_{j \geq 1} \subset T$ be a sequence such that $A_{\rho} f_{j} \stackrel{L^{2}}{\longrightarrow} g$. Then it must be

$$
\sup _{j \geq 1}\left\|f_{j}\right\|_{B^{k}\left(\mathbb{R}^{v}\right)}<+\infty
$$

Otherwise, by possibly passing to a subsequence, we would have $\left\|f_{j}\right\|_{B^{k}\left(\mathbb{R}^{v}\right)} \rightarrow$ $+\infty$ and, hence, $A_{\rho}\left(f_{j} /\left\|f_{j}\right\|_{B^{k}\left(\mathbb{R}^{\nu}\right)}\right) \stackrel{L^{2}}{\longrightarrow} 0$. Since $\left\|f_{j} /\right\| f_{j}\left\|_{B^{k}\left(\mathbb{R}^{\nu}\right)}\right\|_{B^{k}\left(\mathbb{R}^{v}\right)}=$ 1 , being $B^{k}\left(\mathbb{R}^{v}\right)$ compactly embedded in $L^{2}\left(\mathbb{R}^{v}\right)$, we have (again possibly for some subsequence) $f_{j} /\left\|f_{j}\right\|_{B^{k}\left(\mathbb{R}^{\nu}\right)} \stackrel{L^{2}}{\longrightarrow} \varphi \in L^{2}\left(\mathbb{R}^{\nu}\right)$, thereby yielding $A_{\rho}\left(f_{j} /\left\|f_{j}\right\|_{B^{k}\left(\mathbb{R}^{\nu}\right)} \stackrel{\mathcal{S}^{\prime}}{\longrightarrow} A_{\rho} \varphi\right.$ and, finally, that $A_{\rho} \varphi=0$, i.e. $\varphi \in \operatorname{Ker}\left(A_{\rho}\right)$. However, since, by hypothesis, $f_{j} /\left\|f_{j}\right\|_{B^{k}\left(\mathbb{R}^{\nu}\right)} \in T$ and $T \perp_{L^{2}} \operatorname{Ker}\left(A_{\rho}\right)$, we get ${ }_{B^{k}} \varphi \in \operatorname{Ker}\left(A_{\rho}\right)^{\perp}$ so that $\varphi=0$. But then, from (12), we obtain that $f_{j} /\left\|f_{j}\right\|_{B^{k}\left(\mathbb{R}^{v}\right)} \stackrel{B^{k}}{\longrightarrow} 0$, which is a contradiction. Hence,

$$
\sup _{j \geq 1}\left\|f_{j}\right\|_{B^{k}\left(\mathbb{R}^{v}\right)}<+\infty .
$$

Now, by compactness and by possibly passing to a subsequence, we may suppose $\left\{f_{j}\right\}_{j \geq 1}$ to be convergent in $L^{2}\left(\mathbb{R}^{v}\right)$. Then, again by virtue of inequality (12), we get that $\left\{f_{j}\right\}_{j \geq 1}$ is a Cauchy sequence in $B^{k}\left(\mathbb{R}^{\nu}\right)$ and finally conclude that $A_{\rho}\left(B^{k}\left(\mathbb{R}^{\nu}\right) \cap V_{1}(\rho)^{\perp}\right)$ is a closed subspace of $V_{2}(\rho)^{\perp}$. We now prove that, in fact,

$$
A_{\rho}\left(B^{k}\left(\mathbb{R}^{\nu}\right) \cap V_{1}(\rho)^{\perp}\right)=V_{2}(\rho)^{\perp} .
$$

Otherwise, if we had $A_{\rho}\left(B^{k}\left(\mathbb{R}^{v}\right) \cap V_{1}(\rho)^{\perp}\right) \neq V_{2}(\rho)^{\perp}$, we would find in the Hilbert space $V_{2}(\rho)^{\perp}$ an element $g \neq 0$ such that

$$
\left(g, A_{\rho} f\right)_{L^{2}\left(\mathbb{R}^{\nu}\right)}=0, \quad \text { for all } f \in B^{k}\left(\mathbb{R}^{\nu}\right) \cap V_{1}(\rho)^{\perp} .
$$

On the other hand, by Lemma 2.5 , we already know that

$$
\left(g, A_{\rho} f^{\prime}\right)_{L^{2}\left(\mathbb{R}^{\nu}\right)}=0, \quad \text { for all } f^{\prime} \in V_{1}(\rho) .
$$

Hence, we must have

$$
\left(g, A_{\rho} f\right)_{L^{2}\left(\mathbb{R}^{\nu}\right)}=0, \quad \text { for all } f \in B^{k}\left(\mathbb{R}^{\nu}\right) .
$$


In particular, we must have $\left(g, A_{\rho} f\right)_{L^{2}\left(\mathbb{R}^{v}\right)}=0$, for all $f \in \mathcal{S}\left(\mathbb{R}^{v}\right)$ and, hence, that $A_{\rho}^{*} g=0$ in $\mathcal{S}^{\prime}\left(\mathbb{R}^{\nu}\right)$. But then $g \in \mathcal{S}\left(\mathbb{R}^{\nu}\right)$ and $A_{\rho}^{*} g=0$, so that also $A_{\rho} A_{\rho}^{*} g=0$, i.e. $g \in \operatorname{Ker}\left(A_{\rho} A_{\rho}^{*}\right) \subset V_{2}(\rho)$. Therefore, $0 \neq g \in V_{2}(\rho)^{\perp} \cap V_{2}(\rho)$, a contradiction which proves the lemma.

As a consequence, we have that the map

$$
\left(\left.A_{\rho}\right|_{V_{1}(\rho) \perp \cap B^{k}\left(\mathbb{R}^{\nu}\right)}\right)^{-1}: V_{2}(\rho)^{\perp} \longrightarrow V_{1}(\rho)^{\perp} \cap B^{k}\left(\mathbb{R}^{\nu}\right) \quad \rho \in \tilde{U},
$$

is continuous. Upon defining $E_{\rho}: L^{2}\left(\mathbb{R}^{\nu}\right) \longrightarrow B^{k}\left(\mathbb{R}^{\nu}\right), \rho \in \tilde{U}$, by

$$
E_{\rho}= \begin{cases}0 & \text { in } V_{2}(\rho) \\ \left(\left.A_{\rho}\right|_{V_{1}(\rho) \perp \cap B^{k}\left(\mathbb{R}^{v}\right)}\right)^{-1} & \text { in } V_{2}(\rho)^{\perp},\end{cases}
$$

then $E_{\rho}$ is continuous, for all $\rho \in \tilde{U}$, and

$$
A_{\rho} E_{\rho}=1-\pi_{2}(\rho) .
$$

As a global $\psi$ do in $\mathbb{R}^{v}, \pi_{2}(\rho)$ is smoothing, for its $(x, \xi)$-symbol is, with $\left\{\phi_{j}(\rho ; .)\right\}_{1 \leq j \leq d}$ an $L^{2}\left(\mathbb{R}^{v}\right)$-orthonormal basis of $V_{2}(\rho)$,

$$
\sigma_{(x, \xi)}\left(\pi_{2}(\rho)\right)=\sum_{j=1}^{d} e^{-i x \cdot \xi} \phi_{j}(\rho ; x) \overline{\hat{\phi}_{j}(\rho ; \xi)} .
$$

It follows that $E_{\rho}$ is a globally elliptic $\psi$ do of order $-k$. We also have that

$$
E_{\rho} A_{\rho}=1-\pi_{1}(\rho)
$$

and that $E_{\rho}$ may be thought of as a continuous map from $\mathcal{S}\left(\mathbb{R}^{\nu}\right)\left(\right.$ respectively $\left.\mathcal{S}^{\prime}\left(\mathbb{R}^{\nu}\right)\right)$ into itself. Finally, exactly as in Helffer [7], one proves that $E_{\rho}$ depends in a $C^{\infty}$ fashion on $\rho \in \tilde{U}$.

A particularly meaningful case in which the bundles $V_{1}$ and $V_{2}$ can be explicitly described is the following.

Suppose

$$
\left[A_{\rho}, A_{\rho}^{*}\right]=0, \quad \text { for all } \rho \in \Sigma\left(\text { or for } \rho \text { conically near } \rho_{0}\right) .
$$

We then have the following further properties:

- $\operatorname{Ker}\left(A_{\rho}-\zeta\right)=\operatorname{Ker}\left(A_{\rho}^{*}-\bar{\zeta}\right)$, for all $\zeta \in \mathbb{C}$;

- $\zeta \neq \zeta^{\prime} \Longrightarrow \operatorname{Ker}\left(A_{\rho}-\zeta\right) \perp_{L^{2}} \operatorname{Ker}\left(A_{\rho}-\zeta^{\prime}\right)$;

- $\quad L^{2}\left(\mathbb{R}^{\nu}\right)=\bigoplus_{\zeta \in \operatorname{Spec}\left(A_{\rho}\right)} \operatorname{Ker}\left(A_{\rho}-\zeta\right)$ (orthogonal sum). 
We now take $\delta>0$ and a neighborhood $\tilde{U} \subset \mathbb{S}^{*} \Sigma$ of $\rho_{0}$ such that the following conditions hold.

(i) $\operatorname{Spec}\left(A_{\rho}\right) \subset\{\zeta \in \mathbb{C} ;|\zeta|<\delta\} \cup\{\zeta \in \mathbb{C} ;|\zeta|>\delta\}$, for all $\rho \in \tilde{U}$.

(ii) Upon setting

$$
W(\rho):=\bigoplus_{|\zeta|<\delta, \zeta \in \operatorname{Spec}\left(A_{\rho}\right)} \operatorname{Ker}\left(A_{\rho}-\zeta\right) \subset \mathcal{S}\left(\mathbb{R}^{\nu}\right) \quad \text { (orthogonal sum), }
$$

we require that $\operatorname{dim} W(\rho)=d$ for all $\rho \in \tilde{U}$, so that, in particular, $W\left(\rho_{0}\right)=$ $\operatorname{Ker}\left(A_{\rho_{0}}\right)$. We let $W=\bigsqcup_{\rho \in \tilde{U}} W(\rho) \longrightarrow \tilde{U}$ be the resulting Hermitian vectorbundle of rank $d$.

Suppose, furthermore, that the morphism $A_{\rho}: W(\rho) \longrightarrow W(\rho)$ is regular at $\rho_{0}$ (see [17]), that is to say, upon possibly shrinking $\tilde{U}$, we may find smooth sections $\phi_{j}$ of $W \rightarrow \tilde{U}, 1 \leq j \leq d$, such that

(a) $\quad\left(\phi_{j}(\rho ; .), \phi_{j^{\prime}}(\rho ; .)\right)_{L^{2}\left(\mathbb{R}^{v}\right)}=\delta_{j j^{\prime}}, 1 \leq j, j^{\prime} \leq d$, for all $\rho \in \tilde{U}$,

(b) $A_{\rho} \phi_{j}(\rho ;)=.\lambda_{j}(\rho) \phi_{j}(\rho ;),. 1 \leq j \leq d$, for all $\rho \in \tilde{U}$, for some functions $\lambda_{j} \in C^{\infty}(\tilde{U} ;\{\zeta \in \mathbb{C} ;|\zeta|<\delta\})$, with $\lambda_{j}\left(\rho_{0}\right)=0,1 \leq j \leq d$.

Since

$$
A_{\rho}^{*} A_{\rho} \phi_{j}(\rho ; .)=\left|\lambda_{j}(\rho)\right|^{2} \phi_{j}(\rho ; .), \quad 1 \leq j \leq d, \rho \in \tilde{U},
$$

we have that $A_{\rho}^{*} A_{\rho}=A_{\rho} A_{\rho}^{*}: W(\rho) \longrightarrow W(\rho)$ and that

$$
\operatorname{Spec}\left(\left.A_{\rho}^{*} A_{\rho}\right|_{W(\rho)}\right)=\left\{\left|\lambda_{j}(\rho)\right|^{2} ; 1 \leq j \leq d\right\} \subset\left[0, \delta^{2}\right), \quad \rho \in \tilde{U} .
$$

On the other hand, $A_{\rho}^{*} A_{\rho}: W(\rho)^{\perp} \cap B^{2 k}\left(\mathbb{R}^{\nu}\right) \longrightarrow W(\rho)^{\perp}$ and we claim that

$$
\operatorname{Spec}\left(\left.A_{\rho}^{*} A_{\rho}\right|_{W(\rho)^{\perp}}\right) \subset\left(\delta^{2},+\infty\right), \quad \rho \in \tilde{U} .
$$

In fact, take $0 \neq \psi \in W(\rho)^{\perp}$ and write

$$
\psi=\sum_{|\zeta|>\delta, \zeta \in \operatorname{Spec}\left(A_{\rho}\right)} \psi_{\zeta} \quad \text { (orthogonal sum). }
$$

Then $A_{\rho} \psi=\sum_{|\zeta|>\delta} \zeta \psi_{\zeta}$, so that

$$
\begin{aligned}
\left\|A_{\rho} \psi\right\|_{L^{2}\left(\mathbb{R}^{v}\right)}^{2} & =\left(\sum_{|\zeta|>\delta} \zeta \psi_{\zeta}, \sum_{\left|\zeta^{\prime}\right|>\delta} \zeta^{\prime} \psi_{\zeta^{\prime}}\right)_{L^{2}\left(\mathbb{R}^{v}\right)} \\
& =\sum_{|\zeta|>\delta}|\zeta|^{2}\left\|\psi_{\zeta}\right\|_{L^{2}\left(\mathbb{R}^{\nu}\right)}^{2}>\delta^{2} \sum_{|\zeta|>\delta}\left\|\psi_{\zeta}\right\|_{L^{2}\left(\mathbb{R}^{v}\right)}^{2}=\delta^{2}\|\psi\|_{L^{2}\left(\mathbb{R}^{v}\right)}^{2} .
\end{aligned}
$$

Hence, if $A_{\rho}^{*} A_{\rho} \psi=\mu \psi$ for some $\mu>0$ and $0 \neq \psi \in W(\rho)^{\perp}$, we obtain

$$
\mu\|\psi\|_{L^{2}\left(\mathbb{R}^{\nu}\right)}^{2}=\left(A_{\rho}^{*} A_{\rho} \psi, \psi\right)_{L^{2}\left(\mathbb{R}^{\nu}\right)}=\left\|A_{\rho} \psi\right\|_{L^{2}\left(\mathbb{R}^{v}\right)}^{2}>\delta^{2}\|\psi\|_{L^{2}\left(\mathbb{R}^{\nu}\right)}^{2},
$$

which proves the claim. 
Hence, $\tilde{U}$ is admissible and, furthermore,

$$
V_{1}(\rho)=V_{2}(\rho)=W(\rho), \quad \text { for all } \rho \in \tilde{U}
$$

It is important to observe that in this case, the matrix representing $A_{\rho}$ with respect to the basis $\left\{\phi_{j}(\rho ; .)\right\}_{1 \leq j \leq d}$ is the diagonal matrix

$$
\operatorname{diag}\left(\lambda_{1}(\rho), \ldots, \lambda_{d}(\rho)\right), \quad \rho \in \tilde{U}
$$

REMARK 2.8. A sufficient condition which ensures (14) is that the $\psi \mathrm{do}\left[A, A^{*}\right]$ has order $2 m-k-1 / 2$.

All of the previous constructions were carried out on $\tilde{U} \subset \mathbb{S}^{*} \Sigma$. Let $U \subset \Sigma$ be a conic neighborhood of $\rho_{0}$ which projects onto an admissible $\tilde{U}$ (we will say that such $U$ is itself admissible). Define for $(y, \eta) \in U$, using (10),

$$
V_{1}(y, \eta):=\mathrm{M}_{|\eta|}\left(V_{1}(y, \eta /|\eta|)\right), \quad V_{2}(y, \eta):=\mathrm{M}_{|\eta|}\left(V_{2}(y, \eta /|\eta|)\right) .
$$

We hence obtain Hermitian vector-bundles of rank $d$ (that we keep calling $V_{1}, V_{2}$ ) with fibers $V_{j}(y, \eta) \subset \mathcal{S}\left(\mathbb{R}^{v}\right), j=1,2$ :

$$
V_{j}:=\bigsqcup_{(y, \eta) \in U} V_{j}(y, \eta) \longrightarrow U, \quad j=1,2,
$$

and get the following consequences ((15)-(17)):

$$
\begin{gathered}
A_{\rho}: V_{1}(\rho) \longrightarrow V_{2}(\rho), \quad A_{\rho}^{*}: V_{2}(\rho) \longrightarrow V_{1}(\rho), \quad \text { for all } \rho \in U ; \\
A_{\rho}: V_{1}(\rho)^{\perp} \cap B^{k}\left(\mathbb{R}^{v}\right) \longrightarrow V_{2}(\rho)^{\perp}, \quad A_{\rho}^{*}: V_{2}(\rho)^{\perp} \cap B^{k}\left(\mathbb{R}^{v}\right) \longrightarrow V_{1}(\rho)^{\perp}
\end{gathered}
$$

are isomorphisms for all $\rho \in U$;

$$
E_{\rho}(\text { see (13)) is now defined for all } \rho \in U \text {. }
$$

From now on, we shall work on $U$, without further mention.

In the following, $V$ will stand, when no risk of confusion is present, for either bundle $V_{1}$ and $V_{2}$.

DEFINITION 2.9. A gauge of $V$ is a vector $\phi=\left(\phi_{1}, \ldots, \phi_{d}\right)$ of smooth orthonormal sections of $V$ having the following homogeneity property

$$
\phi_{j}\left((y, t \eta) ; t^{-1 / 2} x\right)=t^{\nu / 4} \phi_{j}((y, \eta) ; x), \quad 1 \leq j \leq d, t>0,(y, \eta) \in U, x \in \mathbb{R}^{\nu} .
$$

Note that since $V$ is a trivial bundle, the existence of a gauge is always ensured. 
Once we are given a gauge $\phi$ of $V$, we may define the following operators.

DEFINITION 2.10. Put, for every $\rho \in U$,

(i) $h_{\phi}^{-}(\rho): \mathbb{C}^{d} \longrightarrow V(\rho), h_{\phi}^{-}(\rho) \theta=\sum_{j=1}^{d} \theta_{j} \phi_{j}(\rho ;),. \theta \in \mathbb{C}^{d}$;

(ii) $h_{\phi}^{+}(\rho): \mathcal{S}^{\prime}\left(\mathbb{R}^{v}\right) \longrightarrow \mathbb{C}^{d}$,

$$
h_{\phi}^{+}(\rho) f=\left[\begin{array}{c}
\left\langle f, \overline{\phi_{1}(\rho ; .)}\right\rangle_{\mathcal{S}^{\prime}, \mathcal{S}} \\
\vdots \\
\left\langle f, \overline{\phi_{d}(\rho ; .)}\right\rangle_{\mathcal{S}^{\prime}, \mathcal{S}}
\end{array}\right] .
$$

(Here $\langle., \text {. . }\rangle_{\mathcal{S}^{\prime}, \mathcal{S}}$ denotes the $\mathcal{S}^{\prime}-\mathcal{S}$ duality.)

We collect in the next lemma (whose proof is straightforward) a few useful properties of the $h_{\phi}^{ \pm}$.

LEMMA 2.11.

(a) Let $\phi, \psi$ be gauges of $V$ (not necessarily the same $V$ ). Then $h_{\psi}^{+}(\rho) \circ$ $h_{\phi}^{-}(\rho): \mathbb{C}^{d} \longrightarrow \mathbb{C}^{d}$ is given by

$$
h_{\psi}^{+}(\rho) \circ h_{\phi}^{-}(\rho): \theta \longmapsto\left[\begin{array}{c}
\sum_{j=1}^{d}\left(\phi_{j}(\rho ; .), \psi_{1}(\rho ; .)\right)_{L^{2}\left(\mathbb{R}^{v}\right)} \theta_{j} \\
\vdots \\
\sum_{j=1}^{d}\left(\phi_{j}(\rho ; .), \psi_{d}(\rho ; .)\right)_{L^{2}\left(\mathbb{R}^{v}\right)} \theta_{j}
\end{array}\right] .
$$

(b) Upon denoting by $\pi(\rho): L^{2}\left(\mathbb{R}^{v}\right) \longrightarrow V(\rho)$ the orthogonal projection onto $V(\rho)$, we have

$$
h_{\phi}^{-}(\rho) \circ h_{\phi}^{+}(\rho)=\pi(\rho), \quad \text { for all } \rho \in U .
$$

(c) Let $\phi, \psi$ be gauges of the same $V$. Then $h_{\phi}^{-}(\rho)=h_{\psi}^{-}(\rho) \circ \gamma(\rho), h_{\phi}^{+}(\rho)=$ $\gamma(\rho)^{*} \circ h_{\psi}^{+}(\rho)$, where $\gamma(\rho)$ is the $d \times d$ smooth (in $\rho$ ) unitary matrix defined by the relations

$$
\phi_{j}(\rho ; .)=\sum_{j^{\prime}=1}^{d} \gamma_{j^{\prime} j}(\rho) \psi_{j^{\prime}}(\rho ; .), \quad j=1, \ldots, d .
$$

Following Sjöstrand and Helffer, we next define on $U$ an object which takes care of both the localized operator $A_{\rho}$ and the subspaces $V_{1}(\rho), V_{2}(\rho)$, for $\rho \in U$. 
DEFINITION 2.12. For a given gauge $\phi$ of $V_{2}$ and $\phi^{\prime}$ of $V_{1}$, define, for every $\rho \in U$,

$$
\begin{aligned}
\mathcal{A}_{\rho, \phi, \phi^{\prime}}= & {\left[\begin{array}{cc}
A_{\rho} & h_{\phi}^{-}(\rho) \\
h_{\phi^{\prime}}^{+}(\rho) & 0
\end{array}\right] \begin{array}{cc}
\mathcal{S}\left(\mathbb{R}^{v}\right) & \mathcal{S}\left(\mathbb{R}^{v}\right) \\
: & \times \\
\mathbb{C}^{d} & \times \\
\mathbb{C}^{d}
\end{array}, } \\
& \mathcal{A}_{\rho, \phi, \phi^{\prime}}\left[\begin{array}{l}
f \\
\theta
\end{array}\right]:=\left[\begin{array}{c}
A_{\rho} f+h_{\phi}^{-}(\rho) \theta \\
h_{\phi^{\prime}}^{+}(\rho) f
\end{array}\right] .
\end{aligned}
$$

We have the following crucial result.

THEOREM 2.13. $\mathcal{A}_{\rho, \phi, \phi^{\prime}}$ is invertible for all $\rho \in U$, with its inverse given by

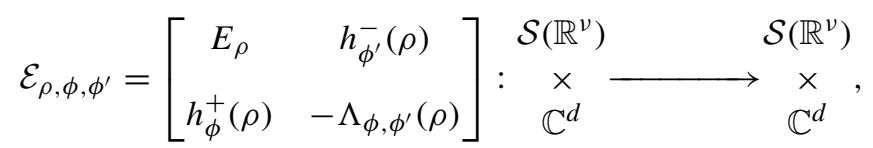

where $E_{\rho}: L^{2}\left(\mathbb{R}^{v}\right) \longrightarrow B^{k}\left(\mathbb{R}^{v}\right), \rho \in U$, is the operator defined in (13) and (17), i.e. upon writing $f=f_{\rho}^{\prime}+f_{\rho}^{\prime \prime}$ with $f_{\rho}^{\prime} \in V_{2}(\rho)$ and $f_{\rho}^{\prime \prime} \in V_{2}(\rho)^{\perp}$,

$$
E_{\rho} f=\left(\left.A_{\rho}\right|_{V_{1}(\rho)^{\perp} \cap B^{k}\left(\mathbb{R}^{v}\right)}\right)^{-1} f_{\rho}^{\prime \prime} ;
$$

and $\Lambda_{\phi, \phi^{\prime}}(\rho)$ is the $d \times d$ smooth matrix defined by

$$
\Lambda_{\phi, \phi^{\prime}}(\rho)=\left(h_{\phi}^{+}(\rho) \circ A_{\rho} \circ h_{\phi^{\prime}}^{-}(\rho)\right), \quad \rho \in U .
$$

Proof. To prove injectivity, we suppose $A_{\rho} f+h_{\phi}^{-}(\rho) \theta=0$ and $h_{\phi^{\prime}}^{+}(\rho) f=0$. Then $f \in V_{1}(\rho)^{\perp}$ so that $A_{\rho} f \in V_{2}(\rho)^{\perp}$. Since $h_{\phi}^{-}(\rho) \theta \in V_{2}(\rho)$, we conclude that $A_{\rho} f=0=h_{\phi}^{-}(\rho) \theta$, whence $f=0, \theta=0$.

To prove surjectivity, suppose we are given $g \in \mathcal{S}\left(\mathbb{R}^{v}\right)$ and $\omega \in \mathbb{C}^{d}$. We have to solve the system

$$
\left\{\begin{array}{l}
A_{\rho} f+h_{\phi}^{-}(\rho) \theta=g \\
h_{\phi^{\prime}}^{+}(\rho) f=\omega .
\end{array}\right.
$$

We look for $f=h_{\phi^{\prime}}^{-}(\rho) \omega+f^{\prime}$, with $f^{\prime} \in V_{1}(\rho)^{\perp}$ to be determined. By definition, it is then true that $h_{\phi^{\prime}}^{+}(\rho) f=\omega$. The first equation in (24) is now

$$
A_{\rho} f+h_{\phi}^{-}(\rho) \theta=\left(A_{\rho} \circ h_{\phi^{\prime}}^{-}(\rho)\right) \omega+A_{\rho} f^{\prime}+h_{\phi}^{-}(\rho) \theta=g .
$$

To solve this equation, we impose the condition

$$
g-\left(A_{\rho} \circ h_{\phi^{\prime}}^{-}(\rho)\right) \omega-h_{\phi}^{-}(\rho) \theta \in V_{2}(\rho)^{\perp},
$$


i.e.

$$
h_{\phi}^{+}(\rho)\left(g-\left(A_{\rho} \circ h_{\phi^{\prime}}^{-}(\rho)\right) \omega-h_{\phi}^{-}(\rho) \theta\right)=0,
$$

which gives

$$
\left(h_{\phi}^{+}(\rho) \circ h_{\phi}^{-}(\rho)\right) \theta=\theta=h_{\phi}^{+}(\rho) g-\left(h_{\phi}^{+}(\rho) \circ A_{\rho} \circ h_{\phi^{\prime}}^{-}(\rho)\right) \omega
$$

and, hence, we can find the unique $\theta \in \mathbb{C}^{d}$ satisfying the previous equation. Note that $g-\left(A_{\rho} \circ h_{\phi^{\prime}}^{-}(\rho)\right) \omega-h_{\phi}^{-}(\rho) \theta \in \mathcal{S}\left(\mathbb{R}^{\nu}\right)$. As a consequence, we have

$$
f^{\prime}=\left(\left.A_{\rho}\right|_{V_{1}(\rho)^{\perp} \cap B^{k}\left(\mathbb{R}^{v}\right)}\right)^{-1}\left(g-\left(A_{\rho} \circ h_{\phi^{\prime}}^{-}(\rho)\right) \omega-h_{\phi}^{-}(\rho) \theta\right) .
$$

Note also that $f^{\prime} \in \mathcal{S}\left(\mathbb{R}^{\nu}\right)$ by virtue of the ellipticity of $A_{\rho}$.

Since

$$
E_{\rho}= \begin{cases}0 & \text { on } V_{2}(\rho) \\ \left(\left.A_{\rho}\right|_{V_{1}(\rho) \perp \cap B^{k}\left(\mathbb{R}^{v}\right)}\right)^{-1} & \text { on } V_{2}(\rho)^{\perp},\end{cases}
$$

and being $\left(A_{\rho} \circ h_{\phi^{\prime}}^{-}(\rho)\right) \omega+h_{\phi}^{-}(\rho) \theta \in V_{2}(\rho)$, we finally have

$$
\left\{\begin{array}{l}
f=E_{\rho} g+h_{\phi^{\prime}}^{-}(\rho) \omega \\
\theta=h_{\phi}^{+}(\rho) g-\Lambda_{\phi, \phi^{\prime}}(\rho) \omega .
\end{array}\right.
$$

This concludes the proof.

REMARK 2.14.

1) In exactly the same way as the theorem was proven, one can show that $\mathcal{E}_{\rho, \phi, \phi^{\prime}}$ is the inverse of $\mathcal{A}_{\rho, \phi, \phi^{\prime}}$ when the latter is considered as acting on $\mathcal{S}^{\prime}\left(\mathbb{R}^{\nu}\right) \times \mathbb{C}^{d}$ into itself.

2) Note that if $\tilde{\phi}$, respectively $\tilde{\phi}^{\prime}$, is another gauge of $V_{2}$, respectively $V_{1}$, then

$$
\mathcal{E}_{\rho, \tilde{\phi}, \tilde{\phi}^{\prime}}=\left[\begin{array}{cc}
\text { id } & 0 \\
0 & \gamma(\rho)^{-1}
\end{array}\right] \mathcal{E}_{\rho, \phi, \phi^{\prime}}\left[\begin{array}{cc}
\text { id } & 0 \\
0 & \left(\gamma^{\prime}(\rho)^{-1}\right)^{*}
\end{array}\right],
$$

where $\gamma$, respectively $\gamma^{\prime}$, is the unitary matrix for which $h_{\tilde{\phi}}^{-}(\rho)=h_{\phi}^{-}(\rho) \circ \gamma(\rho)$, respectively $h_{\tilde{\phi}^{\prime}}^{-}(\rho)=h_{\phi^{\prime}}^{-}(\rho) \circ \gamma^{\prime}(\rho)$.

3) The $d \times d$ matrix $\Lambda_{\phi, \phi^{\prime}}(\rho)=h_{\phi}^{+}(\rho) \circ A_{\rho} \circ h_{\phi^{\prime}}^{-}(\rho)$ is smooth in $\rho$ and it has entries

$$
\Lambda_{\phi, \phi^{\prime}}^{\left(j j^{\prime}\right)}(\rho)=\left(A_{\rho} \phi_{j^{\prime}}^{\prime}(\rho ; .), \phi_{j}(\rho ; .)\right)_{L^{2}\left(\mathbb{R}^{\nu}\right)}, \quad 1 \leq j, j^{\prime} \leq d .
$$


Note that $\Lambda_{\phi, \phi^{\prime}}\left(\rho_{0}\right)=0$. In general, the previous matrix may also be singular for $\rho \neq \rho_{0}, \rho \in U$. As a matter of fact,

$$
\operatorname{Ker}\left(\Lambda_{\phi, \phi^{\prime}}(\rho)\right)=\left\{\theta \in \mathbb{C}^{d} ; h_{\phi^{\prime}}^{-}(\rho) \theta \in \operatorname{Ker}\left(A_{\rho}\right)\right\} .
$$

Hence, the matrix is invertible at $\rho \neq \rho_{0}$ exactly when $\operatorname{Ker}\left(A_{\rho}\right)=\{0\}$.

4) Since

$$
\left[\begin{array}{cc}
A_{\rho} & h_{\phi}^{-}(\rho) \\
h_{\phi^{\prime}}^{+}(\rho) & 0
\end{array}\right]^{*}=\left[\begin{array}{cc}
A_{\rho}^{*} & h_{\phi^{\prime}}^{-}(\rho) \\
h_{\phi}^{+}(\rho) & 0
\end{array}\right] \text {, }
$$

then

$$
\left[\begin{array}{cc}
E_{\rho}^{*} & h_{\phi}^{-}(\rho) \\
h_{\phi^{\prime}}^{+}(\rho) & -\Lambda_{\phi, \phi^{\prime}}(\rho)^{*}
\end{array}\right]
$$

is the inverse of

$$
\left[\begin{array}{cc}
A_{\rho}^{*} & h_{\phi^{\prime}}^{-}(\rho) \\
h_{\phi}^{+}(\rho) & 0
\end{array}\right] \text {. }
$$

We end this section by giving an idea of the strategy that will be followed below. Exactly in the same way as the invertibility of the localized operators $A_{\rho}$ allows a construction of an actual parametrix of $A$, the invertibility of the matrices $\mathcal{A}_{\rho, \phi, \phi^{\prime}}$ will allow the construction of a parametrix of a suitable system of $\psi$ dos in all the variables, of which $\mathcal{A}_{\rho, \phi, \phi^{\prime}}$ is the associated localized operator. This construction requires a proper pseudodifferential calculus. Fortunately, the core of the calculus has already been developed by Boutet de Monvel in [3] (see also Helffer [7]) and, in the next section, we just recall Boutet's calculus emphasizing the homogeneity properties and the related asymptotic expansions.

REMARK 2.15. At this point, at last, we are in a position to comment on Helffer's setting in [7], that of Grigis and Rothschild in [5] and to motivate our own setting.

In [7], Helffer considers an a priori more general case, namely a system of the form (19) where he only requires that $\phi$, respectively $\phi^{\prime}$, at $\rho_{0}$ be bases of $\operatorname{Ker}\left(A_{\rho_{0}}^{*}\right)$, respectively $\operatorname{Ker}\left(A_{\rho_{0}}\right)$ (spaces that he does not require to have the same dimension). From the invertibility of $\mathcal{A}_{\rho_{0}, \phi, \phi^{\prime}}$, a perturbation argument yields the invertibility of $\mathcal{A}_{\rho, \phi, \phi^{\prime}}$ in a neighborhood of $\rho_{0}$. The inverse has the form $\mathcal{E}_{\rho, \psi, \psi^{\prime}}$ for suitable $\psi, \psi^{\prime}$, which, however, in general coincide with $\phi, \phi^{\prime}$, respectively, only at $\rho_{0}$. At any rate, this more general setup ensures control only on the principal symbol $\Lambda_{m-k / 2}(y, \eta)$ of the systems $\Lambda\left(y, D_{y}\right)$, allowing him to obtain hypoellipticity results with loss of 
$k / 2+r /(r+1)$ derivatives, $r$ being a positive integer. In particular, it seems to us that, in his setting, cases in which $\Lambda_{m-k / 2}(y, \eta)$ vanishes identically or vanishes to order $\geq 2$ on some submanifold of $\Sigma$ are out of reach. Our motivation is precisely to be able to treat these latter cases, which, in principle, require knowledge of the complete symbol of $\Lambda\left(y, D_{y}\right)$. To get the full expansion of the symbol at each 'order of homogeneity', we have to solve systems of the form $\mathcal{A}_{\rho, \phi, \phi^{\prime}}$ exactly, and for that we have decided to introduce hypothesis (H1) and work with the bundles $V_{1}$ and $V_{2}$. A consequence (and maybe a drawback) of our approach is that we will obtain the hypoellipticity of $A$ and $A^{*}$ at the same time.

As for Grigis and Rothschild, our approach is similar (at least in spirit) to theirs; however, they still use a perturbation argument which is strictly bound to treating 'translation-invariant' operators (i.e. operators whose symbol does not depend on y).

\section{The calculus}

3.1. $S^{m, k}$ classes

We start off by recalling what Boutet's $S^{m, k}$ classes (see [3]) are in our situation.

DEFINITION 3.1. Let $m, k \in \mathbb{R}$. By $S^{m, k}$ we denote the class of all smooth functions $a(z=(x, y), \zeta=(\xi, \eta))=a: T^{*} \mathbb{R}^{n} \longrightarrow \mathbb{C}$ such that the following inequalities hold for all multi-indices $\alpha, \beta, \gamma, \delta$ :

$$
\left|\partial_{x}^{\alpha} \partial_{y}^{\beta} \partial_{\xi}^{\gamma} \partial_{\eta}^{\delta} a(z, \zeta)\right| \lesssim|\zeta|^{m-|\gamma|-|\delta|}\left(|x|+\frac{|\xi|}{|\zeta|}+\frac{1}{|\zeta|^{1 / 2}}\right)^{k-|\alpha|-|\gamma|} .
$$

By $a\left(z, D_{z}\right)$, we denote the corresponding (properly supported) $\psi$ do and, by $\mathrm{OPS}^{m, k}$, the corresponding class of $\psi \mathrm{dos}$. We put

$$
S^{m, \infty}:=\bigcap_{k \in \mathbb{R}} S^{m, k}
$$

REMARK 3.2. One has the following useful properties (see [3]).

(i) $S^{m, k} \subset S_{1 / 2,1 / 2}^{m+k_{-} / 2}, k_{-}=\max \{0,-k\}$;

(ii) $S^{m, k} \subset S^{m^{\prime}, k^{\prime}} \Longleftrightarrow m \leq m^{\prime}$ and $m-\frac{1}{2} k \leq m^{\prime}-\frac{1}{2} k^{\prime}$.

We will use the 'homogeneous' analogue of the classes $S^{m, k}$.

DEFINITION 3.3.

(a) Let $k \in \mathbb{R}$. By $\mathbf{S}^{k}$ we denote the class of all smooth functions $b(x, \xi)=$ $b: T^{*} \mathbb{R}^{v} \longrightarrow \mathbb{C}$ satisfying the following global inequalities for all multi-indices $\alpha, \beta$

$$
\left|\partial_{x}^{\alpha} \partial_{\xi}^{\beta} b(x, \xi)\right| \leq C_{\alpha \beta}(1+|x|+|\xi|)^{k-|\alpha|-|\beta|}
$$

(see Schubin [18] or Helffer [8]). 
By $\mathrm{OPS}^{k}$, we denote the class of the corresponding global $\psi \mathrm{dos}$ (as acting from $\mathcal{S}\left(\mathbb{R}^{v}\right)$, respectively $\mathcal{S}^{\prime}\left(\mathbb{R}^{v}\right)$, into itself). Given $a\left(x, D_{x}\right) \in \operatorname{OPS}^{k}$ and $b\left(x, D_{x}\right) \in$ OPS $^{k^{\prime}}$, it is well known that their composition, hereafter denoted by $a\left(x, D_{x}\right) \bullet$ $b\left(x, D_{x}\right)$, belongs to OPS ${ }^{k+k^{\prime}}$. Moreover, the symbol of the composition, hereafter denoted by $\sigma_{(x, \xi)}(a \bullet b)$, is given (as an oscillatory integral) by

$$
\sigma_{(x, \xi)}(a \bullet b)=\iint e^{-i s \cdot \tau} a(x, \xi+\tau) b(x+s, \xi) d s d \tau \quad(x, \xi) \in T^{*} \mathbb{R}^{v}
$$

Here $d \tau=(2 \pi)^{-v} d \tau$.

(b) Let $m, k \in \mathbb{R}$. By $S_{\mathrm{hom}}^{m, k}$, we denote the class of all smooth functions $a(y, \eta ; x, \xi)=a:\left(T^{*} \mathbb{R}^{n-v} \backslash 0\right) \times T^{*} \mathbb{R}^{v} \longrightarrow \mathbb{C}$ such that

(i) $\quad a(y, \eta ;.) \in \mathbf{S}^{k}$, for all $(y, \eta) \in T^{*} \mathbb{R}^{n-v} \backslash 0$;

(ii) $a\left(y, t \eta ; t^{-1 / 2} x, t^{1 / 2} \xi\right)=t^{m-k / 2} a(y, \eta ; x, \xi), t>0$.

It is important to notice that any given symbol $a \in S_{\mathrm{hom}}^{m, k}$, when multiplied by any excision function $\chi=\chi(\eta)$ (i.e. $\chi$ is smooth, vanishes in a bounded neighborhood of zero and is identically one outside a bounded neighborhood of zero), gives a symbol $\chi a \in S^{m, k}$. This fact will hereafter be denoted simply by $S_{\mathrm{hom}}^{m, k} \subset S^{m, k}$, so that when thinking of $a \in S_{\mathrm{hom}}^{m, k}$ as an element of $S^{m, k}$, we shall tacitly assume it was previously multiplied by our favorite excision function.

(c) By $\mathrm{OPS}_{\mathrm{cl}}^{m, k}$ we denote the class of operators $a\left(z, D_{z}\right) \in \mathrm{OPS}^{m, k}$ such that there exists a formal series $\sum_{j \geq 0} a_{m-j / 2}(y, \eta ; x, \xi)$ of symbols $a_{m-j / 2} \in S_{\mathrm{hom}}^{m, k+j}$ such that

$$
a \sim \sum_{j \geq 0} a_{m-j / 2}
$$

i.e.

$$
a-\sum_{j=0}^{N-1} a_{m-j / 2} \in S^{m, k+N}, \quad \text { for all } N \geq 1 .
$$

The following lemma is crucial (see [3]).

LEMMA 3.4. Given a formal series $\sum_{j \geq 0} a_{m-j / 2}, a_{m-j / 2} \in S_{\mathrm{hom}}^{m, k+j}$, there exists $a\left(z, D_{z}\right) \in \mathrm{OPS}_{\mathrm{cl}}^{m, k}$, uniquely determined modulo $\mathrm{OPS}^{m, \infty}$, such that $a \sim$ $\sum_{j \geq 0} a_{m-j / 2}$.

The main motivation for introducing $\mathrm{OPS}_{\mathrm{cl}}^{m, k}$ is given by the following remark.

REMARK 3.5. Suppose we are given a $\psi$ do $A \in \operatorname{OPS}_{\mathrm{cl}}^{m}\left(\mathbb{R}^{n}\right)$ with symbol $\sigma(A)$ having semi-regular asymptotic expansion $\sigma(A) \sim \sum_{r \geq 0} a_{m-r / 2}(z, \zeta)$, and such that, for 
some $k \in \mathbb{Z}_{+}, a_{m-r / 2}$ vanishes at $\Sigma$ to order $k-r, 0 \leq r \leq k$. Then it is readily checked that $A \in \mathrm{OPS}_{\mathrm{cl}}^{m, k}$ with

$$
\sigma(A) \sim \sum_{j \geq 0} \tilde{a}_{m-j / 2}(y, \eta ; x, \xi)
$$

where

$$
\tilde{a}_{m-j / 2}(y, \eta ; x, \xi)=\sum_{|\alpha|+|\beta|+r=k+j} \frac{1}{\alpha ! \beta !}\left(\partial_{x}^{\alpha} \partial_{\xi}^{\beta} a_{m-r / 2}\right)(x=0, y, \xi=0, \eta) x^{\alpha} \xi^{\beta}
$$

It is worth noting that (at least when $k \geq 1) \tilde{a}_{m}(y, \eta ; x, \xi)$ is $a_{(y, \eta)}(x, \xi)$, as defined in (4).

The following composition result will be systematically used throughout.

LEMMA 3.6. Let $a\left(z, D_{z}\right) \in \mathrm{OPS}_{\mathrm{cl}}^{m, k}, b\left(z, D_{z}\right) \in \mathrm{OPS}_{\mathrm{cl}}^{m^{\prime}, k^{\prime}}$, where $a \sim \sum_{j \geq 0}$ $a_{m-j / 2}, b \sim \sum_{j \geq 0} b_{m^{\prime}-j / 2}$. Then, modulo $\mathrm{OPS}^{m+m^{\prime}, \infty}$,

$$
a\left(z, D_{z}\right) b\left(z, D_{z}\right)=c\left(z, D_{z}\right) \in \operatorname{OPS}_{\mathrm{cl}}^{m+m^{\prime}, k+k^{\prime}},
$$

with $c \sim \sum_{r \geq 0} c_{m+m^{\prime}-r / 2}$, and the $c_{m+m^{\prime}-r / 2}$ given by

$$
\begin{aligned}
& c_{m+m^{\prime}-r / 2}(y, \eta ; x, \xi) \\
& \quad=\sum_{2|\alpha|+j+j^{\prime}=r} \frac{1}{\alpha ! i^{|\alpha|}} \sigma_{(x, \xi)}\left(\left(\partial_{\eta}^{\alpha} a_{m-j / 2}\right)\left(y, \eta ; x, D_{x}\right) \bullet\left(\partial_{y}^{\alpha} b_{m^{\prime}-j^{\prime} / 2}\right)\left(y, \eta ; x, D_{x}\right)\right),
\end{aligned}
$$

where, recalling Definition $3.3(a), \sigma_{(x, \xi)}\left(\partial_{\eta}^{\alpha} a_{m-j / 2} \bullet \partial_{y}^{\alpha} b_{m^{\prime}-j^{\prime} / 2}\right)$ denotes the symbol in $\mathbf{S}^{k+k^{\prime}+j+j^{\prime}}$ of the composition.

We omit the proof of the lemma.

\subsection{Hermite operators}

We start out this subsection by recalling, from Boutet's paper [3], the definition of another important class of operators.

Definition 3.7. Let $\mu \in \mathbb{R}$. By $\mathbf{H}^{\mu}$ we denote the class of all smooth functions $\varphi(y, \eta ; x)=\varphi: T^{*} \mathbb{R}^{n-v} \times \mathbb{R}^{v} \longrightarrow \mathbb{C}$ such that the following inequalities hold for all multi-indices $\alpha, \beta, \gamma$ and all integers $N \in \mathbb{Z}_{+}$:

$$
\left|\partial_{x}^{\alpha} \partial_{y}^{\beta} \partial_{\eta}^{\gamma} \varphi(y, \eta ; x)\right| \lesssim|\eta|^{\mu+\nu / 4-|\gamma|+|\alpha| / 2-N / 2}\left(|x|+\frac{1}{|\eta|^{1 / 2}}\right)^{-N} .
$$


By

$$
\varphi\left(y, D_{y} ; x\right): C_{0}^{\infty}\left(\mathbb{R}^{n-v}\right) \longrightarrow C^{\infty}\left(\mathbb{R}^{n}\right)
$$

we denote the $\psi \mathrm{do}$

$$
\varphi\left(y, D_{y} ; x\right) f=\int e^{i y \cdot \eta} \varphi(y, \eta ; x) \hat{f}(\eta) d \eta
$$

and by $\mathrm{OPH}^{\mu}$ the class of $\psi$ dos of the form $\varphi\left(y, D_{y} ; x\right)+R$, where $\varphi \in \mathbf{H}^{\mu}$ and $R: \mathcal{E}^{\prime}\left(\mathbb{R}^{n-v}\right) \longrightarrow C^{\infty}\left(\mathbb{R}^{n}\right)$ is a smoothing operator.

As in the former subsection, we have a corresponding 'homogeneous' version.

DEFINITION 3.8. Let $\mu \in \mathbb{R}$. By $\mathbf{H}_{\text {hom }}^{\mu}$ we denote the class of all smooth functions $\varphi(y, \eta ; x)=\varphi: T^{*} \mathbb{R}^{n-v} \backslash 0 \times \mathbb{R}^{v} \longrightarrow \mathbb{C}$ such that

(i) $\quad \varphi(y, \eta ;.) \in \mathcal{S}\left(\mathbb{R}^{\nu}\right)$, for all $(y, \eta) \in T^{*} \mathbb{R}^{n-\nu} \backslash 0$;

(ii) $\varphi\left(y, t \eta ; t^{-1 / 2} x\right)=t^{\mu+v / 4} \varphi(y, \eta ; x), t>0$.

Observe that $\mathbf{H}_{\text {hom }}^{\mu} \subset \mathbf{H}^{\mu}$, in the sense that $\varphi \in \mathbf{H}_{\text {hom }}^{\mu}$ yields $\chi(\eta) \varphi(y, \eta ; x) \in \mathbf{H}^{\mu}$ for every excision function $\chi$. By $\mathrm{OPH}_{\mathrm{cl}}^{\mu}$, we denote those operators $\varphi\left(y, D_{y} ; x\right) \in \mathrm{OPH}^{\mu}$ for which there exists a formal series $\sum_{j \geq 0} \varphi_{\mu-j / 2}$, with $\varphi_{\mu-j / 2} \in \mathbf{H}_{\mathrm{hom}}^{\mu-j / 2}$, such that $\varphi \sim \sum_{j \geq 0} \varphi_{\mu-j / 2}$, i.e.

$$
\varphi-\sum_{j=0}^{N-1} \varphi_{\mu-j / 2} \in \mathbf{H}^{\mu-N / 2}, \quad \text { for all } N \geq 1 .
$$

We call $\varphi_{\mu}$ the principal symbol of $\varphi\left(y, D_{y} ; x\right)$.

There is an analogue of Lemma 3.4, which we state without proof.

Lemma 3.9. Given any formal series $\sum_{j \geq 0} \varphi_{\mu-j / 2}, \varphi_{\mu-j / 2} \in \mathbf{H}_{\mathrm{hom}}^{\mu-j / 2}$, there exists $\varphi\left(y, D_{y} ; x\right) \in \mathrm{OPH}_{\mathrm{cl}}^{\mu}$ with $\varphi \sim \sum_{j \geq 0} \varphi_{\mu-j / 2}$.

As the attentive reader may have already noticed, the motivation for introducing the classes $\mathbf{H}_{\text {hom }}^{\mu}$ is that the eigenfunctions of the localized operator $A_{(y, \eta)}$ of the Introduction are, in fact, smooth in $(y, \eta), \eta \neq 0$, and rapidly decreasing in $x$ (see also Definition 2.9). Moreover, the Hermite operators $\varphi\left(y, D_{y} ; x\right)$ are the natural quantized version in all the variables of the operators $h_{\phi}^{-}$of Section 2.

If we want to quantize in all the variables an eigenvalue equation

$$
A_{(y, \eta)}\left(x, D_{x}\right)(\phi(y, \eta ; .))=\lambda(y, \eta) \phi(y, \eta ; x),
$$

we need to understand the composition between $\mathrm{OPS}_{\mathrm{cl}}^{m, k}$ and $\mathrm{OPH}_{\mathrm{cl}}^{\mu}$ and the composition between $\mathrm{OPH}_{\mathrm{cl}}^{\mu}$ and $\mathrm{OPS}_{\mathrm{cl}}^{m-k / 2}\left(\mathbb{R}^{n-\nu}\right)$. This is taken care of by the following lemma (whose proof is left to the reader). 
LEMMA 3.10.

1) Let $a\left(z, D_{z}\right) \in \mathrm{OPS}_{\mathrm{cl}}^{m, k}$ with $a \sim \sum_{j \geq 0} a_{m-j / 2}$, and let $\varphi\left(y, D_{y} ; x\right) \in \mathrm{OPH}_{\mathrm{cl}}^{\mu}$, with $\varphi \sim \sum_{j \geq 0} \varphi_{\mu-j / 2}$. Then

$$
a\left(z, D_{z}\right) \varphi\left(y, D_{y} ; x\right)=\psi\left(y, D_{y} ; x\right) \in \mathrm{OPH}_{\mathrm{cl}}^{\mu+m-k / 2},
$$

with $\psi \sim \sum_{\ell \geq 0} \psi_{\mu+m-k / 2-\ell / 2}$, where

$$
\begin{aligned}
& \psi_{\mu+m-k / 2-\ell / 2}(y, \eta ; x) \\
& \quad=\sum_{2|\alpha|+j+r=\ell} \frac{1}{\alpha ! i|\alpha|}\left(\partial_{\eta}^{\alpha} a_{m-j / 2}\right)\left(y, \eta ; x, D_{x}\right)\left(\partial_{y}^{\alpha} \varphi_{\mu-r / 2}(y, \eta ; .)\right) .
\end{aligned}
$$

2) Let $\varphi\left(y, D_{y} ; x\right)$ be as in 1$)$. Let $b\left(y, D_{y}\right) \in \mathrm{OPS}_{\mathrm{cl}}^{m^{\prime}}\left(\mathbb{R}^{n-v}\right)$ with symbol $b(y, \eta) \sim \sum_{j \geq 0} b_{m^{\prime}-j / 2}(y, \eta)$. Then

$$
\varphi\left(y, D_{y} ; x\right) b\left(y, D_{y}\right)=\psi\left(y, D_{y} ; x\right) \in \mathrm{OPH}_{\mathrm{cl}}^{\mu+m^{\prime}},
$$

with $\psi \sim \sum_{\ell \geq 0} \psi_{\mu+m^{\prime}-\ell / 2}$, where

$$
\psi_{\mu+m^{\prime}-\ell / 2}(y, \eta ; x)=\sum_{2|\alpha|+j+r=\ell} \frac{1}{\alpha ! i^{|\alpha|}}\left(\partial_{\eta}^{\alpha} \varphi_{\mu-j / 2}\right)(y, \eta ; x)\left(\partial_{y}^{\alpha} b_{m^{\prime}-r / 2}\right)(y, \eta) .
$$

As mentioned earlier, the class $\mathrm{OPH}_{\mathrm{cl}}^{\mu}$ represents the natural quantization of the operators $h_{\phi}^{-}$. As regards quantization of the operators $h_{\phi}^{+}$, we proceed as follows.

Definition 3.11. Let $\mu \in \mathbb{R}$ and let $\varphi \in \mathbf{H}^{\mu}$ have the asymptotic expansion $\varphi \sim \sum_{\ell \geq 0} \varphi_{\mu-\ell / 2}, \varphi_{\mu-\ell / 2} \in \mathbf{H}_{\mathrm{hom}}^{\mu-\ell / 2} . B y$

$$
\varphi^{*}\left(y, D_{y} ; x\right): C_{0}^{\infty}\left(\mathbb{R}^{n}\right) \longrightarrow C^{\infty}\left(\mathbb{R}^{n-v}\right)
$$

we denote the operator

$$
C_{0}^{\infty}\left(\mathbb{R}_{x}^{v} \times \mathbb{R}_{y}^{n-v}\right) \ni g \longmapsto \varphi^{*}\left(y, D_{y} ; x\right) g=\iint e^{i y \cdot \eta} \overline{\varphi(y, \eta ; x)} \hat{g}(x, \eta) d \eta d x
$$

$(\hat{g}(x, \eta)$ denotes partial Fourier transform with respect to $y)$.

By $\mathrm{OPH}_{\mathrm{cl}}^{* \mu}$ we denote the class of all operators of the form $\varphi^{*}\left(y, D_{y} ; x\right)+R$, where $R: \mathcal{E}^{\prime}\left(\mathbb{R}^{n}\right) \longrightarrow C^{\infty}\left(\mathbb{R}^{n-v}\right)$ is a smoothing operator and we call $\varphi_{\mu}$ the principal symbol of $\varphi^{*}\left(y, D_{y} ; x\right)$.

In the following lemma, we gather some composition results that will be crucial later. (The proof is, of course, left to the reader.) 
LEMMA 3.12.

1) Let $a\left(z, D_{z}\right) \in \mathrm{OPS}_{\mathrm{cl}}^{m, k}$ with $a \sim \sum_{j \geq 0} a_{m-j / 2}$, and let $\varphi^{*}\left(y, D_{y} ; x\right) \in$ $\mathrm{OPH}_{\mathrm{cl}}^{* \mu}$ with $\varphi \sim \sum_{\ell \geq 0} \varphi_{\mu-\ell / 2}$. Then

$$
\varphi^{*}\left(y, D_{y} ; x\right) a\left(z, D_{z}\right)=\psi^{*}\left(y, D_{y} ; x\right) \in \mathrm{OPH}_{\mathrm{cl}}^{* \mu+m-k / 2},
$$

with $\psi \sim \sum_{r \geq 0} \psi_{\mu+m-k / 2-r / 2}$, where

$$
\begin{aligned}
& \psi_{\mu+m-k / 2-r / 2}(y, \eta ; x) \\
& \quad=\sum_{2|\alpha|+j+\ell=r} \frac{i^{|\alpha|}}{\alpha !} \overline{\left(\partial_{y}^{\alpha} a_{m-j / 2}\right)\left(y, \eta ; x, D_{x}\right)^{*}\left(\left(\partial_{\eta}^{\alpha} \varphi_{\mu-\ell / 2}\right)(y, \eta ; .)\right)},
\end{aligned}
$$

where $\left(\partial_{y}^{\alpha} a_{m-j / 2}\right)\left(y, \eta ; x, D_{x}\right)^{*} \in \mathrm{OPS}^{k+j}$ is the formal adjoint of the operator $\left(\partial_{y}^{\alpha} a_{m-j / 2}\right)\left(y, \eta ; x, D_{x}\right)$.

2) Let $\varphi^{*}\left(y, D_{y} ; x\right)$ be as before and let $b\left(y, D_{y}\right) \in \operatorname{OPS}_{\mathrm{cl}}^{m^{\prime}}\left(\mathbb{R}^{n-\nu}\right)$ with $b \sim$ $\sum_{j \geq 0} b_{m^{\prime}-j / 2}$. Then

$$
b\left(y, D_{y}\right) \varphi^{*}\left(y, D_{y} ; x\right)=\psi^{*}\left(y, D_{y} ; x\right) \in \mathrm{OPH}_{\mathrm{cl}}^{* \mu+m^{\prime}},
$$

with $\psi \sim \sum_{r \geq 0} \psi_{\mu+m^{\prime}-r / 2}$, where

$$
\psi_{\mu+m^{\prime}-r / 2}(y, \eta ; x)=\sum_{2|\alpha|+j+\ell=r} \frac{i^{|\alpha|}}{\alpha !} \overline{\left(\partial_{\eta}^{\alpha} b_{m^{\prime}-j / 2}\right)(y, \eta)}\left(\partial_{y}^{\alpha} \varphi_{\mu-\ell / 2}\right)(y, \eta ; x) .
$$

3) Let $\varphi^{*}\left(y, D_{y} ; x\right) \in \mathrm{OP}_{\mathrm{cl}}^{* \mu}$ be as before. Let $\psi\left(y, D_{y} ; x\right) \in \mathrm{OPH}_{\mathrm{cl}}^{\mu^{\prime}}$ with $\psi \sim \sum_{r \geq 0} \psi_{\mu^{\prime}-r / 2}$. Then

$$
\varphi^{*}\left(y, D_{y} ; x\right) \psi\left(y, D_{y} ; x\right)=b\left(y, D_{y}\right) \in \operatorname{OPS}_{\mathrm{cl}}^{\mu+\mu^{\prime}}\left(\mathbb{R}^{n-v}\right),
$$

with $b \sim \sum_{j \geq 0} b_{\mu+\mu^{\prime}-j / 2}$, where

$$
b_{\mu+\mu^{\prime}-j / 2}(y, \eta)=\sum_{2|\alpha|+\ell+r=j} \frac{1}{\alpha ! i^{|\alpha|}} \int \overline{\partial_{\eta}^{\alpha} \varphi_{\mu-\ell / 2}(y, \eta ; x)} \partial_{y}^{\alpha} \psi_{\mu^{\prime}-r / 2}(y, \eta ; x) d x .
$$
$C_{0}^{\infty}\left(\mathbb{R}^{n-v}\right)$ and $f \in C_{0}^{\infty}\left(\mathbb{R}^{n}\right)$, we have

$$
\left(\varphi\left(y, D_{y} ; x\right) g, f\right)_{L^{2}\left(\mathbb{R}^{n}\right)}=\left(g, \psi^{*}\left(y, D_{y} ; x\right) f\right)_{L^{2}\left(\mathbb{R}^{v}\right)},
$$

where $\psi^{*}\left(y, D_{y} ; x\right) \in \mathrm{OPH}_{\mathrm{cl}}^{* \mu}$ with $\psi \sim \sum_{j \geq 0} \psi_{\mu-j / 2}$ and

$$
\psi_{\mu-j / 2}(y, \eta ; x)=\sum_{2|\alpha|+\ell=j} \frac{i^{|\alpha|}}{\alpha !} \overline{\partial_{y}^{\alpha} \partial_{\eta}^{\alpha} \varphi_{\mu-\ell / 2}(y, \eta ; x)} .
$$


Point 3) in Lemma 3.12 takes care of a composition $\varphi^{*} \psi$. We need also to control $\psi \varphi^{*}$ and are, thus, forced to introduce the appropriate classes of symbols and operators (following Boutet de Monvel [3]).

\section{3. $\mathcal{H}^{m, k}$ classes}

Definition 3.13. For $m, k \in \mathbb{R}$, we put

$$
\mathcal{H}^{m, k}:=\bigcap_{j \geq 0} S^{m-j, k-2 j},
$$

and $\mathrm{OP} \mathcal{H}^{m, k}$ denotes the corresponding class of $\psi$ dos. Note that $\mathcal{H}^{m, k}=\mathcal{H}^{m-k / 2,0}$.

We need the corresponding homogeneous version.

DEFINITION 3.14. Let $m, k \in \mathbb{R}$. By $\mathcal{H}_{\mathrm{hom}}^{m, k}$, we denote the class of all smooth functions $a(y, \eta ; x, \xi)=a: T^{*} \mathbb{R}^{n-v} \backslash 0 \times T^{*} \mathbb{R}^{v} \longrightarrow \mathbb{C}$ such that

(i) $\quad a(y, \eta ;.) \in \mathcal{S}\left(\mathbb{R}_{(x, \xi)}^{2 v}\right)$ for all $(y, \eta) \in T^{*} \mathbb{R}^{n-v} \backslash 0$;

(ii) $a\left(y, t \eta ; t^{-1 / 2} x, t^{1 / 2} \xi\right)=t^{m-k / 2} a(y, \eta ; x, \xi), t>0$.

Note that $\mathcal{H}_{\mathrm{hom}}^{m, k}=\bigcap_{j \geq 0} S_{\mathrm{hom}}^{m-j, k-2 j}$ and that, furthermore, $\mathcal{H}_{\mathrm{hom}}^{m, k} \subset \mathcal{H}^{m, k}$ (by means of excision functions as before). By $\mathrm{OPH}_{\mathrm{cl}}^{m, k}$, we denote the class of $\psi \mathrm{dos}$ $a\left(z, D_{z}\right) \in \mathrm{OP} \mathcal{H}^{m, k}$ such that $a \sim \sum_{j \geq 0} a_{j / 2}^{(m, k)}(y, \eta ; x, \xi)$ with $a_{j / 2}^{(m, k)} \in \mathcal{H}_{\mathrm{hom}}^{m, k+j}$ and where the expansion means

$$
a-\sum_{j=0}^{N-1} a_{j / 2}^{(m, k)} \in \mathcal{H}^{m, k+N}, \quad \text { for all } N \geq 1 .
$$

We have the following lemma.

LEMMA 3.15.

1) Let $\varphi^{*}\left(y, D_{y} ; x\right) \in \mathrm{OPH}_{\mathrm{cl}}^{* \mu}, \psi\left(y, D_{y} ; x\right) \in \mathrm{OPH}_{\mathrm{cl}}^{\mu^{\prime}}$, with $\varphi \sim \sum_{\ell \geq 0} \varphi_{\mu-\ell / 2}$, $\psi \sim \sum_{r \geq 0} \psi_{\mu^{\prime}-r / 2}$. Then

$$
\psi\left(y, D_{y} ; x\right) \varphi^{*}\left(y, D_{y} ; x\right)=a\left(z, D_{z}\right) \in \mathrm{OP}_{\mathrm{cl}}^{\mu+\mu^{\prime}, 0},
$$

with $a \sim \sum_{j \geq 0} a_{j / 2}^{\left(\mu+\mu^{\prime}, 0\right)}$, where

$$
a_{j / 2}^{\left(\mu+\mu^{\prime}, 0\right)}(y, \eta ; x, \xi)=\sum_{2|\alpha|+\ell+r=j} \frac{e^{-i x \cdot \xi}}{\alpha ! i|\alpha|} \partial_{\eta}^{\alpha} \psi_{\mu^{\prime}-r / 2}(y, \eta ; x) \overline{\partial_{y}^{\alpha} \hat{\varphi}_{\mu-\ell / 2}(y, \eta ; \xi)} .
$$


2) Let $a\left(z, D_{z}\right) \in \mathrm{OPH}_{\mathrm{cl}}^{m, k}, \varphi\left(y, D_{y} ; x\right) \in \mathrm{OPH}_{\mathrm{cl}}^{\mu}$, with $a \sim \sum_{j \geq 0} a_{j / 2}^{(m, k)}$, $\varphi \sim \sum_{j \geq 0} \varphi_{\mu-j / 2}$. Then

$$
a\left(z, D_{z}\right) \varphi\left(y, D_{y} ; x\right)=\psi\left(y, D_{y} ; x\right) \in \mathrm{OPH}_{\mathrm{cl}}^{m-k / 2+\mu},
$$

with $\psi \sim \sum_{r \geq 0} \psi_{m-k / 2+\mu-r / 2}(y, \eta ; x)$ where

$$
\begin{aligned}
& \psi_{m-k / 2+\mu-r / 2}(y, \eta ; x) \\
& =\sum_{2|\alpha|+j+\ell=r} \frac{1}{\alpha ! i^{|\alpha|}}\left(\partial_{\eta}^{\alpha} a_{j / 2}^{(m, k)}\right)\left(y, \eta ; x, D_{x}\right)\left(\partial_{y}^{\alpha} \varphi_{\mu-\ell / 2}(y, \eta ; .)\right), \quad r \geq 0 .
\end{aligned}
$$

As usual, we omit the proof.

\subsection{Continuity properties and WF-relations}

In this subsection, we focus on the continuity properties in Sobolev spaces and the wave-front-set relations of the operators considered earlier.

First of all, we recall some standard notation (see [10, Vol. I]) concerning the wave-front-set relations.

Suppose we are given a linear continuous map $P: \mathcal{E}^{\prime}(X) \longrightarrow \mathcal{D}^{\prime}(Y)$, with $X \subset$ $\mathbb{R}_{x}^{n}, Y \subset \mathbb{R}_{y}^{m}$ given open sets (just to fix ideas) and let $\mathrm{k}_{P}(y, x)=\mathrm{k}_{P} \in \mathcal{D}^{\prime}(Y \times X)$ be the Schwartz kernel of $P$. We define

$$
\mathrm{WF}^{\prime}(P):=\left\{((y, \eta),(x, \xi)) \in\left(T^{*} Y \times T^{*} X\right) \backslash 0 ;(y, x, \eta,-\xi) \in \mathrm{WF}\left(\mathrm{k}_{P}\right)\right\} .
$$

With this notation, we have the following lemma.

LEMMA 3.16. One has the following facts.

1) $\quad A \in \mathrm{OPS}^{m, k} \Longrightarrow \mathrm{WF}^{\prime}(A) \subset \operatorname{diag}\left(T^{*} \mathbb{R}^{n} \backslash 0 \times T^{*} \mathbb{R}^{n} \backslash 0\right)$.

2) $A \in \mathrm{OPH}^{m, k} \Longrightarrow \mathrm{WF}^{\prime}(A) \subset \operatorname{diag}(\Sigma \times \Sigma)$.

3) $A \in \mathrm{OPH}_{\mathrm{cl}}^{\mu} \Longrightarrow \mathrm{WF}^{\prime}(A) \subset\{((x=0, y, \xi=0, \eta),(y, \eta)) ;(y, \eta) \in$ $\left.T^{*} \mathbb{R}^{n-v} \backslash 0\right\}$.

4) $A \in \mathrm{OPH}_{\mathrm{cl}}^{* \mu} \Longrightarrow \operatorname{WF}^{\prime}(A) \subset\{((y, \eta),(x=0, y, \xi=0, \eta)) ;(y, \eta) \in$ $\left.T^{*} \mathbb{R}^{n-v} \backslash 0\right\}$.

The proofs of 1) and 2) are already in [3]. Points 3) and 4) follow by direct inspection of the kernel $\mathrm{k}_{A}$.

As for the continuity in Sobolev spaces, we shall limit ourselves to what is strictly needed for the following lemma. 
LEMMA 3.17.

1) Let $A \in \mathrm{OPS}^{m,-k}, k \geq 0$, be properly supported. Then

$$
A: H_{\mathrm{loc}}^{t}\left(\mathbb{R}^{n}\right) \longrightarrow H_{\mathrm{loc}}^{t-m-k / 2}\left(\mathbb{R}^{n}\right)
$$

is continuous for all $t \in \mathbb{R}$.

2) Let $\mathrm{A} \in \mathrm{OP} \mathcal{H}^{m, k}$ be properly supported. Then

$$
A: H_{\mathrm{loc}}^{t}\left(\mathbb{R}^{n}\right) \longrightarrow H_{\mathrm{loc}}^{t-m+k / 2}\left(\mathbb{R}^{n}\right)
$$

is continuous for all $t \in \mathbb{R}$.

3) Let $A \in \mathrm{OPH}_{\mathrm{cl}}^{\mu}, B \in \mathrm{OPH}_{\mathrm{cl}}^{* \mu^{\prime}}$ be properly supported. Then

$$
A: H_{\mathrm{loc}}^{t}\left(\mathbb{R}^{n-v}\right) \longrightarrow H_{\mathrm{loc}}^{t-\mu}\left(\mathbb{R}^{n}\right), \quad B: H_{\mathrm{loc}}^{t}\left(\mathbb{R}^{n}\right) \longrightarrow H_{\mathrm{loc}}^{t-\mu^{\prime}}\left(\mathbb{R}^{n-v}\right),
$$

are continuous for all $t \in \mathbb{R}$.

Proof. 1) By Remark 3.2, if $k \geq 0$, then OPS ${ }^{m,-k} \subset \operatorname{OPS}_{1 / 2,1 / 2}^{m+k / 2}\left(\mathbb{R}^{n}\right)$. Hence, the result follows by the Calderón-Vaillancourt theorem.

2) The result is a consequence of point 1 ) since $\mathrm{OPH}^{m, k} \subset \mathrm{OPS}^{m-k / 2,0}$.

3) This part is actually a consequence of the general framework of Boutet de Monvel's paper [3]. However, in our particular setting, a more direct proof can be given by the standard procedure of considering in the first place symbols which do not depend on $y \in \mathbb{R}^{n-v}$ and then in the second place by perturbation. When considering symbols of the form $\varphi(\eta ; x)=\varphi \in \mathbf{H}_{\text {hom }}^{\mu}$, the continuity of $\varphi\left(D_{y} ; x\right): H^{t}\left(\mathbb{R}^{n-v}\right) \longrightarrow$ $H^{t-\mu}\left(\mathbb{R}^{n}\right)$ follows by observing that

$$
\int_{\mathbb{R}^{\nu}}|\chi(\eta) \hat{\varphi}(\eta ; \xi)|^{2} d \xi=|\eta|^{2 \mu} \int_{\mathbb{R}^{v}}\left|\chi(\eta) \hat{\varphi}\left(\frac{\eta}{|\eta|} ; \xi^{\prime}\right)\right|^{2} d \xi^{\prime} \leq C\left(1+|\eta|^{2}\right)^{\mu}
$$

$\chi$ being an excision function.

A final observation which will be crucial later on is the following.

LEMMA 3.18.

1) If $A \in \mathrm{OPS}^{m, \infty}$ and $B \in \mathrm{OPH}^{m^{\prime}, k^{\prime}}$ (properly supported), then both $A B$ and $B A$ are smoothing operators.

2) If $A \in \mathrm{OPS}^{m, \infty}, B \in \mathrm{OPH}^{* \mu}, C \in \mathrm{OPH}^{\mu^{\prime}}$ (properly supported), then $A C$ and $B A$ are smoothing operators. 
Proof. 1) For all $N \geq 0$, one has $A \in \mathrm{OPS}^{m, N}$, so that by a general result from Boutet de Monvel's [3] $A B, B A \in \mathrm{OP} \mathcal{H}^{m+m^{\prime}-k^{\prime} / 2-N / 2,0}$. It follows from Lemma 3.17, 2), that

$$
A B, B A: H_{\mathrm{loc}}^{t}\left(\mathbb{R}^{n}\right) \longrightarrow H_{\mathrm{loc}}^{t-m-m^{\prime}+k^{\prime} / 2+N / 2}\left(\mathbb{R}^{n}\right)
$$

are continuous, for all $t \in \mathbb{R}$. The conclusion follows by the arbitrariness of $N$.

2) Since $A \in \operatorname{OPS}^{m, N}$ for all $N \geq 0$, by [3, (5.11)], we have $B A \in$ $\bigcap_{N \geq 0} \mathrm{OPH}^{* \mu+m-N / 2}$, which proves that $B A$ is smoothing. The other case is similar. $\square$

\section{Parametrices}

As promised in the Introduction, given our initial operator $A$ for which $\operatorname{dim} \operatorname{Ker}\left(A_{\rho_{0}}\right)=d \geq 1$ and satisfying (H1), we want to construct a $d \times d$ system $\Lambda\left(y, D_{y}\right)$ of order $m-k / 2$, whose hypoellipticity at $\rho_{0}$ is equivalent to the hypoellipticity of $A$ at $\rho_{0}$. This construction will require the whole machinery developed in Section 3.

In order to simplify the exposition, we shall first make a 'global' construction, i.e. we suppose that $\tilde{U}=\mathbb{S}^{*} \Sigma=\mathbb{S}^{*} \mathbb{R}^{n-v}$ is admissible, so that, in particular, the vector-bundles $V_{1}$ and $V_{2}$ on $\Sigma$ are trivial.

We shall show afterwards how to microlocalize the construction.

We consider systems of the form

$$
\mathcal{A}=\left[\begin{array}{cc}
A & H^{-} \\
H^{+} & 0
\end{array}\right],
$$

where $A$ is our initial operator, $H^{-}=\left[H_{1}^{-} H_{2}^{-} \ldots H_{d}^{-}\right]$is a $1 \times d$ matrix of operators $H_{j}^{-} \in \mathrm{OPH}_{\mathrm{cl}}^{0}$ with

$$
\sigma\left(H_{j}^{-}\right) \sim \sum_{\ell \geq 0} \phi_{-\ell / 2}^{(j)}(y, \eta ; x), \quad 1 \leq j \leq d .
$$

We will always suppose that $\phi:=\left(\phi_{0}^{(1)}, \phi_{0}^{(2)}, \ldots, \phi_{0}^{(d)}\right)$ be a gauge of $V_{2}$. Furthermore,

$$
H^{+}=\left[\begin{array}{c}
H_{1}^{+} \\
\vdots \\
H_{d}^{+}
\end{array}\right]
$$

is a $d \times 1$ matrix of operators $H_{j}^{+} \in \mathrm{OPH}_{\mathrm{cl}}^{* 0}$ with

$$
\sigma\left(H_{j}^{+}\right) \sim \sum_{\ell \geq 0}{\phi^{\prime}}_{-\ell / 2}^{(j)}(y, \eta ; x), \quad 1 \leq j \leq d .
$$


We will always suppose that $\phi^{\prime}:=\left({\phi^{\prime}}_{0}^{(1)}, \phi_{0}^{\prime(2)}, \ldots, \phi_{0}^{\prime(d)}\right)$ be a gauge of $V_{1}$. Note that

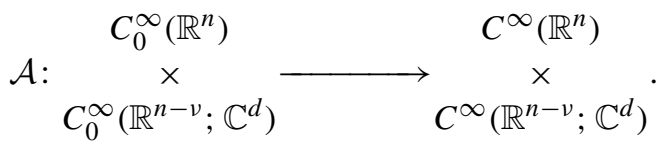

By definition, the localized operator associated with $\mathcal{A}$ at $\rho=(y, \eta) \in \Sigma$ is the system

$$
\mathcal{A}_{\rho, \phi, \phi^{\prime}}=\left[\begin{array}{cc}
A_{\rho} & h_{\phi}^{-}(\rho) \\
h_{\phi^{\prime}}^{+}(\rho) & 0
\end{array}\right] \text {. }
$$

The core of this paper is the following theorem.

THEOREM 4.1. Suppose (H1) holds and that $\mathbb{S}^{*} \mathbb{R}^{n-v}$ is admissible. Then, given any system $\mathcal{A}$ as in (45), there exist:

(i) $E \in \mathrm{OPS}_{\mathrm{cl}}^{-m,-k}$.

(ii) $K^{-}=\left[K_{1}^{-} \ldots K_{d}^{-}\right]$, a $1 \times d$ matrix of operators $K_{j}^{-} \in \mathrm{OPH}_{\mathrm{cl}}^{0}$ with $\sigma\left(K_{j}^{-}\right) \sim$ $\sum_{\ell \geq 0} \psi_{-\ell / 2}^{\prime(j)}(y, \eta ; x), 1 \leq j \leq d$, where $\psi_{0}^{\prime(j)}=\phi_{0}^{\prime(j)}, 1 \leq j \leq d$.

(iii) $K^{+}=\left[\begin{array}{c}K_{1}^{+} \\ \vdots \\ K_{d}^{+}\end{array}\right]$, a $d \times 1$ matrix of operators $K_{j}^{+} \in \mathrm{OPH}_{\mathrm{cl}}^{* 0}$, with $\sigma\left(K_{j}^{+}\right) \sim$ $\sum_{\ell \geq 0} \psi_{-\ell / 2}^{(j)}(y, \eta ; x), 1 \leq j \leq d$, where $\psi_{0}^{(j)}=\phi_{0}^{(j)}, 1 \leq j \leq d$.

(iv) A pseudodifferential $d \times d$ system $\Lambda=\Lambda\left(y, D_{y}\right)=\left(\Lambda^{(r j)}\left(y, D_{y}\right)\right)_{1 \leq r, j \leq d}$, whose entries $\Lambda^{(r j)} \in \mathrm{OPS}_{\mathrm{cl}}^{m-k / 2}\left(\mathbb{R}^{n-v}\right)$, with

$$
\sigma\left(\Lambda^{(r j)}\right) \sim \sum_{\ell \geq 0} \Lambda_{m-k / 2-\ell / 2}^{(r j)}(y, \eta)
$$

where

$$
\left(\Lambda_{m-k / 2}^{(r j)}(\rho)\right)_{1 \leq r, j \leq d}=\Lambda_{\phi, \phi^{\prime}}(\rho)=h_{\phi}^{+}(\rho) \circ A_{\rho} \circ h_{\phi^{\prime}}^{-}(\rho), \quad \text { for all } \rho \in \Sigma, \quad \text { (46) }
$$

such that the system

$$
\mathcal{E}=\left[\begin{array}{cc}
E & K^{-} \\
K^{+} & -\Lambda
\end{array}\right]
$$

is a two-sided parametrix of $\mathcal{A}$, i.e. $\mathcal{A} \mathcal{E}-\mathrm{id}$ and $\mathcal{E} \mathcal{A}-\mathrm{id}$ map $\mathcal{E}^{\prime}\left(\mathbb{R}^{n}\right) \times$ $\mathcal{E}^{\prime}\left(\mathbb{R}^{n-v} ; \mathbb{C}^{d}\right)$ into $C^{\infty}\left(\mathbb{R}^{n}\right) \times C^{\infty}\left(\mathbb{R}^{n-v} ; \mathbb{C}^{d}\right)$.

REMARK 4.2. The full expansion of the symbol of $\Lambda$ will be computed in the proof and put in evidence at the end. 
Proof. First step. Suppose we have already found a system $\mathcal{E}$ as in (47) such that

$$
\mathcal{A E}=\left[\begin{array}{cc}
\mathrm{id}+R & \text { smoothing } \\
\text { smoothing } & \mathrm{id}+\text { smoothing }
\end{array}\right],
$$

where $R \in \mathrm{OPS}^{0, \infty}$. Using the argument of [7] (see also [4]) gives the existence of $Q \in \mathrm{OPS}^{-m,-k}$ for which $A Q=\mathrm{id}+L, L \in \mathrm{OPH}^{0,0}$, so that $A Q R=R+L R$. It follows from Lemma 3.18 that $L R$ is smoothing. Hence,

$$
\left[\begin{array}{cc}
A & H^{-} \\
H^{+} & 0
\end{array}\right]\left[\begin{array}{cc}
E-Q R & K^{-} \\
K^{+} & -\Lambda
\end{array}\right]=\left[\begin{array}{cc}
\mathrm{id}+\text { smoothing } & \text { smoothing } \\
-H^{+} Q R+\text { smoothing } & \mathrm{id}+\text { smoothing }
\end{array}\right]
$$

Now, $E-Q R \in \mathrm{OPS}_{\mathrm{cl}}^{-m,-k}$ (since $Q R \in \mathrm{OPS}^{-m, \infty}$ ) and $H^{+} Q R$ is smoothing as a consequence of Lemma 3.18.

By denoting $E-Q R$ by $E$ again, we have a system of the form (47) which is a right parametrix of $\mathcal{A}$.

The same argument applies if we have already found a system $\mathcal{E}^{\prime}$ of the form (47) such that

$$
\mathcal{E}^{\prime} \mathcal{A}=\left[\begin{array}{cc}
\mathrm{id}+R^{\prime} & \text { smoothing } \\
\text { smoothing } & \mathrm{id}+\text { smoothing }
\end{array}\right],
$$

with $R^{\prime} \in \operatorname{OPS}^{0, \infty}$.

Once we have a right parametrix $\mathcal{E}$ and a left parametrix $\mathcal{E}^{\prime}$ of $\mathcal{A}$, we immediately conclude that $\mathcal{E}-\mathcal{E}^{\prime}=$ smoothing.

Second step. We now construct a system $\mathcal{E}$ satisfying (48). The construction of a system $\mathcal{E}^{\prime}$ satisfying (49) proceeds by the same arguments (e.g., by constructing a right parametrix for $\mathcal{A}^{*}$ ) and is left to the reader.

In order to carry out the construction, by Remark 3.5 we think of $A$ as an operator in $\mathrm{OPS}_{\mathrm{cl}}^{m, k}$. If $\sigma(A) \sim \sum_{r \geq 0} a_{m-r / 2}(z, \zeta)$ is the semi-regular asymptotic expansion of the symbol of $A$, with $a_{m-r / 2}$ vanishing at $\Sigma$ to order $k-r, 0 \leq r \leq k$, then, modulo $S^{m, \infty}$, we have

$$
\sigma(A) \sim \sum_{j \geq 0} \tilde{a}_{m-j / 2}(y, \eta ; x, \xi)
$$

where

$$
\tilde{a}_{m-j / 2}(y, \eta ; x, \xi)=\sum_{|\alpha|+|\beta|+r=k+j} \frac{1}{\alpha ! \beta !} \partial_{x}^{\alpha} \partial_{\xi}^{\beta} a_{m-r / 2}(0, y, 0, \eta) x^{\alpha} \xi^{\beta}
$$


belongs to $S_{\mathrm{hom}}^{m, k+j}$, for all $j \geq 0$. We put

$$
A_{\rho=(y, \eta)}^{(k+j)}:=\operatorname{Op}\left(\tilde{a}_{m-j / 2}\right)\left(y, \eta ; x, D_{x}\right) \in \operatorname{OPS}^{k+j}, \quad j \geq 0 .
$$

Note that $A_{\rho}^{(k)}$ is our localized operator $A_{\rho}$.

Since we look for $E \in \mathrm{OPS}_{\mathrm{cl}}^{-m,-k}$, we look for the asymptotic expansion (modulo $S^{-m, \infty}$ ) of its symbol

$$
\sigma(E) \sim \sum_{j \geq 0} \tilde{e}_{-m-j / 2}(y, \eta ; x, \xi), \quad \tilde{e}_{-m-j / 2} \in S_{\mathrm{hom}}^{-m,-k+j}, \quad j \geq 0 .
$$

In analogy with (50), we put

$$
E_{\rho=(y, \eta)}^{(-k+j)}:=\tilde{e}_{-m-j / 2}\left(y, \eta ; x, D_{x}\right) \in \mathrm{OPS}^{-k+j}, \quad j \geq 0 .
$$

Having already fixed the principal symbols of $K^{ \pm}$and $\Lambda$, by Theorem 2.13 we are forced to take

$$
E_{\rho}^{(-k)}:=E_{\rho}= \begin{cases}0 & \text { on } V_{2}(\rho) \\ \left(\left.A_{\rho}^{(k)}\right|_{V_{1}(\rho)^{\perp} \cap B^{k}\left(\mathbb{R}^{v}\right)}\right)^{-1} & \text { on } V_{2}(\rho)^{\perp}\end{cases}
$$

That $E_{\rho}^{(-k)} \in \mathrm{OPS}^{-k}$ with symbol $\tilde{e}_{-m}(y, \eta ; x, \xi) \in S_{\text {hom }}^{-m,-k}$ is a consequence of its very definition. We are, therefore, left with determining all the symbols

$$
\begin{gathered}
\tilde{e}_{-m-\ell / 2}, \quad \ell \geq 0 \\
\psi_{-\ell / 2}^{(j)}, \quad \psi_{-\ell / 2}^{(j)}, \quad 1 \leq j \leq d, \quad \ell \geq 0 \\
\Lambda_{m-k / 2-\ell / 2}^{(r j)}, \quad 1 \leq r, j \leq d, \quad \ell \geq 0
\end{gathered}
$$

Imposing (48) amounts to solving the following system of equations

1

2

3

4

$$
A E+\sum_{j=1}^{d} H_{j}^{-} K_{j}^{+}=\mathrm{id} \bmod \mathrm{OPS}^{0, \infty} \text {. }
$$$$
H_{j}^{+} E=\text { smoothing, } \quad 1 \leq j \leq d .
$$

$$
A K_{j}^{-}-\sum_{r=1}^{d} H_{r}^{-} \Lambda^{(r j)}=\text { smoothing, } \quad 1 \leq j \leq d .
$$

$$
H_{j}^{+} K_{r}^{-}-\delta_{j r}=\text { smoothing, } \quad 1 \leq j, r \leq d .
$$


In view of the composition rules recalled in Section 3, $\mathbf{1}$ through $\mathbf{4}$ are satisfied if and only if the following relations are fulfilled for each degree of homogeneity $\ell \geq 0$ :

$$
\begin{aligned}
& \mathbf{1}^{\prime} \quad \sum_{2|\alpha|+p+q=\ell} \frac{1}{\alpha ! i^{|\alpha|}}\left[\sigma_{(x, \xi)}\left(\partial_{\eta}^{\alpha} A_{(y, \eta)}^{(k+p)} \bullet \partial_{y}^{\alpha} E_{(y, \eta)}^{(-k+q)}\right)\right. \\
& \left.+\sum_{j=1}^{d} e^{-i x \cdot \xi} \partial_{\eta}^{\alpha} \phi_{-p / 2}^{(j)}(y, \eta ; x) \overline{\partial_{y}^{\alpha} \hat{\psi}_{-q / 2}^{(j)}(y, \eta ; \xi)}\right]= \begin{cases}1 & \text { if } \ell=0 \\
0 & \text { if } \ell \geq 1 .\end{cases} \\
& \mathbf{2}^{\prime} \quad \sum_{2|\alpha|+p+q=\ell} \frac{i^{|\alpha|} \overline{\alpha !}}{\left(\partial_{y}^{\alpha} E_{(y, \eta)}^{(-k+p)}\right)^{*} \partial_{\eta}^{\alpha} \phi_{-q / 2}^{\prime(j)}(y, \eta ; .)}=0, \quad 1 \leq j \leq d . \\
& \mathbf{3}^{\prime} \quad \sum_{2|\alpha|+p+q=\ell} \frac{1}{\alpha ! i^{|\alpha|}}\left[\partial_{\eta}^{\alpha} A_{(y, \eta)}^{(k+p)} \partial_{y}^{\alpha} \psi_{-q / 2}^{\prime(j)}(y, \eta ; .)\right. \\
& \left.-\sum_{r=1}^{d} \partial_{\eta}^{\alpha} \phi_{-p / 2}^{(r)}(y, \eta ; x) \partial_{y}^{\alpha} \Lambda_{m-k / 2-q / 2}^{(r j)}(y, \eta)\right]=0, \quad 1 \leq j \leq d . \\
& 4^{\prime} \quad \sum_{2|\alpha|+p+q=\ell} \frac{1}{\alpha ! i^{|\alpha|}}\left(\partial_{y}^{\alpha} \psi^{\prime(r)}-q / 2(y, \eta ; .), \partial_{\eta}^{\alpha}{\phi^{\prime}}_{-p / 2}^{(j)}(y, \eta ; .)\right)_{L^{2}\left(\mathbb{R}^{v}\right)}= \begin{cases}\delta_{j r} & \text { if } \ell=0, \\
0 & \text { if } \ell \geq 1 .\end{cases}
\end{aligned}
$$

By the very definition of $E_{(y, \eta)}^{(-k)}, \psi_{0}^{(j)}, \psi^{\prime(j)}, 1 \leq j \leq d$, and $\Lambda_{m-k / 2}(y, \eta)$, these relations are automatically satisfied when $\ell=0$, as we are going to show next.

- $\quad 4^{\prime}$ is just a reformulation of the gauge-orthonormality condition $h_{\phi^{\prime}}^{+}(\rho) \circ$ $h_{\phi^{\prime}}^{-}(\rho)=I_{\mathbb{C}^{d}}$, for all $\rho \in \Sigma$.

- $\quad 3^{\prime}$ reads as $A_{\rho}^{(k)} \psi_{0}^{\prime(j)}(\rho ;)=.\sum_{r=1}^{d} \Lambda_{m-k / 2}^{(r j)}(\rho) \phi_{0}^{(r)}(\rho$; .). Since, by definition,

$$
\Lambda_{m-k / 2}(\rho)=h_{\phi}^{+}(\rho) \circ A_{\rho}^{(k)} \circ h_{\phi^{\prime}}^{-}(\rho),
$$

and

$$
\begin{aligned}
A_{\rho}^{(k)}{\psi^{\prime}}_{0}^{(j)}(\rho ; .) & =A_{\rho}^{(k)} \phi_{0}^{\prime(j)}(\rho ; .) \\
& =\sum_{r=1}^{d}\left(A_{\rho}^{(k)} \phi_{0}^{\prime(j)}(\rho ; .), \phi_{0}^{(r)}(\rho ; .)\right)_{L^{2}\left(\mathbb{R}^{v}\right)} \phi_{0}^{(r)}(\rho ; .),
\end{aligned}
$$

$\mathbf{3}^{\prime}$ is obviously true.

- $\quad$ As regards $\mathbf{1}^{\prime}$, by the definition of $E_{\rho}^{(-k)}$ and the fact that $h_{\phi}^{+}(\rho) \circ h_{\phi}^{-}(\rho)=I_{\mathbb{C}^{d}}$, we have that, for all $f \in \mathcal{S}\left(\mathbb{R}^{v}\right)$,

$$
A_{\rho}^{(k)} \bullet E_{\rho}^{(-k)} f=f-\left(h_{\phi}^{-}(\rho) \circ h_{\phi}^{+}(\rho)\right) f=f-\pi_{2}(\rho) f, \quad \text { for all } \rho \in \Sigma .
$$


Now recall that the operator

$$
f \longmapsto\left(h_{\phi}^{-}(\rho) \circ h_{\phi}^{+}(\rho)\right) f=\sum_{j=1}^{d}\left(f, \phi_{0}^{(j)}(\rho ; .)\right)_{L^{2}\left(\mathbb{R}^{\nu}\right)} \phi_{0}^{(j)}(\rho ; .)
$$

has $(x, \xi)$-symbol (as a smoothing operator in the $x$-variables) precisely given by

$$
\sum_{j=1}^{d} e^{-i x \cdot \xi} \phi_{0}^{(j)}(\rho ; x) \overline{\hat{\phi}_{0}^{(j)}(\rho ; \xi)}
$$

which yields $\mathbf{1}^{\prime}$ for $\ell=0$.

- $\quad$ As regards $2^{\prime}$, we have to show that, for $1 \leq j \leq d, \phi_{0}^{\prime(j)}(\rho ;.) \in \operatorname{Ker}\left(\left(E_{\rho}^{(-k)}\right)^{*}\right)$, i.e. $\phi_{0}^{\prime(j)}(\rho ;.) \in \operatorname{Im}\left(E_{\rho}^{(-k)}\right)^{\perp}=V_{1}(\rho)$, which is true by hypothesis.

We next proceed in solving $\mathbf{1}^{\prime}-\mathbf{4}^{\prime}$ at step $\ell=1$ :

$$
\begin{gathered}
\mathbf{1}^{\prime} \sigma_{(x, \xi)}\left(A_{(y, \eta)}^{(k)} \bullet E_{(y, \eta)}^{(-k+1)}\right)+\sigma_{(x, \xi)}\left(A_{(y, \eta)}^{(k+1)} \bullet E_{(y, \eta)}^{(-k)}\right) \\
=-\sum_{j=1}^{d} e^{-i x \cdot \xi}\left[\phi_{-1 / 2}^{(j)}(y, \eta ; x) \overline{\left.\hat{\phi}_{0}^{(j)}(y, \eta ; \xi)\right]}\right. \\
\quad-\sum_{j=1}^{d} e^{-i x \cdot \xi}\left[\phi_{0}^{(j)}(y, \eta ; x) \overline{\left.\hat{\psi}_{-1 / 2}^{(j)}(y, \eta ; \xi)\right]} .\right. \\
\mathbf{2}^{\prime} \quad\left(E_{(y, \eta)}^{(-k+1)}\right)^{*} \phi_{0}^{\prime(j)}(y, \eta ; .)+\left(E_{(y, \eta)}^{(-k)}\right)^{*}{\phi^{\prime}}_{-1 / 2}^{(j)}(y, \eta ; .)=0, \quad 1 \leq j \leq d . \\
\mathbf{3}_{(y, \eta)}^{(k)} \psi_{-1 / 2}^{(j)}(y, \eta ; .)+A_{(y, \eta)}^{(k+1)} \phi_{0}^{\prime(j)}(y, \eta ; .) \\
=\sum_{r=1}^{d}\left[\phi_{0}^{(r)}(y, \eta ; x) \Lambda_{m-k / 2-1 / 2}^{(r j)}(y, \eta)+\phi_{-1 / 2}^{(r)}(y, \eta ; x) \Lambda_{m-k / 2}^{(r j)}(y, \eta)\right], \\
\text { for } 1 \leq j \leq d . \\
\quad\left(\psi_{-1 / 2}^{(r)}(y, \eta ; .), \phi_{0}^{\prime(j)}(y, \eta ; .)\right)_{L^{2}\left(\mathbb{R}^{v}\right)}+\left(\phi_{0}^{\prime(r)}(y, \eta ; .), \phi_{-1 / 2}^{\prime(j)}(y, \eta ; .)\right)_{L^{2}\left(\mathbb{R}^{v}\right)}=0, \\
\text { for } 1 \leq j, r \leq d .
\end{gathered}
$$

We first solve $\mathbf{3}^{\prime}$ and $\mathbf{4}^{\prime}$ for $\psi_{-1 / 2}^{\prime(j)}$ and $\Lambda_{m-k / 2-1 / 2}^{(r j)}$. We look for $\psi_{-1 / 2}^{\prime(j)}$ in the form

$$
\psi_{-1 / 2}^{\prime(j)}(y, \eta ; x)=\psi_{-1 / 2,1}^{\prime(j)}(y, \eta ; x)+\psi_{-1 / 2,2}^{\prime(j)}(y, \eta ; x),
$$

where $\psi_{-1 / 2,1}^{\prime(j)}(y, \eta ;.) \in V_{1}(y, \eta)$ and $\psi_{-1 / 2,2}^{\prime(j)}(y, \eta ;.) \in V_{1}(y, \eta)^{\perp}$. From $4^{\prime}$, we get

$$
\left(\psi_{-1 / 2,1}^{\prime(r)}(y, \eta ; .), \phi_{0}^{\prime(j)}(y, \eta ; .)\right)_{L^{2}\left(\mathbb{R}^{v}\right)}=-\left(\phi_{0}^{\prime(r)}(y, \eta ; .), \phi_{-1 / 2}^{\prime(j)}(y, \eta ; .)\right)_{L^{2}\left(\mathbb{R}^{v}\right)},
$$


for all $j, r=1, \ldots, d$, which determines uniquely the components $\psi_{-1 / 2,1}^{\prime(j)}, j=$ $1, \ldots, d$.

Plugging (52) into $\mathbf{3}^{\prime}$ yields

$$
\begin{aligned}
A_{(y, \eta)}^{(k)} \psi_{-1 / 2,2}^{\prime(j)}(y, \eta ; .)=\sum_{r=1}^{d} \phi_{0}^{(r)}(y, \eta ; x) \Lambda_{m-k / 2-1 / 2}^{(r j)}(y, \eta) \\
\quad-A_{(y, \eta)}^{(k)} \psi_{-1 / 2,1}^{(j)}(y, \eta ; x)+\sum_{r=1}^{d} \phi_{-1 / 2}^{(r)}(y, \eta ; x) \Lambda_{m-k / 2}^{(r j)}(y, \eta) \\
-A_{(y, \eta)}^{(k+1)} \phi_{0}^{\prime(j)}(y, \eta ; .)=: F_{j}(y, \eta ; x), \quad 1 \leq j \leq d .
\end{aligned}
$$

The obvious idea is to look for $\Lambda_{m-k / 2-1 / 2}^{(r j)}(y, \eta)$ such that $F_{j}(y, \eta ;.) \in V_{2}(y, \eta)^{\perp}$. Taking the $L^{2}\left(\mathbb{R}^{v}\right)$-inner product of $F_{j}(y, \eta ;$.$) with the functions \phi_{0}^{(s)}(y, \eta ;),. 1 \leq$ $s \leq d$, and using the identity $h_{\phi}^{+}(y, \eta) \circ h_{\phi}^{-}(y, \eta)=I_{\mathbb{C}^{d}}$, we obtain

$$
\begin{aligned}
\Lambda_{m-k / 2-1 / 2}^{(r j)}(y, \eta)= & \left(A_{(y, \eta)}^{(k)} \psi_{-1 / 2,1}^{(j)}(y, \eta ; .), \phi_{0}^{(r)}(y, \eta ; .)\right)_{L^{2}\left(\mathbb{R}^{v}\right)} \\
& +\left(A_{(y, \eta)}^{(k+1)} \phi_{0}^{\prime(j)}(y, \eta ; .), \phi_{0}^{(r)}(y, \eta ; .)\right)_{L^{2}\left(\mathbb{R}^{v}\right)} \\
& -\sum_{s=1}^{d} \Lambda_{m-k / 2}^{(s j)}(y, \eta)\left(\phi_{-1 / 2}^{(s)}(y, \eta ; .), \phi_{0}^{(r)}(y, \eta ; .)\right)_{L^{2}\left(\mathbb{R}^{v}\right)}
\end{aligned}
$$

Since $A_{(y, \eta)}^{(k)}: V_{1}(y, \eta)^{\perp} \cap B^{k}\left(\mathbb{R}^{v}\right) \stackrel{\sim}{\longrightarrow} V_{2}(y, \eta)^{\perp}$, we take

$$
\psi_{-1 / 2,2}^{\prime(j)}(y, \eta ; .)=E_{(y, \eta)}^{(-k)} F_{j}(y, \eta ; .), \quad 1 \leq j \leq d .
$$

We now turn to $\mathbf{1}^{\prime}$ and $\mathbf{2}^{\prime}$. Define

$$
\begin{aligned}
& R_{(y, \eta)}^{(1)}: \mathcal{S}\left(\mathbb{R}^{v}\right) \ni f \longmapsto \sum_{j=1}^{d}\left(f, \psi_{-1 / 2}^{(j)}(y, \eta ; .)\right)_{L^{2}\left(\mathbb{R}^{v)}\right.} \phi_{0}^{(j)}(y, \eta ; .) \in \mathcal{S}\left(\mathbb{R}^{v}\right), \\
& S_{(y, \eta)}^{(1)}: \mathcal{S}\left(\mathbb{R}^{v}\right) \ni f \longmapsto \sum_{j=1}^{d}\left(f, \phi_{0}^{(j)}(y, \eta ; .)\right)_{L^{2}\left(\mathbb{R}^{v}\right)} \phi_{-1 / 2}^{(j)}(y, \eta ; .) \in \mathcal{S}\left(\mathbb{R}^{v}\right), \\
& \pi_{2}(y, \eta): \mathcal{S}\left(\mathbb{R}^{v}\right) \ni f \longmapsto \sum_{j=1}^{d}\left(f, \phi_{0}^{(j)}(y, \eta ; .)\right)_{L^{2}\left(\mathbb{R}^{v}\right)} \phi_{0}^{(j)}(y, \eta ; .) \in \mathcal{S}\left(\mathbb{R}^{v}\right) .
\end{aligned}
$$

Hence $\mathbf{1}^{\prime}$ reduces to the operator equation

$$
A_{(y, \eta)}^{(k)} \bullet E_{(y, \eta)}^{(-k+1)}=-\left(A_{(y, \eta)}^{(k+1)} \bullet E_{(y, \eta)}^{(-k)}+S_{(y, \eta)}^{(1)}\right)-R_{(y, \eta)}^{(1)}=:-T_{(y, \eta)}^{(1)}-R_{(y, \eta)}^{(1)},
$$


with unknowns $E_{(y, \eta)}^{(-k+1)}$ and $R_{(y, \eta)}^{(1)}$. Recall that, by the very definition,

$$
A_{(y, \eta)}^{(k)} \bullet E_{(y, \eta)}^{(-k)}=\mathrm{id}-\pi_{2}(y, \eta)
$$

(and that $\pi_{2}(y, \eta)^{2}=\pi_{2}(y, \eta)$ since $\left.h_{\phi}^{+}(y, \eta) \circ h_{\phi}^{-}(y, \eta)=I_{\mathbb{C}^{d}}\right)$. We take as $E_{(y, \eta)}^{(-k+1)}$ the operator defined by

$$
\begin{aligned}
E_{(y, \eta)}^{(-k+1)} & : \mathcal{S}\left(\mathbb{R}^{v}\right) \ni f \longmapsto-E_{(y, \eta)}^{(-k)} \bullet\left(\mathrm{id}-\pi_{2}(y, \eta)\right) \bullet T_{(y, \eta)}^{(1)} f \\
& +\sum_{j=1}^{d}\left(f, \gamma_{-1 / 2}^{(j)}(y, \eta ; .)\right)_{L^{2}\left(\mathbb{R}^{\nu}\right)} \phi_{0}^{\prime(j)}(y, \eta ; .) \in \mathcal{S}\left(\mathbb{R}^{\nu}\right)
\end{aligned}
$$

for some $\gamma_{-1 / 2}^{(j)} \in \mathbf{H}_{\mathrm{hom}}^{-1 / 2}$ to be determined. Plugging (62) into (60) gives

$$
\begin{aligned}
A_{(y, \eta)}^{(k)} \bullet E_{(y, \eta)}^{(-k+1)} f= & -\left(\operatorname{id}-\pi_{2}(y, \eta)\right) \bullet T_{(y, \eta)}^{(1)} f \\
& +\sum_{j=1}^{d}\left(f, \gamma_{-1 / 2}^{(j)}(y, \eta ; .)\right)_{L^{2}\left(\mathbb{R}^{v}\right)} A_{(y, \eta)}^{(k)} \phi_{0}^{\prime(j)}(y, \eta ; .) \\
= & -T_{(y, \eta)}^{(1)} f-R_{(y, \eta)}^{(1)} f
\end{aligned}
$$

whence we must have

$$
\pi_{2}(y, \eta) \bullet T_{(y, \eta)}^{(1)} f+\sum_{j=1}^{d}\left(f, \gamma_{-1 / 2}^{(j)}(y, \eta ; .)\right)_{L^{2}\left(\mathbb{R}^{v}\right)} A_{(y, \eta)}^{(k)} \phi_{0}^{\prime(j)}(y, \eta ; .)+R_{(y, \eta)}^{(1)} f=0
$$

for all $f \in \mathcal{S}\left(\mathbb{R}^{v}\right)$, that is to say

$$
\begin{aligned}
& \sum_{j=1}^{d}\left(f,\left(T_{(y, \eta)}^{(1)}\right)^{*} \phi_{0}^{(j)}(y, \eta ; .)+\psi_{-1 / 2}^{(j)}(y, \eta ; .)\right)_{L^{2}\left(\mathbb{R}^{\nu}\right)} \phi_{0}^{(j)}(y, \eta ; .) \\
& +\sum_{j, r=1}^{d}\left(f, \overline{\left(A_{(y, \eta)}^{(k)} \phi_{0}^{\prime(r)}(y, \eta ; .), \phi_{0}^{(j)}(y, \eta ; .)\right)}{ }_{L^{2}\left(\mathbb{R}^{\nu}\right)} \gamma_{-1 / 2}^{(r)}(y, \eta ; .)\right)_{L^{2}\left(\mathbb{R}^{\nu}\right)} \phi_{0}^{(j)}(y, \eta ; .)=0,
\end{aligned}
$$

for all $f \in \mathcal{S}\left(\mathbb{R}^{v}\right)$. As a consequence, we obtain

$$
\begin{aligned}
\psi_{-1 / 2}^{(j)}(y, \eta ; x)= & -\left[\left(T_{(y, \eta)}^{(1)}\right)^{*} \phi_{0}^{(j)}(y, \eta ; .)\right. \\
& \left.+\sum_{r=1}^{d} \overline{\left(A_{(y, \eta)}^{(k)}{\phi^{\prime}}_{0}^{(r)}(y, \eta ; .), \phi_{0}^{(j)}(y, \eta ; .)\right)} L_{L^{2}\left(\mathbb{R}^{v}\right)} \gamma_{-1 / 2}^{(r)}(y, \eta ; x)\right] \\
& 1 \leq j \leq d
\end{aligned}
$$


It remains to pick the $\gamma_{-1 / 2}^{(r)}, 1 \leq r \leq d$. For this purpose, we finally use $\mathbf{2}^{\prime}$, i.e.

$$
\left(\phi_{0}^{\prime(j)}(y, \eta ; .), E_{(y, \eta)}^{(-k+1)} f\right)_{L^{2}\left(\mathbb{R}^{v}\right)}+\left(\phi_{-1 / 2}^{\prime(j)}(y, \eta ; .), E_{(y, \eta)}^{(-k)} f\right)_{L^{2}\left(\mathbb{R}^{v}\right)}=0,
$$

for all $f \in \mathcal{S}\left(\mathbb{R}^{v}\right)$ and all $j=1, \ldots, d$, i.e. using (62),

$$
\begin{aligned}
& \left(\phi_{0}^{\prime(j)}(y, \eta ; .),-E_{(y, \eta)}^{(-k)} \bullet\left(\mathrm{id}-\pi_{2}(y, \eta)\right) \bullet T_{(y, \eta)}^{(1)} f\right. \\
& \left.\quad+\sum_{r=1}^{d}\left(f, \gamma_{-1 / 2}^{(r)}(y, \eta ; .)\right)_{L^{2}\left(\mathbb{R}^{v}\right)} \phi_{0}^{\prime(r)}(y, \eta ; .)\right)_{L^{2}\left(\mathbb{R}^{v}\right)} \\
& \quad+\left(\phi_{-1 / 2}^{\prime(j)}(y, \eta ; .), E_{(y, \eta)}^{(-k)} f\right)_{L^{2}\left(\mathbb{R}^{v}\right)}=0
\end{aligned}
$$

for all $f \in \mathcal{S}\left(\mathbb{R}^{v}\right)$ and all $j=1, \ldots, d$, which can be rewritten as

$$
\begin{gathered}
\left(f,\left(E_{(y, \eta)}^{(-k)}\right)^{*} \phi_{-1 / 2}^{\prime(j)}(y, \eta ; .)-\left[E_{(y, \eta)}^{(-k)} \bullet\left(\operatorname{id}-\pi_{2}(y, \eta)\right) \bullet T_{(y, \eta)}^{(1)}\right]^{*} \phi_{0}^{\prime(j)}(y, \eta ; .)\right. \\
\left.+\gamma_{-1 / 2}^{(j)}(y, \eta ; .)\right)_{L^{2}\left(\mathbb{R}^{v}\right)}=0, \quad \text { for all } f \in \mathcal{S}\left(\mathbb{R}^{v}\right)
\end{gathered}
$$

which determines the $\gamma_{-1 / 2}^{(j)}$ uniquely. This completes step $\ell=1$.

An important observation at this point is the following.

REMARK 4.3. Suppose that the following parity assumption is satisfied:

(H2). The gauge $\phi=\left(\phi_{1}, \ldots, \phi_{d}\right)$ of $V_{2}$ and the gauge $\phi^{\prime}=\left(\phi_{1}^{\prime}, \ldots, \phi_{d}^{\prime}\right)$ of $V_{1}$ have the same parity in $x$, that is, for every $(y, \eta)$ the functions $\phi_{j}(y, \eta ;),. \phi_{r}^{\prime}(y, \eta ;$.$) ,$ $1 \leq j, r \leq d$, are either all even or all odd in $x$.

Since $k$ is even (see point 3) of Proposition 2.1), $A_{(y, \eta)}^{(k+1)}$ flips the parity, so that all the terms $\left(A_{(y, \eta)}^{(k+1)} \phi_{0}^{\prime(j)}(y, \eta ; .), \phi_{0}^{(r)}(y, \eta ; .)\right)_{L^{2}\left(\mathbb{R}^{\nu}\right)}$ in (55) vanish. Furthermore, if the terms $\phi_{-1 / 2}$ and $\phi_{-1 / 2}^{\prime}$, respectively, in the asymptotic expansion of the symbols of the operators $\mathrm{H}^{-}$and $\mathrm{H}^{+}$, respectively, are chosen to be zero, it turns out that $\Lambda_{m-k / 2-1 / 2}^{(r j)}(y, \eta)=0$ for all $(y, \eta)$ and all $1 \leq j, r \leq d$.

We now consider equations $\mathbf{1}^{\prime}-\mathbf{4}^{\prime}$ at step $\ell=2$. It will then be clear how to proceed by induction and this will be left to the reader. To simplify notation, expressions of the form $\sum_{|\alpha|=1} \partial_{\eta}^{\alpha} F \partial_{y}^{\alpha} G$ will be shortened to $\partial_{\eta} F \partial_{y} G$. Also, equation $\mathbf{1}^{\prime}$ will be written in operator form. We hence have:

$$
\begin{aligned}
\mathbf{1}^{\prime} A_{(y, \eta)}^{(k)} \bullet & E_{(y, \eta)}^{(-k+2)} f \\
= & -\left[A_{(y, \eta)}^{(k+1)} \bullet E_{(y, \eta)}^{(-k+1)}+A_{(y, \eta)}^{(k+2)} \bullet E_{(y, \eta)}^{(-k)}+\frac{1}{i} \partial_{\eta} A_{(y, \eta)}^{(k)} \bullet \partial_{y} E_{(y, \eta)}^{(-k)}+S_{(y, \eta)}^{(2)}\right] f \\
& -R_{(y, \eta)}^{(2)} f=:-T_{(y, \eta)}^{(2)} f-R_{(y, \eta)}^{(2)} f, \quad \text { for all } f \in \mathcal{S}\left(\mathbb{R}^{v}\right),
\end{aligned}
$$


where

$$
\begin{aligned}
& R_{(y, \eta)}^{(2)} f=\sum_{j=1}^{d}\left(f, \psi_{-1}^{(j)}(y, \eta ; .)\right)_{L^{2}\left(\mathbb{R}^{\nu}\right)} \phi_{0}^{(j)}(y, \eta ; .), \\
& S_{(y, \eta)}^{(2)} f=\sum_{j=1}^{d}\left[\left(f, \psi_{-1 / 2}^{(j)}(y, \eta ; .)\right)_{L^{2}\left(\mathbb{R}^{\nu}\right)} \phi_{-1 / 2}^{(j)}(y, \eta ; .)\right. \\
& +\left(f, \phi_{0}^{(j)}(y, \eta ; .)\right)_{L^{2}\left(\mathbb{R}^{v}\right)} \phi_{-1}^{(j)}(y, \eta ; .) \\
& \left.+\frac{1}{i}\left(f, \partial_{y} \phi_{0}^{(j)}(y, \eta ; .)\right)_{L^{2}\left(\mathbb{R}^{v}\right)} \partial_{\eta} \phi_{0}^{(j)}(y, \eta ; .)\right] \\
& \mathbf{2}^{\prime}\left(E_{(y, \eta)}^{(-k+2)}\right)^{*} \phi_{0}^{\prime(j)}(y, \eta ; .)+\left(E_{(y, \eta)}^{(-k+1)}\right)^{*} \phi_{-1 / 2}^{\prime(j)}(y, \eta ; .) \\
& +\left(E_{(y, \eta)}^{(-k)}\right)^{*} \phi_{-1}^{\prime(j)}(y, \eta ; .)+\frac{1}{i}\left(\partial_{y} E_{(y, \eta)}^{(-k)}\right)^{*} \partial_{\eta} \phi_{0}^{\prime(j)}(y, \eta ; .)=0, \quad 1 \leq j \leq d . \\
& \mathbf{3}^{\prime} A_{(y, \eta)}^{(k)} \psi_{-1}^{\prime(j)}(y, \eta ; .)+A_{(y, \eta)}^{(k+1)} \psi_{-1 / 2}^{\prime(j)}(y, \eta ; .) \\
& +A_{(y, \eta)}^{(k+2)} \phi_{0}^{\prime(j)}(y, \eta ; .)+\frac{1}{i} \partial_{\eta} A_{(y, \eta)}^{(k)} \partial_{y} \phi_{0}^{\prime(j)}(y, \eta ; .) \\
& =\sum_{r=1}^{d}\left[\phi_{0}^{(r)}(y, \eta ; x) \Lambda_{m-k / 2-1}^{(r j)}(y, \eta)\right. \\
& +\phi_{-1 / 2}^{(r)}(y, \eta ; x) \Lambda_{m-k / 2-1 / 2}^{(r j)}(y, \eta)+\phi_{-1}^{(r)}(y, \eta ; x) \Lambda_{m-k / 2}^{(r j)}(y, \eta) \\
& \left.+\frac{1}{i} \partial_{\eta} \phi_{0}^{(r)}(y, \eta ; x) \partial_{y} \Lambda_{m-k / 2}^{(r j)}(y, \eta)\right], \quad \text { for } 1 \leq j \leq d . \\
& \mathbf{4}^{\prime}\left(\psi_{-1}^{\prime(r)}(y, \eta ; .), \phi_{0}^{\prime(j)}(y, \eta ; .)\right)_{L^{2}\left(\mathbb{R}^{v}\right)}+\left(\psi_{-1 / 2}^{\prime(r)}(y, \eta ; .), \phi_{-1 / 2}^{\prime(j)}(y, \eta ; .)\right)_{L^{2}\left(\mathbb{R}^{v}\right)} \\
& +\left(\phi_{0}^{\prime(r)}(y, \eta ; .), \phi_{-1}^{\prime(j)}(y, \eta ; .)\right)_{L^{2}\left(\mathbb{R}^{v}\right)} \\
& +\frac{1}{i}\left(\partial_{y} \phi_{0}^{\prime(r)}(y, \eta ; .), \partial_{\eta} \phi_{0}^{\prime(j)}(y, \eta ; .)\right)_{L^{2}\left(\mathbb{R}^{v}\right)}=0, \quad \text { for all } 1 \leq j, r \leq d .
\end{aligned}
$$

As before, we begin by solving $\mathbf{3}^{\prime}$ and $\mathbf{4}^{\prime}$ for $\psi_{-1}^{\prime(j)}$ and $\Lambda_{m-k / 2-1}^{(r j)}$. We look for $\psi_{-1}^{\prime(j)}$ in the form

$$
\psi_{-1}^{\prime(j)}(y, \eta ; x)=\psi_{-1,1}^{\prime(j)}(y, \eta ; x)+\psi_{-1,2}^{\prime(j)}(y, \eta ; x),
$$

where $\psi_{-1,1}^{\prime(j)}(y, \eta ;.) \in V_{1}(y, \eta)$ and $\psi_{-1,2}^{\prime(j)}(y, \eta ;.) \in V_{1}(y, \eta)^{\perp}$. From $\mathbf{4}^{\prime}$, we get

$$
\left(\psi_{-1,1}^{\prime(r)}(y, \eta ; .), \phi_{0}^{\prime(j)}(y, \eta ; .)\right)_{L^{2}\left(\mathbb{R}^{v}\right)}=\text { given function, }
$$

for all $j, r=1, \ldots, d$, which determines the components $\psi_{-1,1}^{\prime(j)}, j=1, \ldots, d$, uniquely. 
Plugging (70) into $\mathbf{3}^{\prime}$ yields

$$
\begin{aligned}
A_{(y, \eta)}^{(k)} \psi_{-1,2}^{\prime(j)}(y, \eta ; .)= & \sum_{r=1}^{d} \phi_{0}^{(r)}(y, \eta ; x) \Lambda_{m-k / 2-1}^{(r j)}(y, \eta) \\
& -A_{(y, \eta)}^{(k)} \psi_{-1,1}^{\prime(j)}(y, \eta ; x)+G_{j}(y, \eta ; x) \\
= & : F_{j}(y, \eta ; x), \quad 1 \leq j \leq d,
\end{aligned}
$$

where the $G_{j}$ are given. Once again we look for $\Lambda_{m-k / 2-1}^{(r j)}(y, \eta)$ such that $F_{j}(y, \eta ;.) \in V_{2}(y, \eta)^{\perp}$. Taking the $L^{2}\left(\mathbb{R}^{v}\right)$-inner product of $F_{j}(y, \eta ;$.$) with the$ functions $\phi_{0}^{(s)}(y, \eta ;),. 1 \leq s \leq d$, yields

$$
\begin{aligned}
\Lambda_{m-k / 2-1}^{(r j)}(y, \eta)= & \left(A_{(y, \eta)}^{(k)} \psi_{-1,1}^{\prime(j)}(y, \eta ; .), \phi_{0}^{(r)}(y, \eta ; .)\right)_{L^{2}\left(\mathbb{R}^{v}\right)} \\
& -\left(G_{j}(y, \eta ; .), \phi_{0}^{(r)}(y, \eta ; .)\right)_{L^{2}\left(\mathbb{R}^{v}\right)}
\end{aligned}
$$

for all $1 \leq j, r \leq d$, and, as a consequence, we also get

$$
\psi_{-1,2}^{\prime(j)}(y, \eta ; x)=E_{(y, \eta)}^{(-k)} F_{j}(y, \eta ; .), \quad 1 \leq j \leq d .
$$

To solve $\mathbf{1}^{\prime}$ and $\mathbf{2}^{\prime}$, we take as $E_{(y, \eta)}^{(-k+2)}$ the operator defined by

$$
\begin{gathered}
E_{(y, \eta)}^{(-k+2)}: \mathcal{S}\left(\mathbb{R}^{\nu}\right) \ni f \longmapsto-E_{(y, \eta)}^{(-k)} \bullet\left(\mathrm{id}-\pi_{2}(y, \eta)\right) \bullet T_{(y, \eta)}^{(2)} f \\
+\sum_{j=1}^{d}\left(f, \gamma_{-1}^{(j)}(y, \eta ; .)\right)_{L^{2}\left(\mathbb{R}^{v}\right)} \phi_{0}^{\prime(j)}(y, \eta ; .) \in \mathcal{S}\left(\mathbb{R}^{v}\right)
\end{gathered}
$$

for some $\gamma_{-1}^{(j)} \in \mathbf{H}_{\text {hom }}^{-1}$ to be determined. Plugging (75) into $\mathbf{1}^{\prime}$ yields

$$
\begin{aligned}
A_{(y, \eta)}^{(k)} \bullet E_{(y, \eta)}^{(-k+2)} f= & -\left(\mathrm{id}-\pi_{2}(y, \eta)\right) \bullet T_{(y, \eta)}^{(2)} f \\
& +\sum_{j=1}^{d}\left(f, \gamma_{-1}^{(j)}(y, \eta ; .)\right)_{L^{2}\left(\mathbb{R}^{v}\right)} A_{(y, \eta)}^{(k)} \phi_{0}^{\prime(j)}(y, \eta ; .) \\
= & -T_{(y, \eta)}^{(2)} f-R_{(y, \eta)}^{(2)} f,
\end{aligned}
$$

whence we must have

$$
\pi_{2}(y, \eta) \bullet T_{(y, \eta)}^{(2)} f+\sum_{j=1}^{d}\left(f, \gamma_{-1}^{(j)}(y, \eta ; .)\right)_{L^{2}\left(\mathbb{R}^{\nu}\right)} A_{(y, \eta)}^{(k)} \phi_{0}^{\prime(j)}(y, \eta ; .)+R_{(y, \eta)}^{(2)} f=0
$$


for all $f \in \mathcal{S}\left(\mathbb{R}^{v}\right)$, that is to say

$$
\begin{aligned}
\sum_{j=1}^{d}(f, & \left.\left(T_{(y, \eta)}^{(2)}\right)^{*} \phi_{0}^{(j)}(y, \eta ; .)+\psi_{-1}^{(j)}(y, \eta ; .)\right)_{L^{2}\left(\mathbb{R}^{v}\right)} \phi_{0}^{(j)}(y, \eta ; .) \\
& +\sum_{j=1}^{d}\left(f, \gamma_{-1}^{(j)}(y, \eta ; .)\right)_{L^{2}\left(\mathbb{R}^{v}\right)} A_{(y, \eta)}^{(k)}{\phi^{\prime}}_{0}^{(j)}(y, \eta ; .)=0, \quad \text { for all } f \in \mathcal{S}\left(\mathbb{R}^{v}\right) .
\end{aligned}
$$

As before, we obtain

$$
\begin{aligned}
\psi_{-1}^{(j)}(y, \eta ; x)= & -\left[\left(T_{(y, \eta)}^{(2)}\right)^{*} \phi_{0}^{(j)}(y, \eta ; .)\right. \\
& \left.+\sum_{r=1}^{d} \overline{\left(A_{(y, \eta)}^{(k)} \phi_{0}^{\prime(r)}(y, \eta ; .), \phi_{0}^{(j)}(y, \eta ; .)\right)}{ }_{L^{2}\left(\mathbb{R}^{v}\right)} \gamma_{-1}^{(r)}(y, \eta ; x)\right] \\
& 1 \leq j \leq d
\end{aligned}
$$

It remains to pick the $\gamma_{-1}^{(r)}, 1 \leq r \leq d$. For this purpose, we finally use $\mathbf{2}^{\prime}$, i.e.,

$$
\left(\phi_{0}^{\prime(j)}(y, \eta ; .), E_{(y, \eta)}^{(-k+2)} f\right)_{L^{2}\left(\mathbb{R}^{v}\right)}+\left(L_{j}(y, \eta ; .), f\right)_{L^{2}\left(\mathbb{R}^{v}\right)}=0,
$$

for all $f \in \mathcal{S}\left(\mathbb{R}^{v}\right)$ and all $j=1, \ldots, d$, where the functions $L_{j}(y, \eta ;$.$) are given. As$ before, we get

$$
\begin{aligned}
\left(f, L_{j}(y, \eta ; .)-\right. & {\left[E_{(y, \eta)}^{(-k)} \bullet\left(\mathrm{id}-\pi_{2}(y, \eta)\right) \bullet T_{(y, \eta)}^{(2)}\right]^{*} \phi_{0}^{\prime(j)}(y, \eta ; .) } \\
+ & \left.\gamma_{-1}^{(j)}(y, \eta ; .)\right)_{L^{2}\left(\mathbb{R}^{v}\right)}=0, \quad \text { for all } f \in \mathcal{S}\left(\mathbb{R}^{v}\right),
\end{aligned}
$$

which determines the $\gamma_{-1}^{(j)}$ uniquely This completes the proof of the theorem.

From the above proof, we may now, as promised, spell out the matrices $\Lambda_{m-k / 2-\ell / 2}^{(r j)}(y, \eta), 1 \leq r, j \leq d, \ell \geq 0$, in the asymptotic expansion of the symbol of $\Lambda\left(y, D_{y}\right)$.

As regards the principal symbol we have

$$
\Lambda_{m-k / 2}^{(r j)}(y, \eta)=\left(A_{(y, \eta)}^{(k)} \phi_{0}^{\prime(j)}(y, \eta ; .), \phi_{0}^{(r)}(y, \eta ; .)\right)_{L^{2}\left(\mathbb{R}^{\nu}\right)} .
$$

For the lower-order terms $\Lambda_{m-k / 2-\ell / 2}^{(r j)}, \ell \geq 1$, on supposing that the $\psi_{-q / 2}^{(j)}$ and $\Lambda_{m-k / 2-q / 2}^{(r j)}, 0 \leq q<\ell$, have already been determined, we write

$$
\psi_{-\ell / 2}^{\prime(j)}(y, \eta ; .)=\psi_{-\ell / 2,1}^{\prime(j)}(y, \eta ; .)+\psi_{-\ell / 2,2}^{\prime(j)}(y, \eta ; .) \in V_{1}(y, \eta) \oplus V_{1}(y, \eta)^{\perp},
$$


for $1 \leq j \leq d$. The components $\psi_{-\ell / 2,1}^{\prime(j)}$ are uniquely determined by the relations

$$
\begin{aligned}
& \left(\psi_{-\ell / 2,1}^{\prime(j)}(y, \eta ; .), \phi_{0}^{\prime(r)}(y, \eta ; .)\right)_{L^{2}\left(\mathbb{R}^{v}\right)} \\
& \quad=-\sum_{\substack{2|\alpha|+p+q=\ell \\
0 \leq q<\ell}} \frac{1}{\alpha ! i^{|\alpha|}}\left(\partial_{y}^{\alpha} \psi_{-q / 2}^{\prime(j)}(y, \eta ; .), \partial_{\eta}^{\alpha} \phi_{-p / 2}^{\prime(r)}(y, \eta ; .)\right)_{L^{2}\left(\mathbb{R}^{v}\right)} .
\end{aligned}
$$

As for $\Lambda_{m-k / 2-\ell / 2}(y, \eta), 1 \leq r, j \leq d$, we have

$$
\begin{aligned}
& \Lambda_{m-k / 2-\ell / 2}^{(r j)}(y, \eta)=\left(A_{(y, \eta)}^{(k)} \psi_{-\ell / 2,1}^{\prime(j)}(y, \eta ; .), \phi_{0}^{(r)}(y, \eta ; .)\right)_{L^{2}\left(\mathbb{R}^{v}\right)} \\
& \quad-\sum_{\substack { s=1 \\
\begin{subarray}{c}{2|\alpha|+p+q=\ell \\
0 \leq q<\ell{ s = 1 \\
\begin{subarray} { c } { 2 | \alpha | + p + q = \ell \\
0 \leq q < \ell } }\end{subarray}} \frac{1}{\alpha ! i^{|\alpha|}} \partial_{y}^{\alpha} \Lambda_{m-k / 2-q / 2}^{(s j)}(y, \eta)\left(\partial_{\eta}^{\alpha} \phi_{-p / 2}^{(s)}(y, \eta ; .), \phi_{0}^{(r)}(y, \eta ; .)\right)_{L^{2}\left(\mathbb{R}^{v}\right)} \\
& \quad+\sum_{\substack{2|\alpha|+p+q=\ell \\
0 \leq q<\ell}} \frac{1}{\alpha ! i^{|\alpha|}}\left(\partial_{\eta}^{\alpha} A_{(y, \eta)}^{(k+p)} \partial_{y}^{\alpha} \psi_{-q / 2}^{\prime(j)}(y, \eta ; .), \phi_{0}^{(r)}(y, \eta ; .)\right)_{L^{2}\left(\mathbb{R}^{v}\right) .}
\end{aligned}
$$

As for $\psi_{-\ell / 2,2}^{\prime(j)}, 1 \leq j \leq d$, we have

$$
\begin{aligned}
& \psi_{-\ell / 2,2}^{\prime(j)}(y, \eta ; x) \\
& =E_{(y, \eta)}^{(-k)}\left[-A_{(y, \eta)}^{(k)} \psi_{-\ell / 2,1}^{\prime(j)}(y, \eta ; .)+\sum_{r=1}^{d} \phi_{0}^{(r)}(y, \eta ; .) \Lambda_{m-k / 2-\ell / 2}^{(r j)}(y, \eta)\right. \\
& \quad+\sum_{r=1}^{d} \sum_{\substack{|\alpha|+p+q=\ell \\
0 \leq q<\ell}} \frac{1}{\alpha ! i^{|\alpha|}} \partial_{\eta}^{\alpha} \phi_{-p / 2}^{(r)}(y, \eta ; .) \partial_{y}^{\alpha} \Lambda_{m-k / 2-q / 2}^{(r j)}(y, \eta) \\
& \left.\quad-\sum_{2|\alpha|+p+q=\ell} \frac{1}{\alpha ! i|\alpha|} \partial_{\eta}^{\alpha} A_{(y, \eta)}^{(k+p)} \partial_{y}^{\alpha} \psi_{-q / 2}^{\prime(j)}(y, \eta ; .)\right]
\end{aligned}
$$

Although (82), (83) and (84) are extremely complicated, there are instances in which they may be somewhat simplified, as it will be seen later in Section 6.

REMARK 4.4. If

$$
\tilde{H}^{-}=H^{-} R, \quad \tilde{H}^{+}=S H^{+},
$$

where $R, S \in \mathrm{OPS}_{\mathrm{cl}}^{0}\left(\mathbb{R}^{n-v} ; d \times d\right)$ with unitary principal symbols, then the parametrix

$$
\left[\begin{array}{cc}
\tilde{E} & \tilde{K}^{-} \\
\tilde{K}^{+} & -\tilde{\Lambda}
\end{array}\right] \text { of }\left[\begin{array}{cc}
A & \tilde{H}^{-} \\
\tilde{H}^{+} & 0
\end{array}\right]
$$


is given by

$$
\left[\begin{array}{cc}
\text { id } & 0 \\
0 & R^{-1}
\end{array}\right]\left[\begin{array}{cc}
E & K^{-} \\
K^{+} & -\Lambda
\end{array}\right]\left[\begin{array}{cc}
\text { id } & 0 \\
0 & S^{-1}
\end{array}\right]
$$

where $R^{-1}$, respectively $S^{-1}$, is a two-sided parametrix of $R$, respectively $S$. Hence, in particular, we have $\tilde{\Lambda}=R^{-1} \Lambda S^{-1}$. Note, however, that in general for two systems of the form (45),

$$
\left[\begin{array}{cc}
A & H^{-} \\
H^{+} & 0
\end{array}\right], \quad\left[\begin{array}{cc}
A & \tilde{H}^{-} \\
\tilde{H}^{+} & 0
\end{array}\right]
$$

we do not have a ( $\psi \mathrm{do})$ conjugation relation as in (85).

We end this section by explaining how the above arguments can be microlocalized to a conic admissible neighborhood $U \subset T^{*} \mathbb{R}^{n-v} \backslash 0$ of a point $\left(y_{0}, \eta_{0}\right) \in \Sigma$ where $\operatorname{Ker}\left(A_{\left(y_{0}, \eta_{0}\right)}^{(k)}\right) \neq\{0\}$. It is convenient to give the following definition.

DEFINITION 4.5. Given our operator A satisfying $(H 1)$, let $\rho_{0}=\left(y_{0}, \eta_{0}\right) \in \Sigma$ be a point where $\operatorname{dim} \operatorname{Ker}\left(A_{\rho_{0}}^{(k)}\right)=d \geq 1$, and let $U \subset \Sigma$ be an admissible conic neighborhood of $\rho_{0}$. We say that a system

$$
\mathcal{A}=\left[\begin{array}{cc}
A & H^{-} \\
H^{+} & 0
\end{array}\right]
$$

is $a$ system associated with $A$ in $U$ if:

- $H^{-}=\left[H_{1}^{-} \ldots H_{d}^{-}\right], H_{j}^{-} \in \mathrm{OPH}_{\mathrm{cl}}^{0}$ with $\sigma\left(H_{j}^{-}\right) \sim \sum_{\ell \geq 0} \phi_{-\ell / 2}^{(j)}(y, \eta ; x)$, $1 \leq j \leq d$.

- $H^{+}=\left[\begin{array}{c}H_{1}^{+} \\ \vdots \\ H_{d}^{+}\end{array}\right], H_{j}^{+} \in \mathrm{OPH}_{\mathrm{cl}}^{* 0}$ with $\sigma\left(H_{j}^{-}\right) \sim \sum_{\ell \geq 0}{\phi^{\prime}}_{-\ell / 2}^{(j)}(y, \eta ; x), 1 \leq j \leq d$.

- The globally defined $\phi:=\left(\phi_{0}^{(1)}, \ldots, \phi_{0}^{(d)}\right)$, respectively $\phi^{\prime}:=\left(\phi_{0}^{\prime(1)}, \ldots\right.$, \left.${\phi^{\prime}}_{0}^{(d)}\right)$, when $(y, \eta) \in U$ is a gauge of $V_{2} \longrightarrow U$, respectively $V_{1} \longrightarrow U$.

We may now prove the following crucial result.

THEOREM 4.6. Given any system

$$
\mathcal{A}=\left[\begin{array}{cc}
A & H^{-} \\
H^{+} & 0
\end{array}\right]
$$


associated with $A$ in $U$, there exists a system

$$
\mathcal{E}=\left[\begin{array}{cc}
E & K^{-} \\
K^{+} & -\Lambda
\end{array}\right]
$$

where $E \in \mathrm{OPS}_{\mathrm{cl}}^{-m,-k}, \Lambda=\Lambda\left(y, D_{y}\right)=\left(\Lambda^{(r j)}\left(y, D_{y}\right)\right)_{r, j=1, \ldots, d}$ is $a$ $d \times d$ pseudodifferential system whose entries $\Lambda^{(r j)} \in \operatorname{OPS}_{\mathrm{cl}}^{m-k / 2}\left(\mathbb{R}^{n-v}\right), K^{-}=$ $\left[K_{1}^{-} \ldots K_{d}^{-}\right], K_{j}^{-} \in \mathrm{OPH}_{\mathrm{cl}}^{0}, K^{+}=\left[\begin{array}{c}K_{1}^{+} \\ \vdots \\ K_{d}^{+}\end{array}\right], K_{j}^{+} \in \mathrm{OPH}_{\mathrm{cl}}^{* 0}, 1 \leq j \leq d$, which is a two-sided microlocal parametrix for $\mathcal{A}$ at $\rho_{0}$, i.e. there is a conic neighborhood $\Gamma \subset \subset U$ of $\left(y_{0}, \eta_{0}\right)$ such that for any given $f \in \mathcal{E}^{\prime}\left(\mathbb{R}^{n}\right)$ with $\mathrm{WF}(f) \subset \tilde{\Gamma}:=$ $\{(x, y, \xi, \eta) ;(y, \eta) \in \Gamma\}$ and any given

$$
g=\left[\begin{array}{c}
g_{1} \\
\vdots \\
g_{d}
\end{array}\right] \in \mathcal{E}^{\prime}\left(\mathbb{R}^{n-v} ; \mathbb{C}^{d}\right)
$$

with $\mathrm{WF}(g):=\bigcup_{j=1}^{d} \mathrm{WF}\left(g_{j}\right) \subset \Gamma$, we have

$$
\mathcal{E} \mathcal{A}\left[\begin{array}{l}
f \\
g
\end{array}\right]-\left[\begin{array}{l}
f \\
g
\end{array}\right], \mathcal{A \mathcal { E }}\left[\begin{array}{l}
f \\
g
\end{array}\right]-\left[\begin{array}{l}
f \\
g
\end{array}\right] \begin{gathered}
C^{\infty}\left(\mathbb{R}^{n}\right) \\
C^{\infty}\left(\mathbb{R}^{n-v} ; \mathbb{C}^{d}\right)
\end{gathered} .
$$

Proof. We start by taking

$$
\mathcal{E}=\left[\begin{array}{cc}
E & K^{-} \\
K^{+} & -\Lambda
\end{array}\right]
$$

as in the statement with symbols $\sigma\left(\Lambda^{(r j)}\right) \sim \sum_{\ell \geq 0} \Lambda_{m-k / 2-\ell / 2}^{(r j)}(y, \eta), \sigma\left(K_{j}^{-}\right) \sim$ $\sum_{\ell \geq 0} \psi_{-\ell / 2}^{\prime(j)}(y, \eta ; x)$, and $\sigma\left(K_{j}^{+}\right) \sim \sum_{\ell \geq 0} \psi_{-\ell / 2}^{(j)}(y, \eta ; x), 1 \leq j, r \leq d$, where $\psi_{0}^{\prime(j)}=\phi_{0}^{\prime(j)}$ and $\psi_{0}^{(j)}=\phi_{0}^{(j)}, 1 \leq j \leq d$. Write

$$
\mathcal{A E}=\left[\begin{array}{cc}
A E+H^{-} K^{+} & A K^{-}-H^{-} \Lambda \\
H^{+} E & H^{+} K^{-}
\end{array}\right]
$$

and note that by the calculus in Section 3 the full symbols of the entries of $\mathcal{A E}$ are given by the left-hand sides of $\mathbf{1}^{\prime}$ through $\mathbf{4}^{\prime}$ (in the proof of Theorem 4.1). 
Fix now a conic neighborhood $\Gamma \subset \subset U$ of $\left(y_{0}, \eta_{0}\right)$. The proof of Theorem 4.1 shows that we may globally construct the operators $E_{(y, \eta)}^{(-k+q)}$ and the symbols $\psi_{-q / 2}^{(j)}(y, \eta ; x), \psi_{-q / 2}^{\prime(j)}(y, \eta ; x), \Lambda_{m-k / 2-q / 2}^{(r j)}(y, \eta), q \geq 0$ and $1 \leq j, r \leq d$, in such a way that equations $\mathbf{1}^{\prime}-\mathbf{4}^{\prime}$ are satisfied in $\tilde{\Gamma}=\{(x, y, \xi, \eta) ;(y, \eta) \in \Gamma\}$. As a consequence, taking into account Lemma 3.16, given any $f$ and $g$ as in the statement, we have

$$
\begin{gathered}
H^{+} E f \in C^{\infty}\left(\mathbb{R}^{n} ; \mathbb{C}^{d}\right), \\
\left(A K^{-}-H^{-} \Lambda\right) g \in C^{\infty}\left(\mathbb{R}^{n}\right), \\
\left(H^{+} K^{-}-\mathrm{id}\right) g \in C^{\infty}\left(\mathbb{R}^{n-\nu} ; \mathbb{C}^{d}\right), \\
\left(A E+\sum_{j=1}^{d} H_{j}^{-} K_{j}^{+}-\mathrm{id}\right) f-R f \in C^{\infty}\left(\mathbb{R}^{n}\right),
\end{gathered}
$$

where the symbol of the $\psi$ do $R$ belongs to $S^{0, \infty}(\tilde{\Gamma})$. As in the first step of the proof of Theorem 4.1, we can reabsorb $R$ into $E$ in such a way that

$$
\left(A E+\sum_{j=1}^{d} H_{j}^{-} K_{j}^{+}-\mathrm{id}\right) f \in C^{\infty}\left(\mathbb{R}^{n}\right) .
$$

By a similar argument, we can construct a system $\mathcal{E}^{\prime}$ which is a microlocal leftparametrix for $\mathcal{A}$ at $\rho_{0}$. As usual, $\mathcal{E}$ and $\mathcal{E}^{\prime}$ coincide microlocally.

REMARK 4.7. Observe that the full symbol of $\Lambda$ in $\Gamma$ is given by relations (81) and (83).

\section{Hypoellipticity results and other applications}

As an immediate consequence of Theorem 4.6, we have the following hypoellipticity result.

THEOREM 5.1. Given our operator A satisfying $(H 1)$, let $\rho_{0}=\left(y_{0}, \eta_{0}\right) \in \Sigma$ be a point where $\operatorname{Ker}\left(A_{\rho_{0}}^{(k)}\right) \neq\{0\}$, let

$$
\mathcal{A}=\left[\begin{array}{cc}
A & H^{-} \\
H^{+} & 0
\end{array}\right]
$$


be any system associated with $A$ in $U$ and let the system

$$
\mathcal{E}=\left[\begin{array}{cc}
E & K^{-} \\
K^{+} & -\Lambda
\end{array}\right]
$$

be a two-sided (microlocal) parametrix of $\mathcal{A}$ at $\rho_{0}$.

Then $A$ is hypoelliptic at $\rho_{0}$ with loss of $\frac{k}{2}+r$ derivatives $(r>0)$, respectively hypoelliptic at $\rho_{0}$, if and only if $\Lambda$ is hypoelliptic at $\rho_{0}$ with loss of $r$ derivatives, respectively hypoelliptic at $\rho_{0}$.

Proof. Sufficiency. By hypothesis, there is a conic neighborhood $\Gamma \subset \subset U$ of $\rho_{0}$ such that

$$
\mathcal{E} \mathcal{A}\left[\begin{array}{l}
f \\
g
\end{array}\right]-\left[\begin{array}{l}
f \\
g
\end{array}\right] \in \begin{gathered}
C^{\infty}\left(\mathbb{R}^{n}\right) \\
C^{\infty}\left(\mathbb{R}^{n-v} ; \mathbb{C}^{d}\right)
\end{gathered},
$$

whenever $f \in \mathcal{E}^{\prime}\left(\mathbb{R}^{n}\right), g \in \mathcal{E}^{\prime}\left(\mathbb{R}^{n-v} ; \mathbb{C}^{d}\right)$ are such that $\mathrm{WF}(f) \subset \tilde{\Gamma}, \mathrm{WF}(g) \subset \Gamma$. Suppose now that $A f \in H^{t}$ at $\rho_{0}$ (see Remark 1.2). From Lemmas 3.16 and 3.17, we have $E A f \in H^{t+m-k / 2}$ at $\rho_{0}$, and since $E A f+K^{-} H^{+} f-f \in C^{\infty}\left(\mathbb{R}^{n}\right)$, we get $f-K^{-} H^{+} f \in H^{t+m-k / 2}$ at $\rho_{0}$. On the other hand $K^{+} A f \in H^{t}$ at $\rho_{0}$ and since $K^{+} A f-\Lambda H^{+} f \in C^{\infty}\left(\mathbb{R}^{n-v} ; \mathbb{C}^{d}\right)$, we obtain $\Lambda H^{+} f \in H^{t}$ at $\rho_{0}$. Thus, $H^{+} f \in H^{t+m-k / 2-r}$ at $\rho_{0}$. By continuity, $K^{-} H^{+} f \in H^{t+m-k / 2-r}$ at $\rho_{0}$. Hence, $f \in H^{t+m-k / 2-r}$ at $\rho_{0}$.

Necessity. By hypothesis we have also

$$
\mathcal{A E}\left[\begin{array}{l}
f \\
g
\end{array}\right]-\left[\begin{array}{l}
f \\
g
\end{array}\right] \in \begin{gathered}
C^{\infty}\left(\mathbb{R}^{n}\right) \\
\times \\
C^{\infty}\left(\mathbb{R}^{n-v} ; \mathbb{C}^{d}\right)
\end{gathered},
$$

whenever $f$ and $g$ are as above. Suppose now that $\Lambda g \in H^{t}$ at $\rho_{0}$. Then (again by Lemmas 3.16 and 3.17) we have $H^{-} \Lambda g \in H^{t}$ at $\rho_{0}$ and since $A K^{-} g-H^{-} \Lambda g \in$ $C^{\infty}\left(\mathbb{R}^{n}\right)$, we get $A K^{-} g \in H^{t}$ at $\rho_{0}$. Thus, $K^{-} g \in H^{t+m-k / 2-r}$ at $\rho_{0}$. As $H^{+} K^{-} g-g \in C^{\infty}\left(\mathbb{R}^{n-v} ; \mathbb{C}^{d}\right)$, we conclude that $g \in H^{t+m-k / 2-r}$ at $\rho_{0}$.

The same arguments show that $A$ is hypoelliptic at $\rho_{0}$ if and only if $\Lambda$ is hypoelliptic at $\rho_{0}$.

In the next theorem, we collect some interesting results that are trivial consequences of the proof of Theorem 5.1.

THEOREM 5.2. Let $\mathcal{A}$ be any fixed system associated with $A$ in $U$ and let $\mathcal{E}$ be a two-sided microlocal parametrix for $\mathcal{A}$ as in Theorem 4.6. 
(a) (Existence of a micro-parametrix for A.) Suppose that, for some $r>$ 0 , there exists a $d \times d$ system of $\psi \operatorname{dos} G=\left(G_{j l}\right)_{1 \leq j, l \leq d}$ with $G_{j l} \in$ $\operatorname{OPS}_{1 / 2,1 / 2}^{-(m-k / 2)+r}\left(\mathbb{R}^{n-v}\right), 1 \leq j, l \leq d$, such that, for all $g \in \mathcal{E}^{\prime}\left(\mathbb{R}^{n-v} ; \mathbb{C}^{d}\right)$ with $\mathrm{WF}(g) \subset \Gamma$, we have

$$
\Lambda G g-g \in C^{\infty}\left(\mathbb{R}^{n-v} ; \mathbb{C}^{d}\right)
$$

(respectively $G \Lambda g-g \in C^{\infty}\left(\mathbb{R}^{n-v} ; \mathbb{C}^{d}\right)$ ). Then, for all $f \in \mathcal{E}^{\prime}\left(\mathbb{R}^{n}\right)$ with $\mathrm{WF}(f) \subset \tilde{\Gamma}$, we have

$$
A\left(E+K^{-} G K^{+}\right) f-f \in C^{\infty}\left(\mathbb{R}^{n}\right)
$$

(respectively $\left(E+K^{-} G K^{+}\right) A f-f \in C^{\infty}\left(\mathbb{R}^{n}\right)$ ).

(b) (Propagation of singularities.) Suppose there exists a set $T \subset \Gamma \cap \operatorname{Char}(\Lambda)=$ $\left\{(y, \eta) \in \Gamma ; \operatorname{det} \Lambda_{m-k / 2}(y, \eta)=0\right\}$, such that $\left(y_{0}, \eta_{0}\right) \in T \cap\left(\overline{T \backslash\left\{\left(y_{0}, \eta_{0}\right)\right\}}\right)$ and suppose that $\Lambda$ satisfies the following property: For all $g \in \mathcal{E}^{\prime}\left(\mathbb{R}^{n-v} ; \mathbb{C}^{d}\right)$,

$$
\mathrm{WF}(\Lambda g) \cap \Gamma=\emptyset,\left(y_{0}, \eta_{0}\right) \in \mathrm{WF}(g) \Longrightarrow T \subset \mathrm{WF}(g) .
$$

Then, upon denoting by

$$
\Gamma_{0}:=\{(0, y, 0, \eta) ;(y, \eta) \in \Gamma\}, \quad T_{0}:=\{(0, y, 0, \eta) ;(y, \eta) \in T\},
$$

we have: For all $f \in \mathcal{E}^{\prime}\left(\mathbb{R}^{n}\right)$,

$$
\mathrm{WF}(A f) \cap \Gamma_{0}=\emptyset, \quad\left(0, y_{0}, 0, \eta_{0}\right) \in \mathrm{WF}(f) \Longrightarrow T_{0} \subset \mathrm{WF}(f) .
$$

Proof. (a) Suppose $\Lambda G g-g \in C^{\infty}\left(\mathbb{R}^{n-v} ; \mathbb{C}^{d}\right)$ whenever $\operatorname{WF}(g) \subset \Gamma$. Since

$$
\mathcal{E}=\left[\begin{array}{cc}
E & K^{-} \\
K^{+} & -\Lambda
\end{array}\right]
$$

is a right-parametrix for $\mathcal{A}$, we have $A E f=f-H^{-} K^{+} f+C^{\infty}\left(\mathbb{R}^{n}\right)$ whenever $\mathrm{WF}(f) \subset \tilde{\Gamma}$. On the other hand,

$$
A K^{-} G K^{+} f=H^{-} \Lambda G K^{+} f+C^{\infty}\left(\mathbb{R}^{n}\right)=H^{-} K^{+} f+C^{\infty}\left(\mathbb{R}^{n}\right) .
$$

Hence, $E+K^{-} G K^{+}$is a right-parametrix for $A$. In the same way, one proves the other case.

(b) We use the fact that $\mathcal{E}$ is a left-parametrix for $\mathcal{A}$. Take any $f \in \mathcal{E}^{\prime}\left(\mathbb{R}^{n}\right)$ with $\mathrm{WF}(A f) \cap \Gamma_{0}=\varnothing$ and $\left(0, y_{0}, 0, \eta_{0}\right) \in \mathrm{WF}(f)$. Since $\mathrm{WF}(E A f) \subset \mathrm{WF}(A f)$, we have $\operatorname{WF}(E A f) \cap \Gamma_{0}=\emptyset$. As $E A f=f-K^{-} H^{+} f+C^{\infty}\left(\mathbb{R}^{n}\right)$, we obtain

$$
\mathrm{WF}(f) \cap \Gamma_{0}=\operatorname{WF}\left(K^{-} H^{+} f\right) \cap \Gamma_{0} .
$$


The $\mathrm{WF}^{\prime}$ relation for $K^{-}$yields

$$
\mathrm{WF}\left(K^{-} H^{+} f\right) \cap \Gamma_{0} \subset\left\{(0, y, 0, \eta) ;(y, \eta) \in \Gamma \cap \mathrm{WF}\left(H^{+} f\right)\right\} .
$$

On the other hand, the $\mathrm{WF}^{\prime}$ relation for $H^{+}$gives

$$
\left\{(0, y, 0, \eta) ;(y, \eta) \in \Gamma \cap \mathrm{WF}\left(H^{+} f\right)\right\} \subset \mathrm{WF}(f) \cap \Gamma_{0} .
$$

Hence,

$$
(0, y, 0, \eta) \in \mathrm{WF}(f) \cap \Gamma_{0} \Longleftrightarrow(y, \eta) \in \mathrm{WF}\left(H^{+} f\right) \cap \Gamma .
$$

By hypothesis, $\operatorname{WF}\left(K^{+} A f\right) \cap \Gamma=\emptyset$ and since $K^{+} A f=\Lambda H^{+} f+C^{\infty}\left(\mathbb{R}^{n-v} ; \mathbb{C}^{d}\right)$, we get $\operatorname{WF}\left(\Lambda H^{+} f\right) \cap \Gamma=\emptyset$. On the other hand, by hypothesis, $\left(0, y_{0}, 0, \eta_{0}\right) \in$ $\mathrm{WF}(f)$, whence $(86)$ yields $\left(y_{0}, \eta_{0}\right) \in \mathrm{WF}\left(H^{+} f\right)$. By the propagation property of $\Lambda$, we conclude that $T \subset \mathrm{WF}\left(H^{+} f\right)$ whence, again by (86), $T_{0} \subset \mathrm{WF}(f)$.

It is worth noting that not only is the hypoellipticity/propagation of singularities of $A$ related to that of $\Lambda$ but also lower bounds of $A$ are related to lower bounds of $\Lambda$. We make this precise in a simplified setup (which, however, gives the idea of more general results).

Suppose that $A_{\rho}^{(k)}=\left(A_{\rho}^{(k)}\right)^{*} \geq 0$ for all $\rho \in \Sigma$ (so that (H1) holds), and suppose we may find an admissible (conic) set $U$ of the form $U=\Omega \times\left(\mathbb{R}^{n-v} \backslash\{0\}\right)$, $y_{0} \in \Omega \subset \mathbb{R}^{n-v}$ being open. Note that, in this case, $V_{1}(\rho)=V_{2}(\rho)$ for all $\rho \in U$ and that the $d \times d$ matrix $\Lambda_{m-k / 2}(y, \eta)$ is Hermitian and nonnegative. Consider now a system

$$
\mathcal{A}=\left[\begin{array}{cc}
A & H^{-} \\
H^{+} & 0
\end{array}\right]
$$

associated with $A$ on $U$, where $H^{+}=\left(H^{-}\right)^{*}$ and the terms of order $-j / 2, j \geq 1$, in the symbol of $\mathrm{H}^{-}$are chosen to be zero. Theorem 4.6 ensures the existence of a system

$$
\mathcal{E}=\left[\begin{array}{cc}
E & K^{-} \\
K^{+} & -\Lambda
\end{array}\right]
$$

such that for some neighborhood $\omega$ of $y_{0}, \omega \subset \subset \Omega$, we have

$$
\mathcal{A E}\left[\begin{array}{l}
f \\
g
\end{array}\right]-\left[\begin{array}{l}
f \\
g
\end{array}\right], \mathcal{E} \mathcal{A}\left[\begin{array}{l}
f \\
g
\end{array}\right]-\left[\begin{array}{l}
f \\
g
\end{array}\right] \underset{C^{\infty}\left(\mathbb{R}^{n-v} ; \mathbb{C}^{d}\right)}{C^{\infty}\left(\mathbb{R}^{n}\right)},
$$


for all $f \in \mathcal{E}^{\prime}\left(\mathbb{R}^{n}\right)$ and $g \in \mathcal{E}^{\prime}\left(\mathbb{R}^{n-v} ; \mathbb{C}^{d}\right)$ with

$\mathrm{WF}(g) \subset \omega \times\left(\mathbb{R}^{n-v} \backslash\{0\}\right)=: \Gamma, \operatorname{WF}(f) \subset\{(x, y, \xi, \eta) ; y \in \omega, \eta \neq 0\}=: \tilde{\Gamma}$.

One then has the following result.

THEOREM 5.3. Suppose that, for any given $\omega^{\prime} \subset \subset \omega$ and any $\mu<(m-k / 2-1) / 2$, there are constants $c>0, C \geq 0$ such that

$$
\operatorname{Re}(\Lambda g, g) \geq c\|g\|_{(m-k / 2-1) / 2}^{2}-C\|g\|_{\mu}^{2}, \quad \text { for all } g \in C_{0}^{\infty}\left(\omega^{\prime} ; \mathbb{C}^{d}\right) .
$$

Then, for any $\omega^{\prime}$ and $\mu$ as above, there are constants $c^{\prime}>0, C^{\prime} \geq 0$ such that

$$
\operatorname{Re}(A f, f) \geq c^{\prime}\|f\|_{(m-k / 2-1) / 2}^{2}-C^{\prime}\|f\|_{\mu}^{2}, \quad \text { for all } f \in C_{0}^{\infty}\left(\mathbb{R}^{v} \times \omega^{\prime}\right) .
$$

Proof. Write $f=K^{-} H^{+} f+\left(\mathrm{id}-K^{-} H^{+}\right) f$. Then

$$
\begin{aligned}
\operatorname{Re}(A f, f)= & \operatorname{Re}\left(A K^{-} H^{+} f, K^{-} H^{+} f\right)+2 \operatorname{Re}\left(A K^{-} H^{+} f, f-K^{-} H^{+} f\right) \\
& +\operatorname{Re}\left(A\left(f-K^{-} H^{+} f\right), f-K^{-} H^{+} f\right)=(1)+(2)+(3) .
\end{aligned}
$$

Estimating (1). We have $A K^{-} H^{+} f=H^{-} \Lambda H^{+} f+\operatorname{smoothing}(f)$, so that

$$
\begin{aligned}
(1) & =\operatorname{Re}\left(H^{-} \Lambda H^{+} f+\operatorname{smoothing}(f), K^{-} H^{+} f\right) \\
& =\operatorname{Re}\left(\Lambda H^{+} f, H^{+} K^{-} H^{+} f\right)+O\left(\|f\|_{\mu}^{2}\right) .
\end{aligned}
$$

Since $H^{+} K^{-}-$id is smoothing on $\omega$, we get

$$
(1)=\operatorname{Re}\left(\Lambda H^{+} f, H^{+} f\right)+O\left(\|f\|_{\mu}^{2}\right) \geq c\left\|H^{+} f\right\|_{(m-k / 2-1) / 2}^{2}-C^{\prime}\|f\|_{\mu}^{2}
$$

for some $C^{\prime} \geq 0$ (we will use the same $C^{\prime}$ to denote a suitable nonnegative constant, possibly different at each occurrence). By the continuity of $K^{-}$, we finally have

$$
\text { (1) }=\operatorname{Re}\left(A K^{-} H^{+} f, K^{-} H^{+} f\right) \geq c_{1}\left\|K^{-} H^{+} f\right\|_{(m-k / 2-1) / 2}^{2}-C^{\prime}\|f\|_{\mu}^{2},
$$
where $c_{1}>0$.

Estimating (2). We have

$$
\begin{aligned}
\left(A K^{-}\right. & \left.H^{+} f, f-K^{-} H^{+} f\right) \\
& =\left(H^{-} \Lambda H^{+} f+\operatorname{smoothing}(f), E A f+\operatorname{smoothing}(f)\right) \\
& =\left(\Lambda H^{+} f, H^{+} E A f\right)+O\left(\|f\|_{\mu}^{2}\right) .
\end{aligned}
$$

Since $H^{+} E$ is smoothing on $\mathbb{R}^{v} \times \omega$, we conclude that

$$
\text { (2) }=2 \operatorname{Re}\left(A K^{-} H^{+} f, f-K^{-} H^{+} f\right)=O\left(\|f\|_{\mu}^{2}\right) .
$$


Estimating (3). Since $K^{-} H^{+}\left(\mathrm{id}-K^{-} H^{+}\right)$is smoothing on $\mathbb{R}^{v} \times \omega$, we may write

$$
\text { (3) }=\operatorname{Re}\left(\left(A+K^{-} H^{+}\right)\left(f-K^{-} H^{+} f\right), f-K^{-} H^{+} f\right)+O\left(\|f\|_{\mu}^{2}\right) .
$$

By Proposition 7.1 of [16], we have

$$
\begin{gathered}
\operatorname{Re}\left(\left(A+K^{-} H^{+}\right)\left(f-K^{-} H^{+} f\right), f-K^{-} H^{+} f\right) \\
\geq c_{2}\left\|f-K^{-} H^{+} f\right\|_{(m-k / 2-1) / 2}^{2}-C^{\prime}\|f\|_{\mu}^{2},
\end{gathered}
$$

where $c_{2}>0$. Since

$\|f\|_{(m-k / 2-1) / 2}^{2}=\left\|K^{-} H^{+} f\right\|_{(m-k / 2-1) / 2}^{2}+\left\|f-K^{-} H^{+} f\right\|_{(m-k / 2-1) / 2}^{2}+O\left(\|f\|_{\mu}^{2}\right)$, we obtain (88).

REMARK 5.4. Inequality (87) is usually called strong Melin's inequality. We may call inequality (88) a strong Hörmander inequality, which when $k=2$ is a sharpened form of the classical Hörmander inequality (see [10, Vol. III, Theorem 22.3.2]), and for $k \geq 4$ is the generalization dealt with in [16]. Actually, in [16] (see also [17]) we have given, in a more general setup, sufficient conditions in order to have the weaker inequality

$$
\operatorname{Re}(A f, f) \geq-c^{\prime}\|f\|_{(m-k / 2-1) / 2}^{2}, \quad c^{\prime}>0 .
$$

In the present setting, inequality (88) is indeed much stronger, so that it is natural to ask when (87) holds. We do not know the answer in general but there is at least one classical and meaningful case which we can handle (see [2] and [4]). Suppose that the principal symbol of $H^{ \pm}$on $U$ (admissible) is a gauge $\phi=\left(\phi_{0}^{(1)}, \ldots, \phi_{0}^{(d)}\right)$ of $V_{1}=V_{2} \longrightarrow U$, such that the $\phi_{0}^{(j)}(y, \eta ;),. 1 \leq j \leq d$, are either all even or all odd in the variable $x$. Suppose finally that $\left.A_{(y, \eta)}^{(k)}\right|_{V_{1}(y, \eta)}=\lambda(y, \eta) \operatorname{id}_{V_{1}(y, \eta)}$. In this case, by virtue of the choice of $H^{-}$and Remark 4.3, we have $\Lambda_{m-k / 2-1 / 2}(y, \eta)=0$ on $U$, so that the symbol of $\Lambda$ has an expansion on $\Gamma$ of the form

$$
\sigma(\Lambda)(y, \eta)=\Lambda_{m-k / 2}(y, \eta)+\Lambda_{m-k / 2-1}(y, \eta)+\cdots,
$$

where

$$
\Lambda_{m-k / 2}(y, \eta)=\lambda(y, \eta) I_{\mathbb{C}^{d}}, \quad \text { for all }(y, \eta) \in U
$$

Hence,

$$
\operatorname{Char}(\Lambda) \cap \Gamma=\{(y, \eta) \in \Gamma ; \lambda(y, \eta)=0\}=: \Sigma_{\lambda} .
$$

Suppose, furthermore, that the set $\Sigma_{\lambda}$ is a smooth closed conic submanifold of $T^{*} \mathbb{R}^{n-v} \backslash 0$ (on which the canonical 1-form $\sum_{j=1}^{n-v} \eta_{j} d y_{j}$ does not vanish identically), 
and that $\lambda$ vanishes exactly to order two on $\Sigma_{\lambda}$. Under all these assumptions, in [14] and [16] (see also [2] and [4]), it is shown how to associate with $\Lambda$, at every $\rho \in \Sigma_{\lambda}$, a localized operator $\Lambda_{\rho}^{(2)}$ which depends only on $\Lambda_{m-k / 2}$ and $\Lambda_{m-k / 2-1}$. In [14] and [16] it is proved that inequality (87) holds if and only if

$$
\operatorname{Re}\left(\Lambda_{\rho}^{(2)}\right)=\frac{\Lambda_{\rho}^{(2)}+\left(\Lambda_{\rho}^{(2)}\right)^{*}}{2}>0, \quad \text { for all } \rho \in \Sigma_{\lambda} .
$$

Hence, the content of Theorem 5.3 is that if we have stronger information on the way $\lambda$ vanishes and the highly non-trivial information that $\operatorname{Re}\left(\Lambda_{\rho}^{(2)}\right)>0$, we obtain the stronger inequality (88).

REMARK 5.5. All the preceding constructions were carried out on supposing that $A$ is transversally elliptic, i.e. condition (2) holds. On the other hand, it can immediately be seen that the validity of (2) is equivalent to the validity for all $\rho \in \Sigma$ of

$$
(x, \xi) \in T^{*} \mathbb{R}^{v} \backslash\{(0,0)\} \Longrightarrow \tilde{a}_{\rho}(x, \xi) \neq 0
$$

$\left(\tilde{a}_{\rho}\right.$ having been defined in (6)). Note that if $\left(2^{\prime}\right)_{\rho_{0}}$ holds, then, by homogeneity, $\left(2^{\prime}\right)_{\rho}$ holds for all $\rho$ in a conic neighborhood of $\rho_{0}$ in $\Sigma$.

Moreover, condition $\left(2^{\prime}\right)_{\rho}$ is exactly what ensures that the localized operator $A_{\rho}$ be globally elliptic in the sense of Shubin. Now Theorem 1.4 can be restated in the following form.

THEOREM $1.4^{\prime}$. Let A satisfy (1) and suppose that $\left(2^{\prime}\right)_{\rho_{0}}$ holds for some $\rho_{0} \in \Sigma$. Then $A$ is hypoelliptic at $\rho_{0}$ with loss of $k / 2$ derivatives if and only if $\operatorname{Ker}\left(A_{\rho_{0}}\right)=\{0\}$.

Hence, we can take A to satisfy (1) and $\left(2^{\prime}\right)_{\rho_{0}}$, and suppose (H1) to be fulfilled in a neighborhood $U \subset \Sigma$ of $\rho_{0}$ (that we may suppose admissible). Then Theorem 4.6 and Theorems 5.1-5.3 still hold true.

At this point, we can easily remove the restriction of having $A$ defined in the whole $\mathbb{R}_{z}^{n}=\mathbb{R}_{x}^{v} \times \mathbb{R}_{y}^{n-v}$ and suppose that $A$ is defined only on some open set $\Omega \subset \mathbb{R}^{n}$ such that $\Omega \cap\{x=0\} \neq \varnothing$, condition (1) holds on $T^{*} \Omega \backslash 0$ and with $\Sigma=\left\{(z, \zeta) \in T^{*} \Omega \backslash 0 ; x=\xi=0\right\}$. Suppose that, for some $\rho_{0} \in \Sigma$ condition $\left(2^{\prime}\right) \rho_{0}$ holds and that $\operatorname{Ker}\left(A_{\rho_{0}}\right) \neq\{0\}$. We extend $A$ to an operator $\tilde{A}$ defined in the whole $\mathbb{R}^{n}$ in the following way. Define $B\left(z, D_{z}\right)$ as follows (recall that $k$ must be even):

$$
B\left(z, D_{z}\right):=\left|D_{z}\right|^{(m-k) / 2}\left(\sum_{j=1}^{v}\left(D_{x_{j}}^{2}+x_{j}^{2}\left|D_{y}\right|^{2}\right)\right)^{k / 2}\left|D_{z}\right|^{(m-k) / 2} .
$$

Note that $B\left(z, D_{z}\right) \in \operatorname{OPS}_{\mathrm{cl}}^{m}\left(\mathbb{R}^{n}\right)$ vanishes to order $k$ for $x=\xi=0$ and is transversally elliptic. Fix now a cut-off $\chi=\chi(z, \zeta)$, homogeneous of degree zero in 
$\zeta, 0 \leq \chi \leq 1$, such that $\chi$ is supported in a full conic neighborhood of $\rho_{0}$ contained in $T^{*} \Omega \backslash 0$, and $\chi \equiv 1$ in a smaller full conic neighborhood of $\rho_{0}$. Put, for $f \in C_{0}^{\infty}\left(\mathbb{R}^{n}\right)$,

$$
\tilde{A}\left(z, D_{z}\right) f:=A\left(z, D_{z}\right)\left(\chi\left(z, D_{z}\right) f\right)+B\left(z, D_{z}\right)\left[\left(1-\chi\left(z, D_{z}\right)\right) f\right] .
$$

Obviously $\tilde{A} \in \operatorname{OPS}_{\mathrm{cl}}^{m}\left(\mathbb{R}^{n}\right)$, vanishes to order $k$ for $x=\xi=0$ and the hypoellipticity of $A$ at $\rho_{0}$ is equivalent to the hypoellipticity of $\tilde{A}$ at $\rho_{0}$. Hence, on supposing that (H1) holds for $A$ in a neighborhood $\tilde{U} \subset\left\{(y, \eta) \in T^{*} \mathbb{R}^{n-v} ;(0, y) \in \Omega,|\eta|=1\right\}$, all the mentioned results still hold true for $A$.

REMARK 5.6. All the constructions above deal with the $C^{\infty}$-hypoellipticity. Suppose now that $A$ is an analytic $\psi$ do (see, for instance, Treves' book [21]). It is known (Métivier [13]) that when $A_{\rho}$ is injective, then $A$ is microlocally analytic hypoelliptic at $\rho$. The natural question is, therefore, the following:

- Given a system $\left[\begin{array}{cc}A & H^{-} \\ H^{+} & 0\end{array}\right]$ associated with $A$, and its parametrix $\left[\begin{array}{cc}E & K^{-} \\ K^{+} & -\Lambda\end{array}\right]$, is it true that $\Lambda$ is an analytic system of $\psi$ dos? And furthermore, if this is the case, is the analytic hypoellipticity of $A$ at $\rho$ equivalent to the analytic hypoellipticity of $\Lambda$ at $\rho$ ?

We do not know the answer. However, the results of Stein [20] and Grigis-Rothschild [5] (see also Kwon [12]) allow one to conjecture that the answer is indeed positive.

\section{Examples}

In this section, we collect a number of simple, yet meaningful, examples in which the machinery developed earlier is put to work.

In all the following examples, we shall deal with situations in which $\left[A_{\rho}, A_{\rho}^{*}\right]=$ 0 , so that the vector-bundles $V_{1}$ and $V_{2}$ (see Definition 2.3) coincide. Furthermore, we shall choose systems

$$
\mathcal{A}=\left[\begin{array}{cc}
A & H^{-} \\
H^{+} & 0
\end{array}\right]
$$

associated with $A$ (see Definition 4.5) where, for all $j=1, \ldots, d=\operatorname{dim} \operatorname{Ker}\left(A_{\rho_{0}}\right)$, we take $\sigma\left(H_{j}^{ \pm}\right)=\phi_{0}^{(j)}(y, \eta ; x)=\phi_{0}^{(j)}(y, \eta ; x)$, with $\phi=\left(\phi_{0}^{(1)}, \ldots, \phi_{0}^{(d)}\right)$ an orthonormal gauge of $V_{1}=V_{2}$. Hence, if

$$
\mathcal{E}=\left[\begin{array}{cc}
E & K^{-} \\
K^{+} & -\Lambda
\end{array}\right]
$$


is the microlocal parametrix of $\mathcal{A}$ constructed in Theorem 4.6, the principal symbol of $K_{j}^{ \pm}$is $\phi_{0}^{(j)}, 1 \leq j \leq d$.

In the following, with the exception of Example 6.5, we will limit ourselves to considering hypoellipticity with loss of $1+k / 2$ derivatives, so that only the first three terms in the symbol of $\Lambda\left(y, D_{y}\right)$ will matter. For convenience, we thus recall how formula (83) reads in the present setting for $\ell=0,1,2$. We have

$$
\left\{\begin{aligned}
\Lambda_{m-k / 2}^{(r j)}(\rho)= & \left(A_{\rho}^{(k)} \phi_{0}^{(j)}(\rho ; .), \phi_{0}^{(r)}(\rho ; .)\right)_{L^{2}\left(\mathbb{R}^{\nu}\right)} \\
\Lambda_{m-k / 2-1 / 2}^{(r j)}(\rho) & =\left(A_{\rho}^{(k+1)} \phi_{0}^{(j)}(\rho ; .), \phi_{0}^{(r)}(\rho ; .)\right)_{L^{2}\left(\mathbb{R}^{v}\right)} \\
\Lambda_{m-k / 2-1}^{(r j)}(\rho)= & \left(A_{\rho}^{(k)} \psi_{-1,1}^{\prime(j)}(\rho ; .), \phi_{0}^{(r)}(\rho ; .)\right)_{L^{2}\left(\mathbb{R}^{v}\right)} \\
& +i \sum_{s=1}^{d} \sum_{|\alpha|=1} \partial_{y}^{\alpha} \Lambda_{m-k / 2}^{(s j)}(\rho)\left(\partial_{\eta}^{\alpha} \phi_{0}^{(s)}(\rho ; .), \phi_{0}^{(r)}(\rho ; .)\right)_{L^{2}\left(\mathbb{R}^{v}\right)} \\
& +\left(A_{\rho}^{(k+1)} \psi_{-1 / 2}^{(j)}(\rho ; .), \phi_{0}^{(r)}(\rho ; .)\right)_{L^{2}\left(\mathbb{R}^{v}\right)} \\
& +\left(A_{\rho}^{(k+2)} \phi_{0}^{(j)}(\rho ; .), \phi_{0}^{(r)}(\rho ; .)\right)_{L^{2}\left(\mathbb{R}^{v}\right)} \\
& +\frac{1}{i} \sum_{|\alpha|=1}\left(\partial_{\eta}^{\alpha} A_{\rho}^{(k)} \partial_{y}^{\alpha} \phi_{0}^{(j)}(\rho ; .), \phi_{0}^{(r)}(\rho ; .)\right)_{L^{2}\left(\mathbb{R}^{\nu}\right)}
\end{aligned}\right.
$$

where $1 \leq j, r \leq d$ and

$$
\left\{\begin{array}{l}
\psi_{-1,1}^{\prime(j)}(\rho ; .)=-\frac{1}{i} \sum_{r=1}^{d} \sum_{|\alpha|=1}\left(\partial_{y}^{\alpha} \phi_{0}^{(j)}(\rho ; .), \partial_{\eta}^{\alpha} \phi_{0}^{(r)}(\rho ; .)\right)_{L^{2}\left(\mathbb{R}^{\nu}\right)} \phi_{0}^{(r)}(\rho ; .), \\
\psi_{-1 / 2}^{\prime(j)}(\rho ; .)=\psi_{-1 / 2,2}^{(j)}(\rho ; .) \\
=E_{\rho}^{(-k)}\left[\sum_{r=1}^{d}\left(A_{\rho}^{(k+1)} \phi_{0}^{(j)}(\rho ; .), \phi_{0}^{(r)}(\rho ; .)\right)_{L^{2}\left(\mathbb{R}^{\nu}\right)} \phi_{0}^{(r)}(\rho ; .)-A_{\rho}^{(k+1)} \phi_{0}^{(j)}(\rho ; .)\right] .
\end{array}\right.
$$

\subsection{Example}

Consider in $\mathbb{R}^{2}$ the differential operator

$$
A=D_{x}^{2}+\mu^{2} x^{2} D_{y}^{2}+a(x, y) D_{x}+b(x, y) D_{y}+c(x, y),
$$

where $\mu>0$ is constant and $a, b, c \in C^{\infty}\left(\mathbb{R}^{2} ; \mathbb{C}\right)$. In this case, the localized operator $A_{\rho=(y, \eta)}^{(2)}$ is given by

$$
A_{(y, \eta)}^{(2)}=D_{x}^{2}+\mu^{2} x^{2} \eta^{2}+b(0, y) \eta
$$


Note that $\left[A_{(y, \eta)}^{(2)},\left(A_{(y, \eta)}^{(2)}\right)^{*}\right]=0$, because

$$
\left(A_{(y, \eta)}^{(2)}\right)^{*}=D_{x}^{2}+\mu^{2} \eta^{2} x^{2}+\overline{b(0, y)} \eta .
$$

As is well known,

$$
\operatorname{Spec}\left(A_{(y, \eta)}^{(2)}\right)=\left\{\lambda_{k}(y, \eta):=(2 k+1) \mu|\eta|+b(0, y) \eta ; k \in \mathbb{Z}_{+}\right\} .
$$

Each $\lambda_{k}$ has multiplicity 1 and relative normalized eigenfunction

$$
f_{k}(y, \eta ; x)=f_{k}(\eta ; x)=(\mu|\eta|)^{1 / 4} h_{k}(\sqrt{\mu|\eta|} x),
$$

where

$$
h_{k}(t)=\frac{1}{\pi^{1 / 4} \sqrt{2^{k} k !}}\left(\frac{d}{d t}-t\right)^{k}\left(e^{-t^{2} / 2}\right), \quad k \in \mathbb{Z}_{+},
$$

are the classical Hermite functions.

For definiteness, we study the hypoellipticity of $A$ at the origin $(0,0) \in \mathbb{R}^{2}$. From the Boutet-Grigis-Helffer Theorem 1.4 , we know that $A$ is hypoelliptic at $(0,0)$ with loss of one derivative if and only if

$$
b(0,0) \notin\left\{ \pm(2 k+1) \mu ; k \in \mathbb{Z}_{+}\right\} .
$$

We hence study the case in which, for some $j \in \mathbb{Z}_{+}$, one has

$$
b(0,0)= \pm(2 j+1) \mu .
$$

We treat two possible situations:

$(\mathrm{I})_{ \pm} b(0,0)= \pm(2 j+1) \mu$ and the function $y \longmapsto(2 j+1) \mu \mp b(0, y)$ vanishes exactly to order $r \geq 1$ at $y=0$.

(II) $)_{ \pm}$The function $y \longmapsto(2 j+1) \mu \mp b(0, y)$ vanishes identically near $y=0$ (for $|y|<\delta$, say).

\section{REMARK 6.1.}

1) Note that if $y \longmapsto b(0, y)$ is analytic near $y=0$ and $b(0,0)= \pm(2 j+1) \mu$, then $(I)_{ \pm}$and $(I I)_{ \pm}$are the only possibilities to be considered.

2) Note that if $b(0,0)= \pm(2 j+1) \mu$, then $A$ is already hypoelliptic at $(x=0$, $y=0, \xi=0, \pm \eta>0$ ) with loss of one derivative. We may hence microlocally construct the scalar operator $\Lambda\left(y, D_{y}\right) \in \mathrm{OPS}_{\mathrm{cl}}^{1}(\mathbb{R})$ in the conic region

$$
\Gamma_{\delta}^{\mp}:=\{(y, \eta) ;|y|<\delta, \pm \eta<0\} .
$$


We then take as the $\phi_{0}$ in the beginning of this section, the eigenfunction $f_{j}$. Since $f_{j}$ is independent of $y$, from (91) we immediately have that $\psi^{\prime}{ }_{-1,1} \equiv 0$. Moreover, since $A_{(y, \eta)}^{(3)}$ flips the parity, from (91) we have that

$$
\psi^{\prime}{ }_{-1 / 2}(y, \eta ; .)=-E_{(y, \eta)}^{(-2)} A_{(y, \eta)}^{(3)} f_{j}(\eta ; .) .
$$

As a consequence, taking again into account that $f_{j}$ is independent of $y$, formula (90), for $m=k=2$, reads as

$$
\left\{\begin{aligned}
\Lambda_{1}(y, \eta)= & \lambda_{j}(y, \eta), \\
\Lambda_{1 / 2}(y, \eta)= & 0 \\
\Lambda_{0}(y, \eta)= & \left(A_{(y, \eta)}^{(4)} f_{j}(\eta ; .), f_{j}(\eta ; .)\right)_{L^{2}(\mathbb{R})} \\
& \quad\left(E_{(y, \eta)}^{(-2)} A_{(y, \eta)}^{(3)} f_{j}(\eta ; .),\left(A_{(y, \eta)}^{(3)}\right)^{*} f_{j}(\eta ; .)\right)_{L^{2}(\mathbb{R})} \\
& +i \frac{\partial \lambda_{j}}{\partial y}(y, \eta)\left(\frac{\partial f_{j}}{\partial \eta}(\eta ; .), f_{j}(\eta ; .)\right)_{L^{2}(\mathbb{R})},
\end{aligned}\right.
$$

with $(y, \eta) \in \Gamma_{\delta}^{\mp}$, where

$$
\left\{\begin{array}{l}
A_{(y, \eta)}^{(3)}=a(0, y) D_{x}+\frac{\partial b}{\partial x}(0, y) x \eta, \\
A_{(y, \eta)}^{(4)}=\frac{\partial a}{\partial x}(0, y) x D_{x}+\frac{1}{2} \frac{\partial^{2} b}{\partial x^{2}}(0, y) x^{2} \eta+c(0, y) .
\end{array}\right.
$$

It is convenient to rewrite $A^{(3)}$ and $A^{(4)}$ in terms of the creation/annihilation operators

$$
\frac{d}{d x} \mp \mu|\eta| x,
$$

taking into account that, for all $k \in \mathbb{Z}_{+}$,

$$
\begin{gathered}
\left(\frac{d}{d x}-\mu|\eta| x\right) f_{k}(\eta ; x)=\sqrt{2(k+1) \mu|\eta|} f_{k+1}(\eta ; x), \\
\left(\frac{d}{d x}+\mu|\eta| x\right) f_{k}(\eta ; x)=-\sqrt{2 k \mu|\eta|} f_{k-1}(\eta ; x)
\end{gathered}
$$

(as usual, $f_{-1} \stackrel{\text { def }}{=} 0$ ). Hence, a computation gives

$$
A_{(y, \pm \eta<0)}^{(3)}=p\left(\frac{d}{d x}-\mu|\eta| x\right)-q \sqrt{\mu|\eta|} x,
$$

where

$$
p=\frac{1}{i} a(0, y), \quad q=i \sqrt{\mu|\eta|} a(0, y) \pm \sqrt{\frac{|\eta|}{\mu}} \frac{\partial b}{\partial x}(0, y),
$$


and

$$
A_{(y, \pm \eta<0)}^{(4)}=\alpha \sqrt{\mu|\eta|} x\left(\frac{d}{d x}-\mu|\eta| x\right)-\beta(\sqrt{\mu|\eta|} x)^{2}+\gamma
$$

where

$$
\alpha=\frac{1}{i \sqrt{\mu|\eta|}} \frac{\partial a}{\partial x}(0, y), \quad \beta=i \frac{\partial a}{\partial x}(0, y) \pm \frac{1}{2 \mu} \frac{\partial^{2} b}{\partial x^{2}}(0, y), \quad \gamma=c(0, y) .
$$

Note that

$$
\left(A_{(y, \pm \eta<0)}^{(3)}\right)^{*}=-\bar{p}\left(\frac{d}{d x}+\mu|\eta| x\right)-\bar{q} \sqrt{\mu|\eta|} x .
$$

We next compute $\Lambda_{0}(y, \pm \eta<0)$. We have

$$
\begin{aligned}
\left(\alpha \sqrt{\mu|\eta|} x\left(\frac{d}{d x}-\mu|\eta| x\right) f_{j}, f_{j}\right)_{L^{2}(\mathbb{R})} & =\alpha \sqrt{2(j+1) \mu|\eta|}\left(h_{j+1}, t h_{j}\right)_{L^{2}(\mathbb{R})} \\
& =-\alpha(j+1) \sqrt{\mu|\eta|},
\end{aligned}
$$

because

$$
t h_{j}=\frac{1}{2}\left(\frac{d}{d t}+t\right) h_{j}-\frac{1}{2}\left(\frac{d}{d t}-t\right) h_{j}=-\frac{1}{2}\left(\sqrt{2 j} h_{j-1}+\sqrt{2(j+1)} h_{j+1}\right) .
$$

Moreover,

$$
-\beta\left((\sqrt{\mu|\eta|} x)^{2} f_{j}, f_{j}\right)_{L^{2}(\mathbb{R})}=-\beta\left(t h_{j}, t h_{j}\right)_{L^{2}(\mathbb{R})}=-\frac{2 j+1}{2} \beta .
$$

In conclusion,

$$
\left(A_{(y, \pm \eta<0)}^{(4)} f_{j}, f_{j}\right)_{L^{2}(\mathbb{R})}=\gamma-\frac{2 j+1}{2} \beta-(j+1) \alpha \sqrt{\mu|\eta|} .
$$

On the other hand,

$-\left(E_{(y, \eta)}^{(-2)} A_{(y, \pm \eta<0)}^{(3)} f_{j}(\eta ; .),\left(A_{(y, \pm \eta<0)}^{(3)}\right)^{*} f_{j}(\eta ; .)\right)_{L^{2}(\mathbb{R})}$

$=-\sum_{k \neq j} \frac{1}{\lambda_{k}(y, \pm \eta<0)}\left(A_{(y, \pm \eta<0)}^{(3)} f_{j}(\eta ; .), f_{k}(\eta ; .)\right)_{L^{2}} \overline{\left(\left(A_{(y, \pm \eta<0)}^{(3)}\right)^{*} f_{j}(\eta ; .), f_{k}(\eta ; .)\right)_{L^{2}}}$.

Now

$$
\begin{aligned}
\left(A^{(3)} f_{j}, f_{k}\right)_{L^{2}} & =p\left(\left(\frac{d}{d x}-\mu|\eta| x\right) f_{j}, f_{k}\right)_{L^{2}}-q\left(\sqrt{\mu|\eta|} x f_{j}, f_{k}\right)_{L^{2}} \\
& =p \sqrt{2(j+1) \mu|\eta|} \delta_{j+1, k}-q\left(t h_{j}, h_{k}\right)_{L^{2}} \\
& =\left[p \sqrt{2(j+1) \mu|\eta|}+q \frac{\sqrt{2(j+1)}}{2}\right] \delta_{j+1, k}+q \frac{\sqrt{2 j}}{2} \delta_{j-1, k},
\end{aligned}
$$


and

$$
\begin{aligned}
\left(\left(A^{(3)}\right)^{*} f_{j}, f_{k}\right)_{L^{2}} & =-\bar{p}\left(\left(\frac{d}{d x}+\mu|\eta| x\right) f_{j}, f_{k}\right)_{L^{2}}-\bar{q}\left(\sqrt{\mu|\eta|} x f_{j}, f_{k}\right)_{L^{2}} \\
& =\bar{p} \sqrt{2 j \mu|\eta|} \delta_{j-1, k}-\bar{q}\left(t h_{j}, h_{k}\right)_{L^{2}} \\
& =\bar{q} \frac{\sqrt{2(j+1)}}{2} \delta_{j+1, k}+\left[\bar{p} \sqrt{2 j \mu|\eta|}+\bar{q} \frac{\sqrt{2 j}}{2}\right] \delta_{j-1, k} .
\end{aligned}
$$

Thus,

$$
\begin{gathered}
-\left(E^{(-2)} A^{(3)} f_{j},\left(A^{(3)}\right)^{*} f_{j}\right)_{L^{2}}=-\sum_{k \neq j} \frac{1}{\lambda_{k}}\left\{\left[p \sqrt{2(j+1) \mu|\eta|}+q \frac{\sqrt{2(j+1)}}{2}\right] \delta_{j+1, k}\right. \\
\left.+q \frac{\sqrt{2 j}}{2} \delta_{j-1, k}\right\}\left\{q \frac{\sqrt{2(j+1)}}{2} \delta_{j+1, k}+\left[p \sqrt{2 j \mu|\eta|}+q \frac{\sqrt{2 j}}{2}\right] \delta_{j-1, k}\right\} \\
=-\left(\frac{j+1}{\lambda_{j+1}}+\frac{j}{\lambda_{j-1}}\right)\left(\frac{q}{2}+p \sqrt{\mu|\eta|}\right) q .
\end{gathered}
$$

Finally, for $(y, \eta) \in \Gamma_{\delta}^{\mp}$, we have that

$$
\begin{aligned}
\Lambda_{1}(y, \eta)= & \lambda_{j}(y, \eta)=(2 j+1) \mu|\eta|+b(0, y) \eta, \\
\Lambda_{0}(y, \eta)= & \gamma-\frac{2 j+1}{2} \beta-(j+1) \sqrt{\mu|\eta|} \alpha \\
& -\left(\frac{j+1}{\lambda_{j+1}}+\frac{j}{\lambda_{j-1}}\right)\left(\frac{q}{2}+\sqrt{\mu|\eta|}\right) q \\
& +i \frac{\partial \lambda_{j}}{\partial y}(y, \eta)\left(\frac{\partial f_{j}}{\partial \eta}(\eta ; .), f_{j}(\eta ; .)\right)_{L^{2}(\mathbb{R})} .
\end{aligned}
$$

Since $f_{j}$ is real-valued and $\left\|f_{j}(\eta ; .)\right\|_{L^{2}}^{2}=1$, we have

$$
\left(\frac{\partial f_{j}}{\partial \eta}(\eta ; .), f_{j}(\eta ; .)\right)_{L^{2}(\mathbb{R})}=0
$$

so that the last term in $\Lambda_{0}$ vanishes.

Suppose now we are in situation (II) $)_{ \pm}$. Then $\lambda_{j}$ vanishes identically on $\Gamma_{\delta}^{\mp}$. As a consequence, if $\Lambda_{0}(y=0, \pm \eta<0) \neq 0$, then $\Lambda\left(y, D_{y}\right)$ is hypoelliptic at $(y=0, \pm \eta<0)$ with loss of one derivative. Now, since

$$
\lambda_{j+1}(0, \pm \eta<0)=2 \mu|\eta|, \quad \lambda_{j-1}(0, \pm \eta<0)=-2 \mu|\eta|,
$$


we obtain

$$
\begin{aligned}
\Lambda_{0}(y=0, \pm \eta<0)= & \gamma(0)-\frac{2 j+1}{2} \beta(0)-(j+1) \sqrt{\mu|\eta|} \alpha(0, \pm \eta<0) \\
& -\frac{1}{2 \mu|\eta|}\left(\frac{q(0, \pm \eta<0)}{2}+\sqrt{\mu|\eta|} p(0)\right) q(0, \pm \eta<0) .
\end{aligned}
$$

From (101) and (103), we have

$$
\begin{aligned}
\gamma(0) & -\frac{2 j+1}{2} \beta(0)-(j+1) \sqrt{\mu|\eta|} \alpha(0, \pm \eta<0) \\
& =c(0,0)+\frac{i}{2} \frac{\partial a}{\partial x}(0,0) \mp \frac{2 j+1}{4 \mu} \frac{\partial^{2} b}{\partial x^{2}}(0,0)
\end{aligned}
$$

and

$$
\begin{aligned}
-\frac{1}{2 \mu|\eta|} & \left(\frac{q(0, \pm \eta<0)}{2}+\sqrt{\mu|\eta|} p(0)\right) q(0, \pm \eta<0) \\
= & -\frac{1}{2 \mu|\eta|}\left(\frac{i}{2} \sqrt{\mu|\eta|} a(0,0) \pm \frac{1}{2} \sqrt{\frac{|\eta|}{\mu}} \frac{\partial b}{\partial x}(0,0)-i \sqrt{\mu|\eta|} a(0,0)\right) \\
& \times\left(i \sqrt{\mu|\eta|} a(0,0) \pm \sqrt{\frac{|\eta|}{\mu}} \frac{\partial b}{\partial x}(0,0)\right) \\
= & \frac{1}{4}\left(i a(0,0) \mp \frac{1}{\mu} \frac{\partial b}{\partial x}(0,0)\right)\left(i a(0,0) \pm \frac{1}{\mu} \frac{\partial b}{\partial x}(0,0)\right) \\
= & -\frac{1}{4}\left(a(0,0)^{2}+\frac{1}{\mu^{2}} \frac{\partial b}{\partial x}(0,0)^{2}\right) .
\end{aligned}
$$

At last, when (II) $)_{ \pm}$holds, we have

$$
\begin{aligned}
\Lambda_{0}(0, \pm \eta<0)= & c(0,0)+\frac{i}{2} \frac{\partial a}{\partial x}(0,0) \\
& \mp \frac{2 j+1}{4 \mu} \frac{\partial^{2} b}{\partial x^{2}}(0,0)-\frac{1}{4}\left(a(0,0)^{2}+\frac{1}{\mu^{2}} \frac{\partial b}{\partial x}(0,0)^{2}\right)=: T_{ \pm} .
\end{aligned}
$$

We have finally proved the following proposition.

Proposition 6.2. If $(I I)_{ \pm}$holds and $T_{ \pm} \neq 0$, then $A$ is hypoelliptic at $(0,0)$ with loss of two derivatives.

We next pass to considering the case in which $(\mathrm{I})_{ \pm}$holds. There are two quite different possibilities: 
(a) $\quad b(0,0)= \pm(2 j+1) \mu$ and $\left(d \lambda_{j} / d y\right)(0, \pm \eta<0) \neq 0$

(b) $\quad\left(d^{\ell} \lambda_{j} / d y^{\ell}\right)(0, \pm \eta<0)=0, \ell=0,1, \ldots, r-1$, and $\left(d^{r} \lambda_{j} / d y^{r}\right)(0, \pm \eta<$ $0) \neq 0$ for some $r \geq 2$.

In any event, note that

$$
\Lambda_{1}(y, \eta)=\theta_{ \pm}(y) y^{r}|\eta|
$$

where $\theta_{ \pm}$is smooth near $y=0$ and

$$
\theta_{ \pm}(0)=\frac{1}{r !} \frac{d^{r} \lambda_{j}}{d y^{r}}(0, \mp 1) \neq 0 .
$$

Thanks to classical results on principal type operators (see [10, Vol. IV, Theorem 26.1.1]) we have the following proposition.

Proposition 6.3. Suppose $(I)_{ \pm}$and (a) hold. Then $A$ is not hypoelliptic at $(0,0)$. In fact, one may find a distribution $u_{ \pm} \in \mathcal{D}^{\prime}\left(\mathbb{R}^{2}\right)$ such that $A u_{ \pm} \in C^{\infty}$ at $(0,0)$ and $(x=0, y=0, \xi=0, \pm \eta<0) \in \mathrm{WF}\left(u_{ \pm}\right)$.

When (b) holds, the situation can be much more complicated. In particular, $\Lambda\left(y, D_{y}\right)$ can still be hypoelliptic at $(y=0, \pm \eta<0)$ with loss of one derivative, as the following proposition shows.

Proposition 6.4. Suppose $(I)_{ \pm}$and $(b)$ hold and that the following conditions are satisfied

$$
\left\{\begin{array}{l}
\tau+\frac{T_{ \pm}}{\theta_{ \pm}(0)} \neq 0, \quad \text { for all } \tau \geq 0, \\
\tau+\frac{T_{ \pm}}{\theta_{ \pm}(0)} \neq 0, \quad \text { for all } \tau \in \mathbb{R}, \quad \text { when } r \text { is even } \text { is odd } .
\end{array}\right.
$$

Then $A$ is hypoelliptic at $(0,0)$ with loss of two derivatives.

Proof. The symbol of $\left(1 / \theta_{ \pm}(y)\right) \Lambda\left(y, D_{y}\right)$ is, modulo $S^{-1 / 2}$, of the form

$$
y^{r}|\eta|+\frac{1}{\theta_{ \pm}(y)} \Lambda_{0}(y, \pm \eta<0)
$$

The hypothesis ensures that

$$
\left|y^{r}\right| \eta\left|+\frac{T_{ \pm}}{\theta_{ \pm}(0)}\right| \geq \text { constant }>0, \quad \text { for all } y \in \mathbb{R}, \quad \text { for all } \eta \neq 0 .
$$

Since $\Lambda_{0}(0, \pm \eta<0)=T_{ \pm}$, there exist $0<\delta^{\prime}<\delta$ sufficiently small and $c>0$ such that, with

$$
\sigma\left(\frac{1}{\theta_{ \pm}(y)} \Lambda\left(y, D_{y}\right)\right)=: g(y, \eta)
$$


we have

$$
|g(y, \eta)| \geq c, \quad \text { for all } y,|y| \leq \delta^{\prime}, \text { for all } \eta, \pm \eta<0,|\eta| \geq \frac{1}{\delta^{\prime}} .
$$

On the other hand, it is easy to show that for all $p, q \in \mathbb{Z}_{+}$,

$$
\left|\frac{\partial_{y}^{p} \partial_{\eta}^{q} g(y, \eta)}{g(y, \eta)}\right| \leq C_{p q}|\eta|^{-q+p / r}, \quad \text { for all } y,|y| \leq \delta^{\prime}, \text { for all } \eta, \pm \eta<0,|\eta| \geq \frac{1}{\delta^{\prime}}
$$

As a consequence, $\Lambda\left(y, D_{y}\right)$ has a microlocal two-sided parametrix in the (microlocal) Hörmander class $\operatorname{OPS}_{1,1 / r}^{0}(\mathbb{R})$, which ensures the hypoellipticity of $\Lambda\left(y, D_{y}\right)$ at $(y=0, \pm \eta<0)$ with loss of one derivative and, hence, that of $A$ at $(0,0)$ with loss of two derivatives.

REMARK 6.5.

1) Note that if

$$
a(0,0)=\frac{\partial a}{\partial x}(0,0)=\frac{\partial b}{\partial x}(0,0)=\frac{\partial^{2} b}{\partial x^{2}}(0,0)=0,
$$

then $T_{ \pm}=c(0,0)$, so that in Proposition 6.2 there is hypoellipticity whenever $c(0,0) \neq 0$.

2) In Proposition 6.4, the hypothesis is obviously satisfied if $\operatorname{Im}\left(T_{ \pm} / \theta_{ \pm}(0)\right) \neq 0$.

When $T_{ \pm}=0$, the hypoellipticity or non-hypoellipticity of $\Lambda\left(y, D_{y}\right)$ is decided by a precise knowledge of the terms $\Lambda_{-1 / 2}, \Lambda_{-1}, \ldots$

REMARK 6.6. We can consider the seemingly more general case of an operator in $\mathbb{R}^{2}$

$$
A^{\prime}=D_{x^{\prime}}^{2}+2 \alpha x^{\prime} D_{x^{\prime}} D_{y^{\prime}}+\beta x^{\prime 2} D_{y^{\prime}}^{2}+p\left(x^{\prime}, y^{\prime}\right) D_{x^{\prime}}+q\left(x^{\prime}, y^{\prime}\right) D_{y^{\prime}}+r\left(x^{\prime}, y^{\prime}\right),
$$

$p, q, r \in C^{\infty}\left(\mathbb{R}^{2} ; \mathbb{C}\right), \alpha, \beta \in \mathbb{R}$ with $\beta>\alpha^{2}$.

In fact, by the change of variables $x=x^{\prime}, y=y^{\prime}-\alpha x^{\prime 2} / 2, A^{\prime}$ goes over to the operator A defined in (92), with $\mu^{2}=\beta-\alpha^{2}$ and

$$
\left\{\begin{array}{l}
a(x, y)=p\left(x, y+\alpha x^{2} / 2\right) \\
b(x, y)=q\left(x, y+\alpha x^{2} / 2\right)-x \alpha p\left(x, y+\alpha x^{2} / 2\right)+i \alpha \\
c(x, y)=r\left(x, y+\alpha x^{2} / 2\right)
\end{array}\right.
$$

\subsection{Example}

Consider in $\mathbb{R}^{n}=\mathbb{R}_{x} \times \mathbb{R}_{y}^{n-1}(n \geq 3)$ the following fourth-order differential operator

$$
A=M^{2}+a D_{x}^{2}+\left\langle b, D_{y}\right\rangle D_{x}+\left\langle c D_{y}, D_{y}\right\rangle+\alpha D_{x}+\left\langle\beta, D_{y}\right\rangle+\gamma,
$$


where

$$
M=D_{x}^{2}+\mu^{2} x^{2}\left|D_{y}\right|^{2}, \quad \mu>0,
$$

$a, \alpha, \gamma \in C^{\infty}\left(\mathbb{R}^{n} ; \mathbb{C}\right), b, \beta \in C^{\infty}\left(\mathbb{R}^{n} ; \mathbb{C}^{n-1}\right), c={ }^{t} c$ is a smooth $(n-1) \times(n-1)$ symmetric complex matrix.

Again, we consider the hypoellipticity of $A$ at the origin $(0,0)$. The localized operator $A_{(y, \eta)}^{(4)}$ is

$$
A_{(y, \eta)}^{(4)}=\left(D_{x}^{2}+\mu^{2} x^{2}|\eta|^{2}\right)^{2}+\langle c(0, y) \eta, \eta\rangle
$$

For simplicity, suppose that $c(0, y)$ is self-adjoint, so that $A_{(y, \eta)}^{(4)}=\left(A_{(y, \eta)}^{(4)}\right)^{*}$. Of course,

$$
\operatorname{Spec}\left(A_{(y, \eta)}^{(4)}\right)=\left\{\lambda_{k}(y, \eta) ; k \in \mathbb{Z}_{+}\right\}
$$

where

$$
\lambda_{k}(y, \eta)=\left\langle q_{k}(y) \eta, \eta\right\rangle, \quad q_{k}(y):=c(0, y)+(2 k+1)^{2} \mu^{2} I_{n-1} .
$$

The $\lambda_{k}$ have multiplicity 1 and the corresponding normalized eigenfunctions are the $f_{k}(\eta ; x)$ defined in (94) (now $\left.\eta \in \mathbb{R}^{n-1} \backslash\{0\}\right)$.

Put

$$
I:=\left\{k \in \mathbb{Z}_{+} ; q_{k}(0) \text { is either positive-definite or negative-definite }\right\}
$$

and let

$$
J:=\mathbb{Z}_{+} \backslash I
$$

Note that $J$ is a finite, possibly empty, set. The Boutet-Grigis-Helffer Theorem 1.4 then states that $A$ is hypoelliptic at $(0,0)$ with loss of two derivatives if and only if $J=\emptyset$ (note that $\mathbb{R}_{\eta}^{n-1} \backslash\{0\}$ is connected).

Suppose now $J \neq \emptyset$. There is a case which is trivial to handle, namely when, for some $k \in J$ and some $\eta_{0} \neq 0$, we have

$$
\lambda_{k}\left(0, \eta_{0}\right)=0, \quad \nabla_{\eta} \lambda_{k}\left(0, \eta_{0}\right)=2 q_{k}(0) \eta_{0} \neq 0 .
$$

If this happens, we have the following result.

Proposition 6.7. Suppose (120) holds. Then A is not hypoelliptic at $(0,0)$. Furthermore, upon denoting by $\psi(t)=(y(t), \eta(t)), t \in \mathbb{R}$, the integral curves of $H_{\lambda_{k}}=\left(\nabla_{\eta} \lambda_{k},-\nabla_{y} \lambda_{k}\right)$ with $\psi(0)=\left(0, \eta_{0}\right)$, then for any given $u \in \mathcal{D}^{\prime}\left(\mathbb{R}^{n}\right)$ for which

$$
\left(x=0, y=0, \xi=0, \eta=\eta_{0}\right) \in \mathrm{WF}(u) \backslash \mathrm{WF}(A u),
$$


one has

$$
(x=0, y=y(t), \xi=0, \eta=\eta(t)) \in \mathrm{WF}(u) \backslash \mathrm{WF}(A u),
$$

for all t sufficiently small.

Proof. For all $k^{\prime} \neq k$, we have $\lambda_{k^{\prime}}\left(0, \eta_{0}\right) \neq 0$, for

$$
\lambda_{k}(y, \eta)-\lambda_{k^{\prime}}(y, \eta)=\left((2 k+1)^{2}-\left(2 k^{\prime}+1\right)^{2}\right) \mu^{2}|\eta|^{2} .
$$

We may, hence, find a conic neighborhood $\Gamma$ of $\left(0, \eta_{0}\right)$ on which only $\lambda_{k}$ vanishes and $H_{\lambda_{k}} \neq 0$. The corresponding operator $\Lambda\left(y, D_{y}\right) \in \mathrm{OPS}_{\mathrm{cl}}^{2}\left(\mathbb{R}^{n-1}\right)$ is, therefore, of real principal type in $\Gamma$ by virtue of the fact that $\Lambda_{3 / 2}(y, \eta)=0$ on $\Gamma$. Once more, the conclusion follows from Theorem 26.1.1 in Hörmander's book [10, Vol. IV], and Theorem 5.2.

Note that a possible example is $\lambda_{k}(0, \eta)=\eta_{1}^{2}-\eta_{2}^{2}$ in $\mathbb{R}_{\eta}^{3}$.

We are, thus, left with considering the case in which the functions $0 \neq \eta \longmapsto$ $\lambda_{k}(0, \eta), k \in J$, have only zeros of multiplicity 2 , i.e. we may suppose

$$
k \in J \Longrightarrow \begin{cases}q_{k}(0) & \text { is either positive-semidefinite or } \\ \operatorname{Ker} q_{k}(0) \neq\{0\} . & \text { negative-semidefinite }\end{cases}
$$

Hence,

$$
\text { either } J=\{k\} \quad \text { or } J=\{k, k+1\}, \quad \text { for some } k \in \mathbb{Z}_{+},
$$

and, in the latter case, we necessarily have, by (121),

$$
q_{k}(0) \leq 0, \quad q_{k+1}(0) \geq 0 .
$$

It is important to observe that the sole semi-definiteness of $q_{k}(0)$ does not give sufficient information on the zeros of $\lambda_{k}(y, \eta)$ for $y$ near 0 .

To focus on one possible case, let us assume that there exists a neighborhood $U \subset \mathbb{R}^{n-1}$ of the origin and, for any given $k \in J$ a smooth submanifold $\Sigma_{k} \subset$ $U \times\left(\mathbb{R}^{n-1} \backslash\{0\}\right)$ such that $\lambda_{k}(y, \eta),(y, \eta) \in U \times\left(\mathbb{R}^{n-1} \backslash\{0\}\right)$ vanishes exactly to second order on $\Sigma_{k}$.

A crucial observation is that, by possibly shrinking $U$, we may suppose $\Sigma_{k^{\prime}} \cap$ $\Sigma_{k}=\emptyset$ when $k \neq k^{\prime}$ (again by virtue of (121)). Hence, we are reduced to studying the hypoellipticity of $A$ at a point $\left(x=0, y=0, \xi=0, \eta=\eta_{0}\right)$ with $\left(0, \eta_{0}\right) \in \Sigma_{k}$ for a unique $k \in J$. One can, therefore, find a conic neighborhood 
$\Gamma \subset T^{*} \mathbb{R}^{n-1} \backslash 0$ of $\left(0, \eta_{0}\right)$ where only $\lambda_{k}$ vanishes. Once again, we microlocally construct $\Lambda\left(y, D_{y}\right) \in \operatorname{OPS}_{\mathrm{cl}}^{2}\left(\mathbb{R}^{n-1}\right)$ with (now $m=k=4$ )

$$
\left\{\begin{aligned}
\Lambda_{2}(y, \eta)= & \lambda_{k}(y, \eta) \\
\Lambda_{3 / 2}(y, \eta)= & 0, \\
\Lambda_{1}(y, \eta)= & \left.A_{(y, \eta)}^{(6)} f_{k}(\eta ; .), f_{k}(\eta ; .)\right)_{L^{2}(\mathbb{R})} \\
& \quad-\left(E_{(y, \eta)}^{(-4)} A_{(y, \eta)}^{(5)} f_{k}(\eta ; .),\left(A_{(y, \eta)}^{(5)}\right)^{*} f_{k}(\eta ; .)\right)_{L^{2}(\mathbb{R})} \\
& +i \sum_{j=1}^{n-1} \frac{\partial \lambda_{k}}{\partial y_{j}}(y, \eta)\left(\frac{\partial f_{k}}{\partial \eta_{j}}(\eta ; .), f_{k}(\eta ; .)\right)_{L^{2}(\mathbb{R})} \\
& +\frac{1}{i} \sum_{j=1}^{n-1}\left(\frac{\partial A_{(y, \eta)}^{(4)}}{\partial \eta_{j}} \frac{\partial f_{k}}{\partial y_{j}}(\eta ; .), f_{k}(\eta ; .)\right)_{L^{2}(\mathbb{R})}
\end{aligned}\right.
$$

where

$$
\begin{aligned}
& A_{(y, \eta)}^{(5)}=\langle b(0, y), \eta\rangle D_{x}+\left\langle\frac{\partial c}{\partial x}(0, y) \eta, \eta\right\rangle x \\
& =\ell(y, \eta)\left(\frac{d}{d x}-\mu|\eta| x\right)+m(y, \eta) \sqrt{\mu|\eta|} x, \\
& \left\{\begin{array}{l}
\ell(y, \eta)=\frac{1}{i}\langle b(0, y), \eta\rangle, \\
m(x, y)=\frac{1}{i}\langle b(0, y), \eta\rangle \sqrt{\mu|\eta|}+\frac{1}{\sqrt{\mu|\eta|}}\left\langle\frac{\partial c}{\partial x}(0, y) \eta, \eta\right\rangle,
\end{array}\right. \\
& A_{(y, \eta)}^{(6)}=a(0, y) D_{x}^{2}+\left\langle\frac{\partial b}{\partial x}(0, y), \eta\right\rangle x D_{x}+\frac{1}{2}\left\langle\frac{\partial^{2} c}{\partial x^{2}}(0, y) \eta, \eta\right\rangle x^{2}+\langle\beta(0, y), \eta\rangle \\
& =P(y)\left(\frac{d}{d x}-\mu|\eta| x\right)^{2}+Q(y, \eta) \sqrt{\mu|\eta|} x\left(\frac{d}{d x}-\mu|\eta| x\right) \\
& +R(y, \eta)(\sqrt{\mu|\eta|} x)^{2}+S(y, \eta), \\
& \left\{\begin{array}{l}
P(y)=-a(0, y) \\
Q(y, \eta)=\frac{1}{i \sqrt{\mu|\eta|}}\left\langle\frac{\partial b}{\partial x}(0, y), \eta\right\rangle-2 a(0, y) \sqrt{\mu|\eta|}
\end{array}\right. \\
& \left\{\begin{array}{l}
R(y, \eta)=-a(0, y) \mu|\eta|+\frac{1}{i}\left\langle\frac{\partial b}{\partial x}(0, y), \eta\right\rangle+\frac{1}{2 \mu|\eta|}\left\langle\frac{\partial^{2} c}{\partial x^{2}}(0, y) \eta, \eta\right\rangle, \\
S(y, \eta)=\langle\beta(0, y), \eta\rangle-a(0, y) \mu|\eta| .
\end{array}\right.
\end{aligned}
$$


Note that exactly as in Example 6.1, we have

$$
\left(\frac{\partial f_{k}}{\partial \eta_{j}}, f_{k}\right)_{L^{2}(\mathbb{R})}=0, \quad 1 \leq j \leq n-1,
$$

and that the last term in $\Lambda_{1}$ vanishes for $f_{k}$ does not depend on $y$.

By analogous computations as in Example 6.1, we obtain

$$
\begin{aligned}
\Lambda_{1}(y, \eta)= & \left(\frac{k}{\lambda_{k-1}(y, \eta)}+\frac{k+1}{\lambda_{k+1}(y, \eta)}\right)\left(\sqrt{\mu|\eta|} m(y, \eta) \ell(y, \eta)-\frac{m(y, \eta)^{2}}{2}\right) \\
& -(k+1) \sqrt{\mu|\eta|} Q(y, \eta)+\frac{2 k+1}{2} R(y, \eta)+S(y, \eta) .
\end{aligned}
$$

To decide the hypoellipticity of $\Lambda\left(y, D_{y}\right)$ with loss of one derivative at $(y=0, \eta=$ $\eta_{0}$ ), we just need to apply Theorem 22.4 .15 of Hörmander [10, Vol. III], bearing in mind that

$$
\text { either } \lambda_{k}(y, \eta) \geq 0 \text { or } \quad \lambda_{k}(y, \eta) \leq 0,
$$

and, in all cases, it vanishes exactly to second order on $\Sigma_{k}$.

Put

$$
\operatorname{Spec}\left(F_{\lambda_{k}}\left(0, \eta_{0}\right)\right) \backslash\{0\}=\left\{ \pm i \theta_{\ell} ; \theta_{\ell}>0, \ell=1, \ldots, r\right\}
$$

$(r \geq 0)$. Then the hypoellipticity condition reads as

$$
\Lambda_{1}^{s}\left(0, \eta_{0}\right) \pm \sum_{\ell=1}^{r}\left(2 h_{\ell}+1\right) \theta_{\ell}+\sigma\left(v, F_{\lambda_{k}}\left(0, \eta_{0}\right) v\right) \neq 0
$$

for all $h \in \mathbb{Z}_{+}^{r}$, for all $v \in \operatorname{Ker}\left(F_{\lambda_{k}}\left(0, \eta_{0}\right)^{2}\right), \Lambda_{1}^{s}$ being the subprincipal symbol of $\Lambda$, and $\sigma(.,$.$) the canonical symplectic form. In (129), we take +\sum\left(2 h_{\ell}+1\right) \theta_{\ell}$, respectively $-\sum\left(2 h_{\ell}+1\right) \theta_{\ell}$, according to whether $\lambda_{k} \geq 0$ or $\lambda_{k} \leq 0$, respectively.

REMARK 6.8. We have supposed that $\lambda_{k}(y, \eta), k \in J$, vanishes exactly to second order on some submanifold $\Sigma_{k}$. Of course, there are many more different possibilities. We put in evidence a few of them.

1) $\quad \lambda_{k}(y, \eta)=0$ identically for $y$ near 0 . As in Example 6.1, one has that $\Lambda\left(y, D_{y}\right)$ is hypoelliptic at $y=0$ with loss of one derivative provided that $\Lambda_{1}(0, \eta) \neq 0$ for all $\eta \neq 0$.

2) For y near $0, \lambda_{k}(y, \eta)=|y|^{2 p}\langle\Phi(y) \eta, \eta\rangle$, for some integer $p \geq 1$ and some smooth matrix $\Phi(y)=\Phi(y)^{*}>0$.

In this case, the condition

$$
\Lambda_{1}\left(0, \frac{\eta}{|\eta|}\right)+\tau \neq 0, \text { for all } \eta \neq 0, \text { for all } \tau \geq 0,
$$


implies that $\Lambda\left(y, D_{y}\right)$ has a microlocal parametrix in $\mathrm{OPS}_{1, \frac{1}{2 p}}^{-1}\left(\mathbb{R}^{n-1}\right)$, which again yields the hypoellipticity of $\Lambda$ with loss of one derivative. (The proof goes as in the corresponding case of Example 6.1.)

3) For y near 0 ,

$$
\text { either } \lambda_{k}(y, \eta)=\eta_{1}^{2}-y_{1}^{2 p}\left|\eta^{\prime}\right|^{2} \quad \text { or } \quad \lambda_{k}(y, \eta)=\eta_{1}^{2}-y_{1}^{2 p-1}\left|\eta^{\prime}\right|^{2}
$$

$\eta^{\prime}=\left(\eta_{2}, \ldots, \eta_{n-1}\right)$, for some integer $p \geq 1$. In either case, we have that $\Lambda\left(y, D_{y}\right)$, and therefore $A$, is not hypoelliptic at 0 and a propagation of singularities occurs in the characteristic set of A (see Ivrii [11] and Hörmander [10, Vol. III, Section 23.4]).

\subsection{Example}

In the previous examples, the characteristic manifold $\Sigma$ had codimension 2. We now wish to treat Grushin-type operators with a $\Sigma$ of codimension greater than two. In this case $\Lambda\left(y, D_{y}\right)$ can be a genuine system.

Let $n \geq 4,2 \leq v<n$ and let $Y \subset \mathbb{R}^{n-v}$ be open. On $\mathbb{R}^{v} \times Y$, consider the following operator

$A=\sum_{j, k=1}^{v}\left[P_{j k}\left(y, D_{y}\right) x_{j} x_{k}+Q_{j k}\left(y, D_{y}\right) x_{k} D_{x_{j}}+R_{j k}\left(y, D_{y}\right) D_{x_{k}} D_{x_{j}}\right]+T\left(y, D_{y}\right)$,

where $P_{j k}=P_{k j}, R_{j k}=R_{k j}, Q_{j k}$ and $T$ are linear differential operators in $Y$, with smooth coefficients, of order $m, m-2, m-1, m-1$, respectively.

For $(y, \eta) \in T^{*} Y \backslash 0$, denote by $p(y, \eta), r(y, \eta), q(y, \eta)$, respectively, the $v \times v$ matrices of the principal symbols of $P_{j k}, R_{j k}$ and $Q_{j k}$, respectively, and likewise denote by $t(y, \eta)$ the principal symbol of $T$. Note that these matrices are invariantly defined on $T^{*} Y$.

We suppose that $p, r, q$ are all real matrices and that the quadratic form

$$
\left\langle\left[\begin{array}{cc}
p(y, \eta) & \frac{1}{2}^{t} q(y, \eta) \\
\frac{1}{2} q(y, \eta) & r(y, \eta)
\end{array}\right]\left[\begin{array}{l}
x \\
\xi
\end{array}\right],\left[\begin{array}{l}
x \\
\xi
\end{array}\right]\right\rangle_{\mathbb{R}^{2 v}}
$$

is positive definite for all $(y, \eta) \in T^{*} Y \backslash 0$.

As a consequence, the principal symbol $a(x, y, \xi, \eta)$ of $A$ satisfies

$$
a(x, y, \xi, \eta) \lesssim|\eta|^{m}\left(|x|^{2}+\frac{|\xi|^{2}}{|\eta|^{2}}\right) \quad \text { and }|\eta|^{m}\left(|x|^{2}+\frac{|\xi|^{2}}{|\eta|^{2}}\right) \lesssim a(x, y, \xi, \eta)
$$


so that the characteristic manifold $\Sigma=\left\{(x, y, \xi, \eta) \in T^{*}\left(\mathbb{R}^{v} \times Y\right) \backslash 0 ; x=\xi=0\right\}$ can be identified with $T^{*} Y \backslash 0$.

For $(y, \eta) \in T^{*} Y \backslash 0$,

$A_{(y, \eta)}^{(2)}\left(x, D_{x}\right)=\sum_{j, k=1}^{\nu}\left[p_{j k}(y, \eta) x_{j} x_{k}+q_{j k}(y, \eta) x_{k} D_{x_{j}}+r_{j k}(y, \eta) D_{x_{k}} D_{x_{j}}\right]+t(y, \eta)$.

The problem is to determine the spectrum of $A_{(y, \eta)}^{(2)}$ as an unbounded operator in $L^{2}\left(\mathbb{R}^{v}\right)$ (with domain $B^{2}\left(\mathbb{R}^{v}\right)$ ). To this end, consider the fundamental matrix $F(y, \eta)$ restricted to $\mathbb{C} \otimes T_{(y, \eta)} \Sigma^{\sigma} \simeq \mathbb{C}^{2 v}$ :

$$
F(y, \eta):=\left[\begin{array}{cc}
\frac{1}{2} q(y, \eta) & r(y, \eta) \\
-p(y, \eta) & -\frac{1}{2}^{t} q(y, \eta)
\end{array}\right]: \mathbb{C}^{2 v} \longrightarrow \mathbb{C}^{2 v} .
$$

It is well known that

$$
\operatorname{Spec}(F(y, \eta))=\left\{ \pm i \mu_{1}(y, \eta), \ldots, \pm i \mu_{\nu}(y, \eta)\right\},
$$

for some $\mu_{j}(y, \eta)>0, \mu_{j}(y, t \eta)=t^{m-1} \mu_{j}(y, \eta), j=1, \ldots, v$, for all $(y, \eta) \in$ $T^{*} Y \backslash 0$, for all $t>0$.

We make the following assumption

$$
\left\{\begin{array}{l}
A_{(y, \eta)}^{(2)}=\left(A_{(y, \eta)}^{(2)}\right)^{*}, \\
\quad \text { i.e. } a^{\prime}(y, \eta):=t(y, \eta)+\frac{i}{2} \operatorname{Tr} q(y, \eta) \in \mathbb{R}, \quad \text { for all }(y, \eta) \in T^{*} Y \backslash 0, \\
\mu_{j} \in C^{\infty}\left(T^{*} Y \backslash 0 ; \mathbb{R}_{+}\right), j=1, \ldots, \nu .
\end{array}\right.
$$

We, therefore, have (see [9]) that

$$
\operatorname{Spec}\left(A_{(y, \eta)}^{(2)}\right)=\left\{\gamma_{(y, \eta)}(\alpha) ; \alpha \in \mathbb{Z}_{+}^{v}\right\},
$$

with

$$
\gamma_{(y, \eta)}(\alpha)=2\langle\alpha, \mu(y, \eta)\rangle_{\mathbb{R}^{v}}+\operatorname{Tr}^{+} F(y, \eta)+a^{\prime}(y, \eta) .
$$

Let $\rho_{0}=\left(y_{0}, \eta_{0}\right) \in T^{*} Y \backslash 0$ and suppose that

$$
J:=\left\{\alpha \in \mathbb{Z}_{+}^{v} ; \gamma_{\rho_{0}}(\alpha)=0\right\} \text { has } \operatorname{card}(J)=d \geq 1
$$

(if $d>1$, the $\mu_{j}\left(\rho_{0}\right), 1 \leq j \leq d$, are necessarily rationally dependent).

We further assume that there exist

- $\quad$ a conic neighborhood $\Gamma \subset T^{*} Y \backslash 0$ of $\rho_{0}$; 
- $\quad$ functions $\psi_{1}, \ldots, \psi_{d} \in C^{\infty}\left(\Gamma ; \mathcal{S}\left(\mathbb{R}^{v}\right)\right)$ such that

$$
\begin{gathered}
\psi_{j}\left(y, t \eta ; t^{-1 / 2} x\right)=t^{\nu / 4} \psi_{j}(y, \eta ; x), \\
\left(\psi_{j}(y, \eta ; .), \psi_{j^{\prime}}(y, \eta ; .)\right)_{L^{2}\left(\mathbb{R}^{\nu}\right)}=\delta_{j j^{\prime}},
\end{gathered}
$$

for all $1 \leq j, j^{\prime} \leq d,(y, \eta) \in \Gamma, t>0, x \in \mathbb{R}^{v}$;

- $\quad$ a bijection $\theta:\{1,2, \ldots, d\} \longrightarrow J$ such that

$$
A_{(y, \eta)}^{(2)} \psi_{j}(y, \eta ; .)=\lambda_{j}(y, \eta) \psi_{j}(y, \eta ; .), \quad j=1, \ldots, d,(y, \eta) \in \Gamma,
$$

with

$$
\lambda_{j}(y, \eta):=\gamma_{(y, \eta)}(\theta(j))
$$

- a smooth symplectic submanifold $\Sigma^{\prime} \subset \Gamma$ of codimension $2 v^{\prime}, 1 \leq v^{\prime}<n-v$, $\rho_{0} \in \Sigma^{\prime}$, such that every $\lambda_{j}$ is either $\geq 0$ or $\leq 0$ in $\Gamma$, and vanishes exactly to second order on $\Sigma^{\prime}$.

For $(y, \eta) \in \Sigma^{\prime}$, let $F_{j}(y, \eta): \mathbb{C} \otimes\left(T_{(y, \eta)} \Sigma^{\prime}\right)^{\sigma} \longrightarrow \mathbb{C} \otimes\left(T_{(y, \eta)} \Sigma^{\prime}\right)^{\sigma}, 1 \leq j \leq d$, be the fundamental matrix of $\lambda_{j}$ at $(y, \eta), j=1, \ldots, d$. Put

$$
\operatorname{Spec}\left(F_{j}(y, \eta)\right)=\left\{ \pm i h_{1}^{(j)}(y, \eta), \ldots, \pm i h_{v^{\prime}}^{(j)}(y, \eta)\right\},
$$

where $h_{\ell}^{(j)}(y, \eta)>0, \ell=1, \ldots, v^{\prime}$, and define for $(y, \eta) \in \Sigma^{\prime}$ and $\beta=$ $\left(\beta^{(1)}, \ldots, \beta^{(d)}\right) \in\left(\mathbb{Z}_{+}^{v^{\prime}}\right)^{d}$, the matrix

$$
\Delta_{\beta}(y, \eta)=\operatorname{diag}\left(\epsilon_{j}\left\langle\beta^{(j)}, h^{(j)}(y, \eta)\right\rangle_{\mathbb{R}^{v^{\prime}}}+\epsilon_{j} \operatorname{Tr}^{+} F_{j}(y, \eta)\right)_{j=1, \ldots, d},
$$

where $\epsilon_{j}=1$, respectively $\epsilon_{j}=-1$, if $\lambda_{j} \geq 0$, respectively $\lambda_{j} \leq 0$.

Our purpose is now to prove the following result.

PROPOSITION 6.9. In the above hypotheses, there exists a smooth $d \times d$ matrix $\gamma(y, \eta),(y, \eta) \in \Sigma^{\prime}$ (see (138) below), such that $A$ is hypoelliptic at $\rho_{0}$ with loss of two derivatives if and only if for all $\beta=\left(\beta^{(1)}, \ldots, \beta^{(d)}\right) \in\left(\mathbb{Z}_{+}^{v^{\prime}}\right)^{d}$ the matrix

$$
\Delta_{\beta}\left(\rho_{0}\right)+\gamma\left(\rho_{0}\right)
$$

is invertible.

Proof. We start out by observing that

$$
\begin{aligned}
\sigma_{m-1}(A)(x, y, \xi, \eta)= & \left\langle\sigma_{m-1}(P)(y, \eta) x, x\right\rangle+\left\langle\sigma_{m-2}(Q)(y, \eta) x, \xi\right\rangle \\
& +\left\langle\sigma_{m-3}(R)(y, \eta) \xi, \xi\right\rangle+t(y, \eta), \\
\sigma_{m-2}(A)(x, y, \xi, \eta)= & \left\langle\sigma_{m-2}(P)(y, \eta) x, x\right\rangle+\left\langle\sigma_{m-3}(Q)(y, \eta) x, \xi\right\rangle \\
& +\left\langle\sigma_{m-4}(R)(y, \eta) \xi, \xi\right\rangle+\sigma_{m-2}(T)(y, \eta),
\end{aligned}
$$

$(x, \xi) \in T^{*} \mathbb{R}^{v},(y, \eta) \in T^{*} Y \backslash 0$. 
As for the localized operators $A^{(3)}$, and $A^{(4)}$, we have

$$
\left\{\begin{aligned}
A_{(y, \eta)}^{(3)}(x, \xi)= & 0, \\
A_{(y, \eta)}^{(4)}(x, \xi)= & \left\langle\sigma_{m-1}(P)(y, \eta) x, x\right\rangle+\left\langle\sigma_{m-2}(Q)(y, \eta) x, \xi\right\rangle \\
& +\left\langle\sigma_{m-3}(R)(y, \eta) \xi, \xi\right\rangle+\sigma_{m-2}(T)(y, \eta) .
\end{aligned}\right.
$$

For the corresponding $d \times d$ (microlocal) system $\Lambda\left(y, D_{y}\right)$ of order $m-1$ in $Y$, from (90) we have

$$
\Lambda_{m-1}(y, \eta)=\operatorname{diag}\left(\lambda_{j}(y, \eta)\right)_{j=1, \ldots, d}, \quad \Lambda_{m-3 / 2}(y, \eta)=0,
$$

and

$$
\begin{aligned}
\Lambda_{m-2}^{\left(j^{\prime} j\right)}(y, \eta)= & \left(A_{(y, \eta)}^{(4)}\left(x, D_{x}\right) \psi_{j}(y, \eta ; .), \psi_{j^{\prime}}(y, \eta ; .)\right)_{L^{2}\left(\mathbb{R}^{v}\right)} \\
& +i \sum_{\ell=1}^{n-v} \frac{\partial \lambda_{j}}{\partial y_{\ell}}(y, \eta)\left(\frac{\partial \psi_{j}}{\partial \eta_{\ell}}(y, \eta ; .), \psi_{j^{\prime}}(y, \eta ; .)\right)_{L^{2}\left(\mathbb{R}^{v}\right)} \\
& \left.+\left(A_{(y, \eta)}^{(2)}\left(x, D_{x}\right)\left(\psi^{\prime}\right)_{j}\right)_{-1,1}(y, \eta ; .), \psi_{j^{\prime}}(y, \eta ; .)\right)_{L^{2}\left(\mathbb{R}^{v}\right)} \\
& +\frac{1}{i} \sum_{\ell=1}^{n-v}\left(\frac{\partial A_{(y, \eta)}^{(2)}}{\partial \eta_{\ell}}\left(x, D_{x}\right) \frac{\partial \psi_{j}}{\partial y_{\ell}}(y, \eta ; .), \psi_{j^{\prime}}(y, \eta ; .)\right)_{L^{2}\left(\mathbb{R}^{v}\right)}
\end{aligned}
$$

A crucial remark is that whenever $(y, \eta) \in \Sigma^{\prime}$, we have

$$
\begin{aligned}
\Lambda_{m-2}^{\left(j^{\prime} j\right)}(y, \eta) & =\left(A_{(y, \eta)}^{(4)}\left(x, D_{x}\right) \psi_{j}(y, \eta ; .), \psi_{j^{\prime}}(y, \eta ; .)\right)_{L^{2}\left(\mathbb{R}^{v}\right)} \\
+ & \frac{1}{i} \sum_{\ell=1}^{n-v}\left(\frac{\partial A_{(y, \eta)}^{(2)}}{\partial \eta_{\ell}}\left(x, D_{x}\right) \frac{\partial \psi_{j}}{\partial y_{\ell}}(y, \eta ; .), \psi_{j^{\prime}}(y, \eta ; .)\right)_{L^{2}\left(\mathbb{R}^{v}\right)}, \quad 1 \leq j^{\prime}, j \leq d .
\end{aligned}
$$

Fortunately enough, we know what the necessary and sufficient conditions for the hypoellipticity of $\Lambda\left(y, D_{y}\right)$ at $\left(y_{0}, \eta_{0}\right) \in \Sigma^{\prime}$ with loss of one derivative are, thanks to the Boutet-Grigis-Helffer Theorem in the system case (see [4] and also [2]). Namely, the conditions read as follows:

The matrix

$$
\Lambda_{m-2}\left(y_{0}, \eta_{0}\right)+\frac{i}{2}\left(\left\langle\partial_{y}, \partial_{\eta}\right\rangle \Lambda_{m-1}\right)\left(y_{0}, \eta_{0}\right)+\Delta_{\beta}\left(y_{0}, \eta_{0}\right)
$$

is invertible for all $\beta \in\left(\mathbb{Z}_{+}^{v^{\prime}}\right)^{d}$. 
This concludes the proof, once we define for $(y, \eta) \in \Sigma^{\prime}$

$$
\gamma(y, \eta):=\Lambda_{m-2}(y, \eta)+\frac{i}{2}\left(\left\langle\partial_{y}, \partial_{\eta}\right\rangle \Lambda_{m-1}\right)(y, \eta)
$$

REMARK 6.10. We have supposed that the eigenvalues $\lambda_{1}, \ldots, \lambda_{d}$ vanish on the same manifold $\Sigma^{\prime}$.

What happens (in case $d>1$ ) if the $\lambda_{j}$ vanish exactly to second order (for instance) on different $\Sigma_{j}^{\prime}\left(\right.$ with $\left.\left(y_{0}, \eta_{0}\right) \in \bigcap_{j=1}^{d} \Sigma_{j}^{\prime}\right)$ ?

To our knowledge, the conditions on $\Lambda_{m-2}$ and the geometry of the $\Sigma_{j}^{\prime}$ which ensure the hypoellipticity of $\Lambda\left(y, D_{y}\right)$ have not yet been found.

\subsection{Example}

We consider here a source of possible examples, all connected with the Heisenberg group (see also, for instance, Beals and Greiner [1]).

Consider in $\mathbb{R}_{t}^{v} \times \mathbb{R}_{s}^{v} \times \mathbb{R}_{y^{\prime}}$ the vector fields

$$
X_{j}=D_{t_{j}}+2 s_{j} D_{y^{\prime}}, \quad Y_{j}=D_{s_{j}}-2 t_{j} D_{y^{\prime}}, \quad j=1, \ldots, v,
$$

and recall that $X_{j}=X_{j}^{*}, Y_{j}=Y_{j}^{*}$ and

$$
\left[X_{j}, Y_{k}\right]=4 i \delta_{j k} D_{y^{\prime}}, \quad 1 \leq j, k \leq v .
$$

It is well known (see, e.g., [5]) that one can find a unitary Fourier integral operator $F$ of order zero such that

$$
X_{j} F=F D_{x_{j}}, \quad Y_{j} F=F x_{j} D_{y}, \quad j=1, \ldots, v,
$$

so that

$$
D_{y^{\prime}} F=F\left(-\frac{1}{4} D_{y}\right) .
$$

Now, with any given polynomial (with complex coefficients) $p=p\left(t, s, y^{\prime}\right)$, we can associate the left-invariant differential operator $p\left(X, Y, D_{y^{\prime}}\right)$. Hence,

$$
p\left(X, Y, D_{y^{\prime}}\right) F=F p\left(D_{x}, x D_{y},-\frac{1}{4} D_{y}\right),
$$

so that if $p$ satisfies suitable algebraic assumptions, the differential operator $p\left(D_{x}, x D_{y},-D_{y} / 4\right)$ fits the framework we developed here, allowing us to give hypoellipticity results for $p\left(X, Y, D_{y^{\prime}}\right)$.

For instance, just to mention one possibility, consider

$$
P=\sum_{j=1}^{v}\left(X_{j}^{2}+\mu_{j}^{2} Y_{j}^{2}\right)+\sum_{j=1}^{\nu}\left(\alpha_{j} X_{j}+\beta_{j} Y_{j}\right)+\gamma D_{y^{\prime}}+\delta
$$


with $\mu_{j}>0, \alpha_{j}, \beta_{j}, \gamma, \delta \in \mathbb{C}, j=1, \ldots, \nu$. Then $P F=F A$, where

$$
A=\sum_{j=1}^{\nu}\left(D_{x_{j}}^{2}+\mu_{j}^{2} x_{j}^{2} D_{y}^{2}\right)+\sum_{j=1}^{\nu}\left(\alpha_{j} D_{x_{j}}+\beta_{j} x_{j} D_{y}\right)-\frac{\gamma}{4} D_{y}+\delta .
$$

Then for the localized operator

$$
A_{(y, \eta)}^{(2)}=A_{\eta}^{(2)}=\sum_{j=1}^{\nu}\left(D_{x_{j}}^{2}+\mu_{j}^{2} x_{j}^{2} \eta^{2}\right)-\frac{\gamma}{4} \eta
$$

we have

$$
\operatorname{Spec}\left(A_{\eta}^{(2)}\right)=\left\{\sum_{j=1}^{v}\left(2 k_{j}+1\right) \mu_{j}|\eta|-\frac{\gamma}{4} \eta ; k \in \mathbb{Z}_{+}^{v}\right\} .
$$

Hence, $P$ is hypoelliptic with loss of one derivative if and only if

$$
\frac{\gamma}{4} \notin\left\{ \pm \sum_{j=1}^{\nu}\left(2 k_{j}+1\right) \mu_{j} ; k \in \mathbb{Z}_{+}^{v}\right\} .
$$

Consider now the case in which there are $d \geq 1$ multi-indices $k^{(1)}, \ldots, k^{(d)} \in \mathbb{Z}_{+}^{v}$ such that either

$$
\frac{\gamma}{4}=\sum_{j=1}^{\nu}\left(2 k_{j}^{(\ell)}+1\right) \mu_{j}, \quad \ell=1, \ldots, d,
$$

or

$$
\frac{\gamma}{4}=-\sum_{j=1}^{\nu}\left(2 k_{j}^{(\ell)}+1\right) \mu_{j}, \quad \ell=1, \ldots, d .
$$

For definiteness, suppose the former case holds.

We want to obtain the hypoellipticity of $A$ (and hence of $P$ ) with loss of two derivatives at every point $(x=0, y, \xi=0, \eta>0)$. A gauge for the trivial bundle $V=V_{1}=V_{2}$ is given by $\phi=\left(\phi_{k^{(1)}}, \ldots, \phi_{k^{(d)}}\right)$, with

$$
\phi_{k^{(\ell)}}=\phi_{k^{(\ell)}}(\eta ; x)=\prod_{j=1}^{v}\left(\mu_{j}|\eta|\right)^{1 / 4} h_{k_{j}^{(\ell)}}\left(\sqrt{\mu_{j}|\eta|} x_{j}\right), \quad \ell=1, \ldots, d .
$$

Now

$$
\left\{\begin{array}{l}
A_{\eta}^{(3)}=\sum_{j=1}^{\nu}\left(\alpha_{j} D_{x_{j}}+\beta_{j} x_{j} \eta\right) \\
A_{\eta}^{(4)}=\delta
\end{array}\right.
$$


hence, for the corresponding $\Lambda\left(y, D_{y}\right)$, we have (for $\eta>0$ )

$$
\left\{\begin{array}{l}
\Lambda_{1}(y, \eta)=0, \\
\Lambda_{1 / 2}(y, \eta)=0, \\
\Lambda_{0}(y, \eta)=\delta I_{d}-\left(\left(E_{\eta}^{(-2)} A_{\eta}^{(3)} \phi_{k^{\left(\ell^{\prime}\right)}}(\eta ; .),\left(A_{\eta}^{(3)}\right)^{*} \phi_{k^{(\ell)}}(\eta ; .)\right)_{L^{2}\left(\mathbb{R}^{v}\right)}\right)_{1 \leq \ell, \ell^{\prime} \leq d} .
\end{array}\right.
$$

By computations similar to those carried out in Example 6.1, we get

$$
\begin{aligned}
& -\left(E_{\eta}^{(-2)} A_{\eta}^{(3)} \phi_{k^{\left(\ell^{\prime}\right)}}(\eta ; .),\left(A_{\eta}^{(3)}\right)^{*} \phi_{k^{(\ell)}}(\eta ; .)\right)_{L^{2}\left(\mathbb{R}^{v}\right)} \\
& =-\sum_{j, j^{\prime}=1}^{v} \sum_{k \neq k^{(1)}, \ldots, k^{(d)}} \frac{1}{\lambda_{k}(\eta)}\left[\left(m_{j} \sqrt{2\left(k_{j}^{\left(\ell^{\prime}\right)}+1\right) \mu_{j}|\eta|}-\frac{r_{j}}{2} \sqrt{2\left(k_{j}^{\left(\ell^{\prime}\right)}+1\right)}\right) \delta_{k^{\left(\ell^{\prime}\right)}+e_{j}, k}\right. \\
& \left.-\frac{r_{j}}{2} \sqrt{2 k_{j}^{\left(\ell^{\prime}\right)}} \delta_{k^{\left(\ell^{\prime}\right)}-e_{j}, k}\right]\left[\left(m_{j^{\prime}} \sqrt{2 k_{j^{\prime}}^{(\ell)} \mu_{j^{\prime}}|\eta|}-\frac{r_{j^{\prime}}}{2} \sqrt{2 k_{j^{\prime}}^{(\ell)}}\right) \delta_{k^{(\ell)}-e_{j^{\prime}}, k}\right. \\
& \left.-\frac{r_{j^{\prime}}}{2} \sqrt{2\left(k_{j^{\prime}}^{(\ell)}+1\right)} \delta_{k^{(\ell)}+e_{j^{\prime}}, k}\right],
\end{aligned}
$$

where

$$
m_{j}=-i \alpha_{j}, \quad r_{j}=-i \alpha_{j} \sqrt{\mu_{j}|\eta|}+\beta_{j} \sqrt{\frac{|\eta|}{\mu_{j}}}
$$

and

$$
\lambda_{k}(\eta)=2|\eta| \sum_{j=1}^{\nu}\left(k_{j}-k_{j}^{(\ell)}\right) \mu_{j}
$$

which is independent of $\ell=1, \ldots, d$.

Note that $\Lambda_{0}$ is a $d \times d$ complex matrix, which is independent of $y$ and $\eta>0$. We get that $P$ is hypoelliptic with loss of two derivatives if and only if $\Lambda_{0}$ is invertible. Note also that when $\alpha=\beta=0$, the previous condition reduces to $\delta \neq 0$, which recaptures, in the $C^{\infty}$-hypoellipticity, the well-known example by Stein [20].

\subsection{Example: iteration of the machinery}

In this last example, we revisit Example 6.2 to show a very simple instance in which the whole machinery can be iterated by micro-microlocalizing.

Consider in $\mathbb{R}_{x} \times \mathbb{R}_{y}^{n-1}(n \geq 3)$ the operator

$$
A=\left(D_{x}^{2}+\mu^{2} x^{2}\left|D_{y}\right|^{2}\right)^{2}+\left(1-\mu^{2}\right) D_{y_{1}}^{2}+\left(\sigma^{2} y_{1}^{2}-\mu^{2}\right)\left|D_{y^{\prime}}\right|^{2}+a D_{x}^{2}+\gamma,
$$

where $a, \gamma \in \mathbb{C}$ and $\mu, \sigma \in \mathbb{R}_{+}$. We shall prove the following (rather surprising) result. 
Proposition 6.11. For the hypoellipticity of $A$, we have:

(i) $A$ is hypoelliptic with loss of two derivatives at every point $\left(x=0, y_{1}\right.$, $\left.y^{\prime}, \xi=0, \eta_{1}, \eta^{\prime}\right)$ for which $\left(y_{1}, \eta_{1}\right) \neq(0,0)$.

(ii) At every point of the submanifold $\Sigma^{\prime} \subset \Sigma, \Sigma^{\prime}=\left\{\left(x=0, y_{1}=0, y^{\prime}\right.\right.$, $\left.\left.\xi=0, \eta_{1}=0, \eta^{\prime}\right) ; y^{\prime}, \eta^{\prime} \in \mathbb{R}^{n-2}, \eta^{\prime} \neq 0\right\}$, A can be hypoelliptic only with a loss of derivatives $\geq 3$ and, in fact, is hypoelliptic with loss of three derivatives if and only if

$$
a \neq-\frac{2 \sigma}{\mu}(2 j+1), \quad \text { for all } j \in \mathbb{Z}_{+} .
$$

(iii) If $a=-(2 \sigma / \mu)(2 k+1)$ for some $k \in \mathbb{Z}_{+}$, A can be hypoelliptic at the points of $\Sigma^{\prime}$ only with a loss of derivatives $\geq 4$, and, in fact, is hypoelliptic with loss of four derivatives if and only if

$$
\gamma \neq \frac{(2 k+1)^{2} \sigma^{2}}{4}\left(1+\frac{1}{3 \mu^{2}}\right) .
$$

(iv) If

$$
a=-\frac{2 \sigma}{\mu}(2 k+1) \quad \text { and } \quad \gamma=\frac{(2 k+1)^{2} \sigma^{2}}{4}\left(1+\frac{1}{3 \mu^{2}}\right),
$$

for some $k \in \mathbb{Z}_{+}$, A can be hypoelliptic at the points of $\Sigma^{\prime}$ only with a loss of derivatives $\geq 5$ and, in fact, is hypoelliptic with loss of five derivatives if and only if

$$
\mu^{4} \neq \frac{2}{9} \frac{(2 k+1)^{2}}{k^{2}+k+2}
$$

Proof. From Example 6.2, we recall what the localized operators $A_{(y, \eta)}^{(j)}, j \geq 4$, are. Precisely one has

$$
\left\{\begin{array}{l}
A_{(y, \eta)}^{(4)}=\left(D_{x}^{2}+\mu^{2} x^{2}|\eta|^{2}\right)^{2}+\left(1-\mu^{2}\right) \eta_{1}^{2}+\left(\sigma^{2} y_{1}^{2}-\mu^{2}\right)\left|\eta^{\prime}\right|^{2} \\
A_{(y, \eta)}^{(5)}=0, \\
A_{(y, \eta)}^{(6)}=a D_{x}^{2} \\
A_{(y, \eta)}^{(7)}=0, \\
A_{(y, \eta)}^{(8)}=\gamma, \\
A_{(y, \eta)}^{(j)}=0, \quad \text { for all } j \geq 9 .
\end{array}\right.
$$


Moreover, one has that $A_{(y, \eta)}^{(4)}=\left(A_{(y, \eta)}^{(4)}\right)^{*}$ has a spectrum made of the eigenvalues

$$
\lambda_{k}(y, \eta)=\left(\left(4 k^{2}+4 k\right) \mu^{2}+1\right) \eta_{1}^{2}+\left(\left(4 k^{2}+4 k\right) \mu^{2}+\sigma^{2} y_{1}^{2}\right)\left|\eta^{\prime}\right|^{2}, \quad k \in \mathbb{Z}_{+},
$$

with corresponding normalized eigenfunctions

$$
\phi_{k}(\eta ; x)=(\mu|\eta|)^{1 / 4} h_{k}(\sqrt{\mu|\eta|} x), \quad k \in \mathbb{Z}_{+},
$$

with $h_{k}$ given in (95).

When $\rho \notin \Sigma^{\prime}$, every $\lambda_{k}>0$, whence the Boutet-Grigis-Helffer Theorem 1.4 gives (i) in the statement.

Suppose now that $\rho \in \Sigma^{\prime}$. In this case, the lowest eigenvalue $\lambda_{0}$ vanishes exactly to second order at $\rho$. According to our Theorem 5.1, we have to construct the scalar operator $\Lambda\left(y, D_{y}\right) \in \operatorname{OPS}_{\mathrm{cl}}^{2}\left(\mathbb{R}^{n-1}\right)$. In order to prove the proposition, we have to compute the terms $\Lambda_{2-j / 2}(y, \eta), j=0,1, \ldots, 6$, in the symbol of $\Lambda\left(y, D_{y}\right)$. We repeatedly use formulas (83) and (84) (with $\phi_{-p / 2}=\phi_{-p / 2}^{\prime}=0$ for every $p \geq 1$, and $\left.\phi_{0}^{\prime}=\phi_{0}=\psi_{0}=\psi_{0}^{\prime}\right)$. By an elementary and rather messy computation, one gets

$$
\begin{gathered}
\left\{\begin{array}{l}
\Lambda_{2}(y, \eta)=\lambda_{0}(y, \eta)=\eta_{1}^{2}+\sigma^{2} y_{1}^{2}\left|\eta^{\prime}\right|^{2}, \\
\psi_{0}^{\prime}=\phi_{0}=(\mu|\eta|)^{1 / 4} h_{0}(\sqrt{\mu|\eta|} x),
\end{array}\right. \\
\left\{\begin{array}{l}
\Lambda_{3 / 2}(y, \eta)=0, \\
\psi_{-1 / 2}^{\prime}=0,
\end{array}\right. \\
\left\{\begin{array}{l}
\Lambda_{1}(y, \eta)=\frac{1}{2} a \mu|\eta|, \\
\psi_{-1}^{\prime}=\frac{\sqrt{2}}{2} \frac{F(y, \eta)}{\lambda_{2}(y, \eta)} \phi_{2},
\end{array}\right.
\end{gathered}
$$

where

$$
\begin{gathered}
F(y, \eta):=\frac{a \mu|\eta|^{3}+i \sigma^{2} y_{1} \eta_{1}\left|\eta^{\prime}\right|^{2}}{|\eta|^{2}}, \\
\left\{\begin{array}{l}
\Lambda_{1 / 2}(y, \eta)=0 \\
\psi_{-3 / 2}^{\prime}=0,
\end{array}\right. \\
\left\{\begin{array}{l}
\Lambda_{0}(y, \eta)=\frac{1}{4 i} \frac{\eta_{1}}{|\eta|^{2}} \frac{\partial}{\partial y_{1}}\left(\frac{F}{\lambda_{2}}\right) \Lambda_{2}-\frac{\sigma^{2}}{8} \frac{\eta_{1}^{2}\left|\eta^{\prime}\right|^{2}}{|\eta|^{4}}+\frac{6 \mu^{2}}{i} \eta_{1} \frac{\partial}{\partial y_{1}}\left(\frac{F}{\lambda_{2}}\right)-\frac{a \mu}{2} \frac{F}{\lambda_{2}}|\eta|+\gamma, \\
\psi_{-2}^{\prime}=\frac{1}{4 i} \frac{\eta_{1}}{|\eta|^{2}} \frac{\partial}{\partial y_{1}}\left(\frac{F}{\lambda_{2}}\right) \phi_{0}-\sqrt{2} H \phi_{2}-\sqrt{6} G \phi_{4},
\end{array}\right.
\end{gathered}
$$


where

$$
\begin{gathered}
H(y, \eta):=\frac{1}{\lambda_{2}(y, \eta)}\left[\frac{\sigma^{2}\left|\eta^{\prime}\right|^{2}}{4|\eta|^{2}}\left(\eta_{1}^{2}-\left|\eta^{\prime}\right|^{2}\right)-i\left(1+24 \mu^{2}\right) \eta_{1} \frac{\partial}{\partial y_{1}}\left(\frac{F}{\lambda_{2}}\right)+\frac{5}{4} a \mu|\eta| \frac{F}{\lambda_{2}}\right], \\
G(y, \eta):=\frac{1}{\lambda_{4}(y, \eta)}\left[\frac{\sigma^{2}}{8|\eta|^{4}} \eta_{1}^{2}\left|\eta^{\prime}\right|^{2}-14 i \mu^{2} \eta_{1} \frac{\partial}{\partial y_{1}}\left(\frac{F}{\lambda_{2}}\right)-\frac{a \mu}{2}|\eta| \frac{F}{\lambda_{2}}\right], \\
\left\{\begin{array}{l}
\Lambda_{-1 / 2}(y, \eta)=0, \\
\psi_{-5 / 2}^{\prime}=0,
\end{array}\right.
\end{gathered}
$$

and

$$
\begin{aligned}
\Lambda_{-1}(y, \eta)= & {\left[\frac{1}{8} \frac{\eta_{1}^{2}-\left|\eta^{\prime}\right|^{2}}{|\eta|^{4}} \frac{\partial^{2}}{\partial y_{1}^{2}}\left(\frac{F}{\lambda_{2}}\right)-\frac{1}{2 i} \frac{\eta_{1}}{|\eta|^{2}} \frac{\partial H}{\partial y_{1}}\right] \Lambda_{2}(y, \eta) } \\
& -3\left(\mu^{2}+2 \frac{\eta_{1}^{2}}{|\eta|^{2}}\right) \frac{\partial^{2}}{\partial y_{1}^{2}}\left(\frac{F}{\lambda_{2}}\right)-\frac{1}{2} \frac{\eta_{1}^{2}}{|\eta|^{2}} \frac{\partial^{2}}{\partial y_{1}^{2}}\left(\frac{F}{\lambda_{2}}\right) \\
& -\frac{12}{i} \mu^{2} \eta_{1} \frac{\partial H}{\partial y_{1}}+\frac{a \mu}{8 i|\eta|} \eta_{1} \frac{\partial}{\partial y_{1}}\left(\frac{F}{\lambda_{2}}\right)+a \mu|\eta| H .
\end{aligned}
$$

In the computations, it is convenient to use the formulas

$$
\left\{\begin{aligned}
x^{2} \phi_{k}= & \frac{1}{\mu|\eta|}\left[\frac{\sqrt{k(k-1)}}{2} \phi_{k-2}+\frac{2 k+1}{2} \phi_{k}+\frac{\sqrt{(k+1)(k+2)}}{2} \phi_{k+2}\right] \\
D_{x}^{2} \phi_{k}= & -\mu|\eta|\left[\frac{\sqrt{k(k-1)}}{2} \phi_{k-2}-\frac{2 k+1}{2} \phi_{k}+\frac{\sqrt{(k+1)(k+2)}}{2} \phi_{k+2}\right] \\
\frac{\partial \phi_{k}}{\partial \eta_{1}}= & \frac{\eta_{1}}{4|\eta|^{2}}\left[\sqrt{k(k-1)} \phi_{k-2}-\sqrt{(k+1)(k+2)} \phi_{k+2}\right] \\
\frac{\partial^{2} \phi_{k}}{\partial \eta_{1}^{2}}= & \frac{\eta_{1}^{2}}{16|\eta|^{4}} \sqrt{(k-3)(k-2)(k-1) k} \phi_{k-4}-\frac{\eta_{1}^{2}-\left|\eta^{\prime}\right|^{2}}{4|\eta|^{4}} \sqrt{k(k-1)} \phi_{k-2} \\
& -\frac{\eta_{1}^{2}}{8|\eta|^{4}}\left(k^{2}+k+1\right) \phi_{k}+\frac{\eta_{1}^{2}-\left|\eta^{\prime}\right|^{2}}{4|\eta|^{4}} \sqrt{(k+1)(k+2)} \phi_{k+2} \\
& +\frac{\eta_{1}^{2}}{16|\eta|^{4}} \sqrt{(k+1)(k+2)(k+3)(k+4)} \phi_{k+4} .
\end{aligned}\right.
$$

Now observe that $\Lambda\left(y, D_{y}\right)$ has double characteristics and is transversally elliptic with respect to $\Sigma^{\prime}$. We have, hence, to compute the localized operators $\Lambda_{\left(y^{\prime}, \eta^{\prime}\right)}^{(j)}, j \geq 2$ 
and, in fact, for $j$ up to 6 , in order to prove the proposition. Once more, a computation gives

$$
\begin{aligned}
\Lambda_{\left(y^{\prime}, \eta^{\prime}\right)}^{(2)}= & D_{y_{1}}^{2}+\sigma^{2} y_{1}^{2}\left|\eta^{\prime}\right|^{2}+\frac{a \mu}{2}\left|\eta^{\prime}\right|, \\
\Lambda_{\left(y^{\prime}, \eta^{\prime}\right)}^{(3)}= & 0, \\
\Lambda_{\left(y^{\prime}, \eta^{\prime}\right)}^{(4)}= & \frac{a \mu}{4\left|\eta^{\prime}\right|} D_{y_{1}}^{2}+\gamma-\frac{a^{2}}{48}, \\
\Lambda_{\left(y^{\prime}, \eta^{\prime}\right)}^{(5)}= & 0, \\
\Lambda_{\left(y^{\prime}, \eta^{\prime}\right)}^{(6)}= & -\frac{a \mu}{16\left|\eta^{\prime}\right|^{3}} D_{y_{1}}^{4}+\frac{1}{8\left|\eta^{\prime}\right|^{2}}\left(\sigma^{2}+\frac{a^{2}}{6 \cdot 24 \mu^{2}}\right) D_{y_{1}}^{2} \\
& +\frac{a^{2} \sigma^{2}}{2 \cdot 24^{2} \mu^{2}} y_{1}^{2}+\frac{5 a^{3}}{4 \cdot 24^{2} \mu\left|\eta^{\prime}\right|} .
\end{aligned}
$$

Now, the eigenvalues of $\Lambda_{\left(y^{\prime}, \eta^{\prime}\right)}^{(2)}$ are

$$
\omega_{j}\left(y^{\prime}, \eta^{\prime}\right)=\left(\frac{a \mu}{2}+(2 j+1) \sigma\right)\left|\eta^{\prime}\right|, \quad j \in \mathbb{Z}_{+},
$$

with corresponding normalized eigenfunctions

$$
\theta_{j}\left(\eta^{\prime} ; y_{1}\right)=\left(\sigma\left|\eta^{\prime}\right|\right)^{1 / 4} h_{j}\left(\sqrt{\sigma\left|\eta^{\prime}\right|} y_{1}\right), \quad j \in \mathbb{Z}_{+} .
$$

By the Boutet-Grigis-Helffer Theorem, $\Lambda\left(y, D_{y}\right)$ is hypoelliptic with loss of one derivative at $\rho \in \Sigma^{\prime}$ if and only if $\omega_{j}(\rho) \neq 0$ for all $j \in \mathbb{Z}_{+}$, that is to say if and only if

$$
a \neq-\frac{2 \sigma}{\mu}(2 j+1), \quad \text { for all } j \in \mathbb{Z}_{+}
$$

This and Theorem 5.1 give point (ii) of the proposition.

Suppose now that $a=-(2 \sigma / \mu)(2 k+1)$ for some $k \in \mathbb{Z}_{+}$. To apply our machinery once more, we have to compute the associated operator $\tilde{\Lambda}\left(y^{\prime}, D_{y^{\prime}}\right) \in$ $\operatorname{OPS}_{\mathrm{cl}}^{1}\left(\mathbb{R}^{n-2}\right)$ and, more precisely, the terms $\tilde{\Lambda}_{1}\left(y^{\prime}, \eta^{\prime}\right), \ldots, \tilde{\Lambda}_{-1}\left(y^{\prime}, \eta^{\prime}\right)$ of its symbol. Once again, we use formulas (83) and (84) (with $\phi_{0}=\phi_{0}^{\prime}=\psi_{0}^{\prime}=\psi_{0}^{\prime}=\theta_{k}$ and 
$\phi_{-p / 2}=\phi_{-p / 2}^{\prime}=0$ for all $\left.p \geq 1\right)$ and obtain

$$
\begin{aligned}
& \left\{\begin{array}{l}
\tilde{\Lambda}_{1}\left(y^{\prime}, \eta^{\prime}\right)=0, \\
\psi_{0}^{\prime}=\theta_{k}
\end{array}\right. \\
& \left\{\begin{array}{l}
\tilde{\Lambda}_{1 / 2}\left(y^{\prime}, \eta^{\prime}\right)=0, \\
\psi_{-1 / 2}^{\prime}=0,
\end{array}\right. \\
& \left\{\begin{array}{l}
\tilde{\Lambda}_{0}\left(y^{\prime}, \eta^{\prime}\right)=\gamma-\frac{(2 k+1)^{2} \sigma^{2}}{4}\left(1+\frac{1}{3 \mu^{2}}\right), \\
\psi_{-1}^{\prime}=-\frac{\sigma}{16} \frac{2 k+1}{\left|\eta^{\prime}\right|}\left(\sqrt{(k+1)(k+2)} \theta_{k+2}-\sqrt{k(k-1)} \theta_{k-2}\right),
\end{array}\right. \\
& \left\{\begin{array}{l}
\tilde{\Lambda}_{-1 / 2}\left(y^{\prime}, \eta^{\prime}\right)=0 \\
\psi_{-3 / 2}^{\prime}=0,
\end{array}\right. \\
& \tilde{\Lambda}_{-1}\left(y^{\prime}, \eta^{\prime}\right)=\frac{\sigma^{3}(2 k+1)}{8\left|\eta^{\prime}\right|}\left(\frac{k^{2}+k+2}{2}-\frac{1}{9 \mu^{4}}(2 k+1)^{2}\right) .
\end{aligned}
$$

In the computations, it is convenient to use an analogue of formulas (140) for $\theta_{k}$.

As a consequence, $\Lambda\left(y, D_{y}\right)$ is hypoelliptic at $\rho \in \Sigma^{\prime}$ with loss of two derivatives if and only if $\tilde{\Lambda}_{0}(\rho) \neq 0$, that is to say if and only if

$$
\gamma \neq \frac{(2 k+1)^{2} \sigma^{2}}{4}\left(1+\frac{1}{3 \mu^{2}}\right)
$$

which yields point (iii) in the statement.

Finally, when

$$
\gamma=\frac{(2 k+1)^{2} \sigma^{2}}{4}\left(1+\frac{1}{3 \mu^{2}}\right)
$$

we have that $\tilde{\Lambda}\left(y^{\prime}, D_{y^{\prime}}\right)$ is hypoelliptic with loss of two derivatives if and only if $\tilde{\Lambda}_{-1} \neq 0$, so that by our Theorem $5.1 \Lambda\left(y, D_{y}\right)$ is hypoelliptic at $\rho \in \Sigma^{\prime}$ with loss of three derivatives if and only if

$$
\mu^{4} \neq \frac{2}{9} \frac{(2 k+1)^{2}}{k^{2}+k+2}
$$

This proves point (iv) and concludes the proof of the proposition.

REMARK 6.12. If

$$
\mu^{4}=\frac{2}{9} \frac{(2 k+1)^{2}}{k^{2}+k+2}
$$


to detect the hypoellipticity of $\tilde{\Lambda}\left(y^{\prime}, D_{y^{\prime}}\right)$ (and, hence, of $\Lambda\left(y, D_{y}\right)$ and, hence, of $\left.A\right)$, one should compute the terms $\tilde{\Lambda}_{1-j / 2}, j \geq 5$, but this requires knowledge of the terms $\Lambda_{2-j / 2}, j \geq 7$, a computation that we decided not to carry out!

\section{Conclusive remarks: invariance?}

We finally comment on the lack of invariance in our approach.

From the very beginning, we have supposed that the characteristic manifold $\Sigma$ of $A$ be flat. Now, suppose $\Sigma$ is a general symplectic submanifold of $T^{*} \mathbb{R}^{n} \backslash 0$ of codimension $2 v$, conditions (1) and (2) hold and we ask for the hypoellipticity of $A$ at some point $\rho_{0} \in \Sigma$. Again, one has the Boutet-Grigis-Helffer Theorem 1.4, for the localized operator $A_{\rho}, \rho \in \Sigma$, is intrinsically defined in the following way (see [6] and [15]).

One takes the Weyl symbol of $A$

$$
\sigma_{\text {Weyl }}(A):=e^{i\left\langle\partial_{z}, \partial_{\zeta}\right\rangle / 2} \sigma(A) \sim \sum_{j \geq 0} q_{m-j / 2}
$$

and next defines the polynomial map

$$
q_{\rho}: T_{\rho} \Sigma^{\sigma} \longrightarrow \mathbb{C}, \quad \rho \in \Sigma,
$$

by

$$
q_{\rho}(v)=\sum_{j=0}^{k} \frac{1}{(k-j) !}\left(V^{k-j} q_{m-j / 2}\right)(\rho),
$$

where $v \in T_{\rho} \Sigma^{\sigma}$ and $V$ is any smooth vector field on $T^{*} \mathbb{R}^{n}$ with $V(\rho)=v$ (the vanishing conditions on the $q_{m-j / 2}$ ensure that the definition of $q_{\rho}(v)$ is independent of the extension $V$ of $v$ ). Now take any linear symplectomorphism

$$
f: T^{*} \mathbb{R}^{v} \simeq \mathbb{R}_{x}^{v} \times \mathbb{R}_{\xi}^{v} \longrightarrow T_{\rho} \Sigma^{\sigma} .
$$

Put $q_{\rho, f}(x, \xi):=q_{\rho}(f(x, \xi))$ and

$$
A_{\rho, f}:=\mathrm{Op}^{\mathrm{w}}\left(q_{\rho, f}\right)\left(x, D_{x}\right)
$$

and observe that (by Theorem 18.5.9 of Hörmander [10, Vol. III]) if $f_{1}$ and $f_{2}$ are two such linear symplectomorphisms, $A_{\rho, f_{1}}$ and $A_{\rho, f_{2}}$ are unitarily equivalent then, so that the spectral properties of the $A_{\rho, f}$ are independent of the symplectomorphism $f$.

Note that if we perform the previous construction when $\Sigma$ is flat, we get that $\mathrm{Op}^{\mathrm{w}}\left(q_{\rho, \text { id }}\right)\left(x, D_{x}\right)$ coincides with the localized operator $A_{\rho}^{(k)}$, that we have used throughout the paper. Here id is the canonical identification of $T^{*} \mathbb{R}^{\nu}$ with $T_{\rho} \Sigma^{\sigma}$.

Theorem 1.4 now reads: 
A is hypoelliptic at $\rho_{0} \in \Sigma$ with loss of $k / 2$ derivatives if and only if $\operatorname{Ker}\left(A_{\rho_{0}, f}\right)=\{0\}$ for one and, hence, for all $f$ as above.

Thus, the problem is:

What can one say on the hypoellipticity of $A$ at $\rho_{0} \in \Sigma$ when the injectivity condition is not satisfied?

Our recipe goes as follows:

- $\quad$ take a homogeneous canonical transformation $\chi$ that brings $\Sigma$ into the flat case $x=\xi=0$;

- $\quad$ consider the $\psi$ do $\tilde{A}$ obtained by conjugating $A$ with an elliptic Fourier integral operator associated with $\chi$;

- $\quad$ 'compute' $\Lambda\left(y, D_{y}\right)$.

It is important to observe that one has

$$
\tilde{A}_{\chi\left(\rho_{0}\right)}^{(k)}=A_{\rho_{0}, f},
$$

with $f(x, \xi)=$ the projection onto $T_{\rho} \Sigma^{\sigma}$ of $\chi^{\prime}\left(\rho_{0}\right)^{-1}\left[\begin{array}{l}x \\ 0 \\ \xi \\ 0\end{array}\right]$.

One of the crucial problems is, therefore, the following:

Is there an object (on $\Sigma$ ) that corresponds, via $\chi$, to the higher-order localized operator $\tilde{A}_{\chi\left(\rho_{0}\right)}^{(k+j)}$ ?

We do not have an answer. For this reason, we had to work in the flat case. An answer to this problem would lead to an 'invariant' interpretation of the conditions obtained in the previously treated examples.

Acknowledgement. This paper was completed while the second author was Ryuudou Visiting Professor at the Tokyo Institute of Technology. He wishes to thank the TIT for the excellent hospitality during his stay.

\section{REFERENCES}

[1] R. Beals and P. C. Greiner. Calculus on Heisenberg Manifolds (Annals of Mathematics Studies, 119). Princeton University Press, Princeton, 1988.

[2] L. Boutet de Monvel and F. Treves. On a class of systems of pseudodifferential equations with double characteristics. Comm. Pure Appl. Math. 27 (1974), 59-89.

[3] L. Boutet de Monvel. Hypoelliptic operators with double characteristics and related pseudodifferential operators. Comm. Pure Appl. Math. 27 (1974), 585-639. 
[4] L. Boutet de Monvel, A. Grigis and B. Helffer. Paramétrixes d'opérateurs pseudo-différentiels à caractéristiques multiples. Astérisque 34-35 (1976), 93-121.

[5] A. Grigis and L. P. Rothschild. A criterion for analytic hypoellipticity of a class of differential operators with polynomial coefficients. Ann. Math. 118 (1983), 443-460.

[6] B. Helffer. Invariants associés à une classe d'opérateurs pseudodifférentiels et applications à l'hypoellipticité. Ann. Inst. Fourier (Grenoble) 26(2) (1976), 55-70.

[7] B. Helffer. Sur l'hypoellipticité des opérateurs pseudodifferentiels à caractéristiques multiples (perte de 3/2 dérivées). Bull. Soc. Math. France Suppl. Mém. 51-52 (1977), 13-61.

[8] B. Helffer. Théorie spectrale pour des opérateurs globalement elliptiques Astérisque 112 (1984).

[9] L. Hörmander. A class of hypoelliptic pseudodifferential operators with double characteristics. Math. Ann. 217(2) (1975), 165-188.

[10] L. Hörmander. The Analysis of Linear Partial Differential Operators, Vol. I-IV. Springer, Berlin, $1983 / 85$.

[11] V. Ivrii. Wave front of solutions of certain pseudodifferential equations. Trans. Moscow Math. Soc. 1 (1981), 49-86

[12] K. H. Kwon. Concatenations applied to analytic hypoellipticity of operators with double characteristics. Trans. Amer. Math. Soc. 283(2) (1984), 753-763.

[13] G. Métivier. Analytic hypoellipticity for operators with multiple characteristics. Comm. Partial Differential Equations 6 (1981), 1-90.

[14] A. Mohamed. Etude spectrale d'opérateurs hypoelliptiques à caractéristiques multiples, II. Comm. Partial Differential Equations 8 (1983), 247-316.

[15] C. Parenti and A. Parmeggiani. Some remarks on almost-positivity of $\psi$ do's. Boll. Unione Mat. Ital. Sez. B Artic. Ric. Mat. 8(1) (1998), 187-215.

[16] C. Parenti and A. Parmeggiani. A generalization of Hörmander's inequality I. Comm. Partial Differential Equations 25(3-4) (2000), 457-506.

[17] C. Parenti and A. Parmeggiani. Lower bounds for systems with double characteristics. J. Anal. Math. 86 (2002), 49-91.

[18] M. Shubin. Pseudodifferential Operators and Spectral Theory. Springer, Berlin, 1987.

[19] J. Sjöstrand. Parametrices for pseudodifferential operators with multiple characteristics. Ark. Mat. 12 (1974), 85-130.

[20] E. M. Stein. An example on the Heisenberg group related to the Levy operator. Invent. Math. 69 (1982), 209-216.

[21] F. Treves. Introduction to Pseudodifferential and Fourier Integral Operators, I. Plenum Press, New York, 1980. 
Cesare Parenti

Department of Computer Science

University of Bologna

Via Mura Anteo Zamboni 7

40127 Bologna

Italy

(E-mail:parenti@cs.unibo.it)

Alberto Parmeggiani

School of Science

Tokyo Institute of Technology

2-12-1 Oh-okayama, Meguro-ku,

Tokyo, 152-8551

Japan

Current address:

Department of Mathematics

University of Bologna

Piazza di Porta S. Donato 5

40127 Bologna

Italy

(E-mail: parmeggi@dm.unibo.it) 